\title{
Preoperative fluid management of the older adult patient with hip fracture
}

\author{
By \\ Victoria Carolyn Ward
}

\begin{abstract}
A thesis
submitted to Victoria University of Wellington

in partial fulfillment of the requirements for the degree of Master of Clinical Research
\end{abstract}

Victoria University of Wellington 
PREOPERATIVE FLUIDS 


\section{Abstract}

\section{Background}

While recommendations about the preoperative fluid management of the older adult hip fracture patient have been produced the evidence relating to postoperative outcomes is scant.

\section{Aim}

The aim of this study is, therefore, to explore the relationships between preoperative fluid management (PFM) - defined as timing to start of fluids (oral or intravenous) after admission, preoperative oral fluid rate, and timing of last preoperative oral fluids - and postoperative outcomes - defined as change in renal function (creatinine and GFR), new reported postoperative confusion, timing of discontinuation of postoperative intravenous fluids (IV), and length of stay (LOS).

\section{Method}

This observational study looked at 100 consecutive older adult patients admitted to a tertiary New Zealand hospital with traumatic hip fracture between March and September, 2012. Data was gathered regarding cohort demographics and in hospital events, including surgical details, alongside PFM and postoperative outcomes.

Descriptive statistics, linear regression, independent t-tests, tests of equality, and multiple logistic regression were utilised to ascertain relationships between variables.

\section{Results}

Characteristics of the perioperative journey of 100 consecutive patients, with mean age of $85.2 \mathrm{yr}$ (SD 6.6) and predominantly female (70\%), presenting to CCDHB with a fragility hip fracture were itemised. High rates of co-morbidities were observed in this group, with $92 \%$ of patients having cardiac, pulmonary, vascular or renal comorbidities, but with a mortality rate $(2 \%)$ much lower than the literature would suggest might be 
expected. There was no substantiated statistically significant relationships observed between preoperative fluid management in this cohort and postoperative outcomes, but this may reflect limited power in this study.

\section{Conclusion}

These results provide useful data for planning services with regards management of the older adult hip fracture patient at CCDHB. In addition, the study has highlighted a number of clinical guidelines that might be more effectively promoted. 


\section{Acknowledgments}

I wish to acknowledge all the wonderful people, friends and relatives, who have inspired, assisted and supported me through my master's journey. They are too many to name here but they know who they are.

I do, however, wish to name a couple of key people in this endeavor, namely Professor Geoff Horne who was a key supporter and instigator of my thesis project, and Professor Elaine Dennison, my forever busy and enthusiastic supervisor.

And finally I dedicate this thesis to my children, Clare and Peter, mother Felicity, and my late father Peter. 


\section{Table of contents}

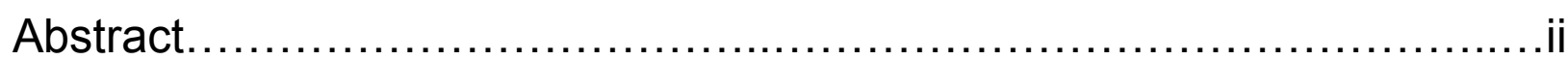

Acknowledgements.....................

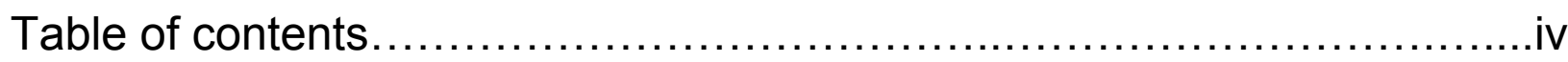

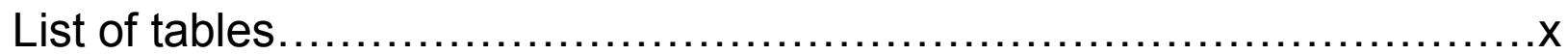

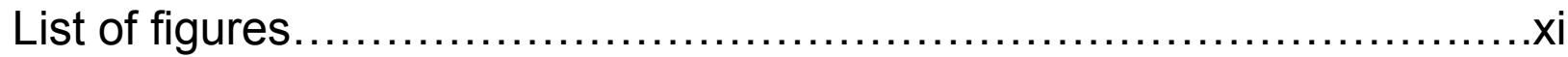

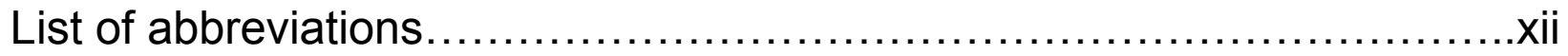

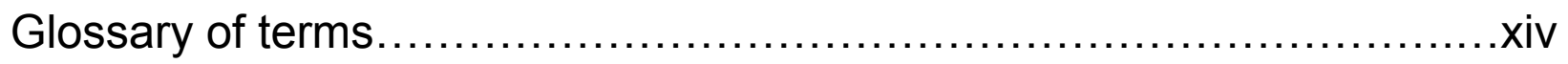

Chapter 1: Introduction......................................

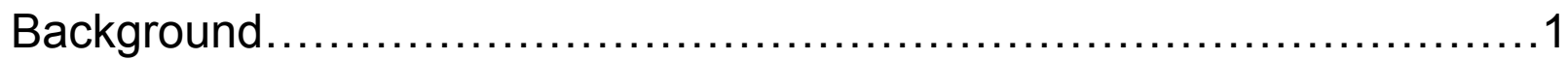

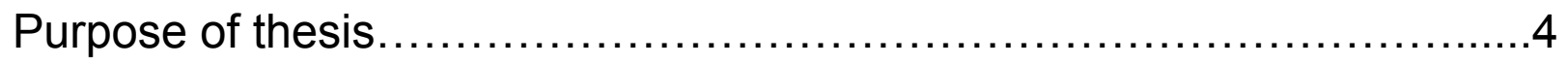

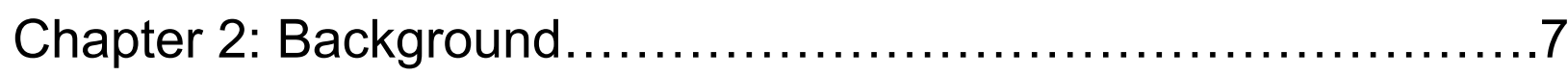

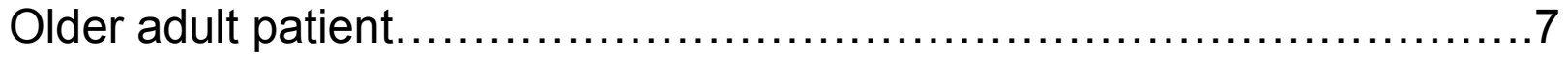

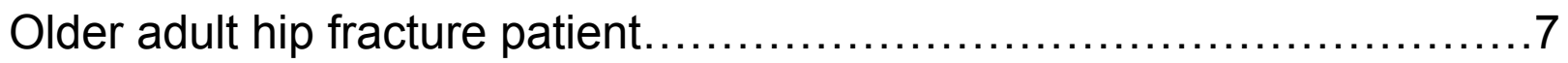

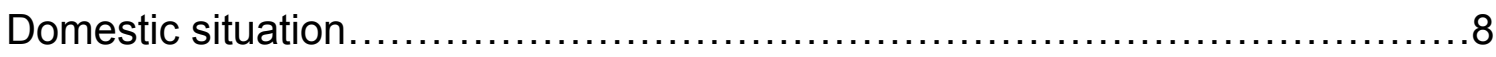

Comorbidities.....................................................................

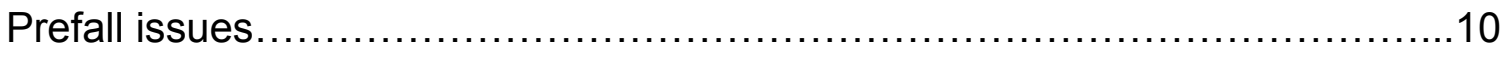

Dementia and confusion...........................................11

Diabetes mellitus....................................................

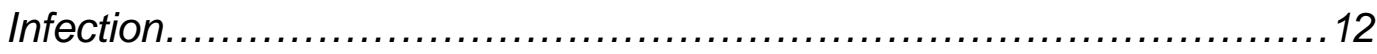

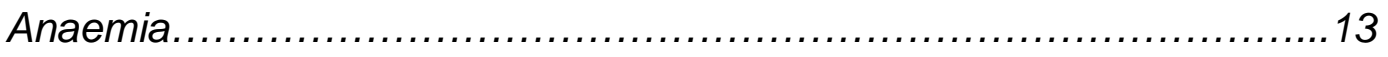

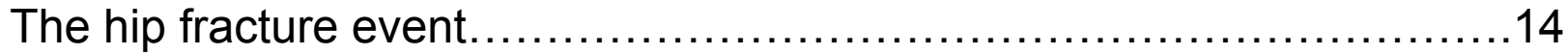

Pathophysiology of osteoporosis............................................. 14

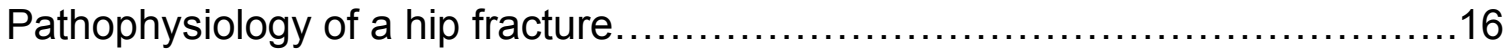




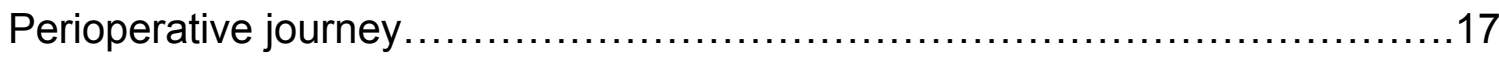

Orthopaedic assessment..............................................

Anaesthetic assessment............................................... 18

Orthogeriatric assessment.............................................. 19

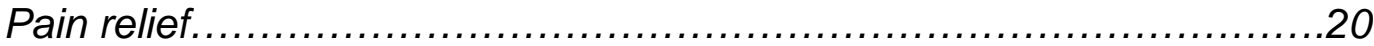

Surgical repair.........................................................

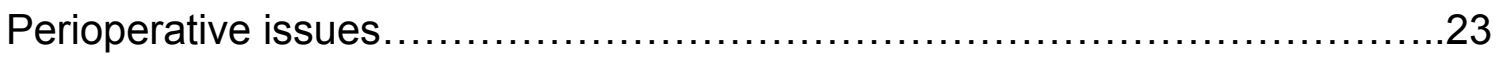

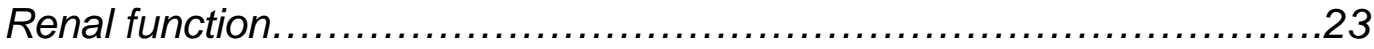

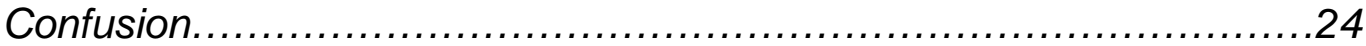

Malnutrition and fasting .................................................... 25

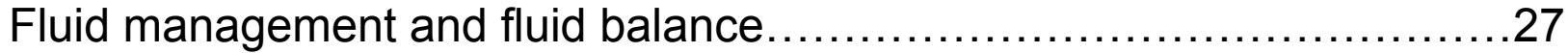

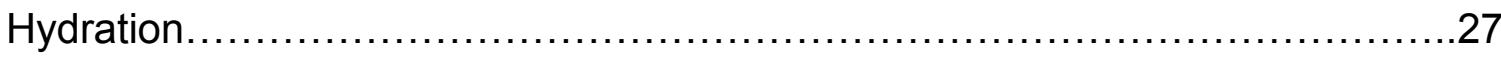

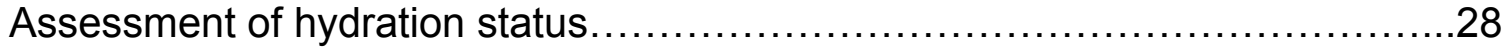

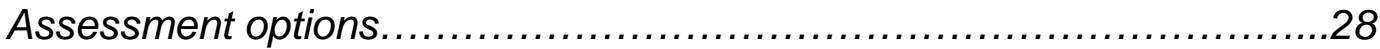

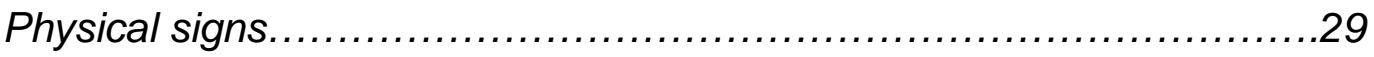

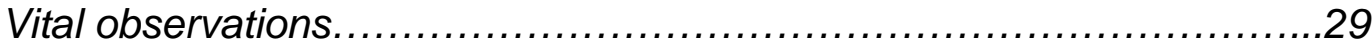

Body water............................................................

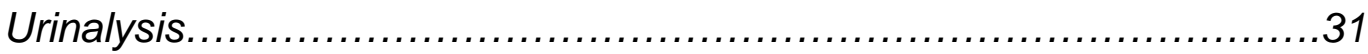

Biochemistry indicators............................................. 32

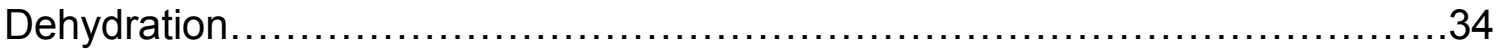

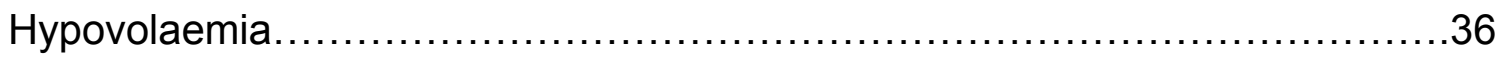

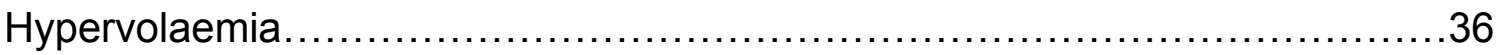

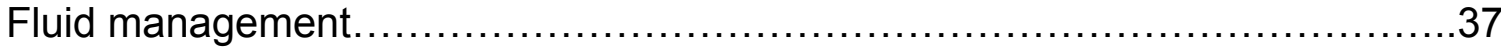

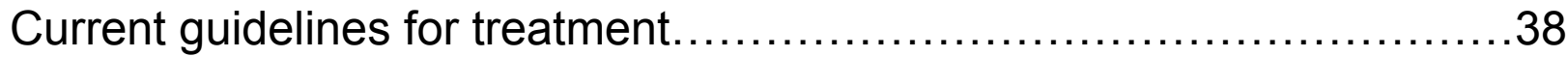

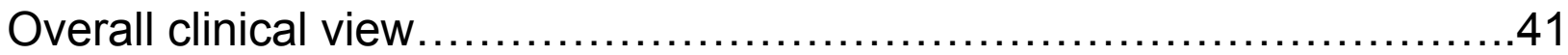

Purpose of further research............................................ 45 
Chapter 3: Methodology ......................................49

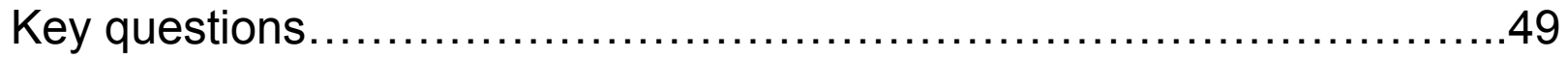

Definition of preoperative fluid management.............................49

Definition of postoperative outcomes..................................... 49

Ethical approval........................................................ 50

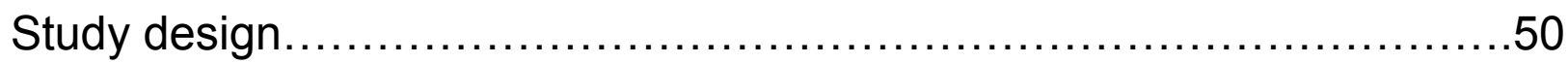

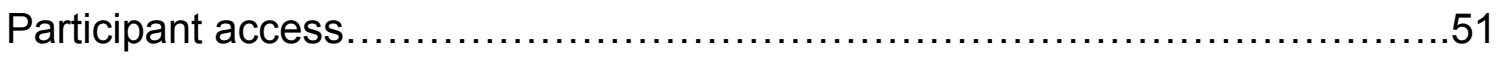

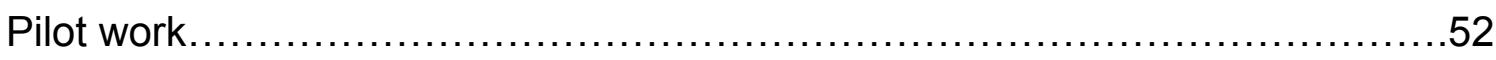

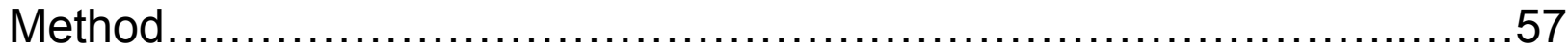

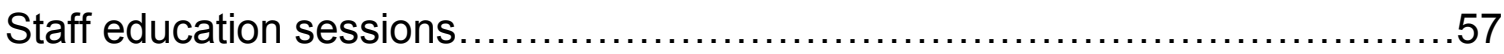

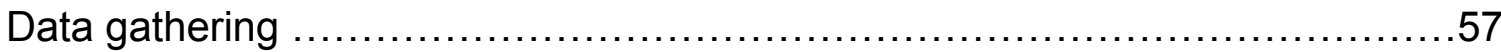

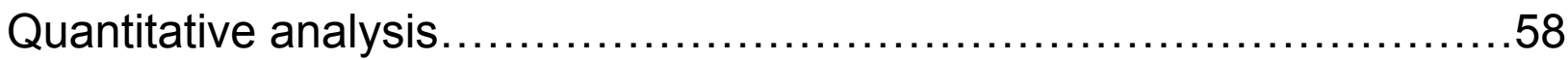

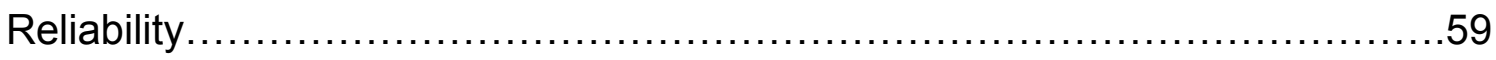

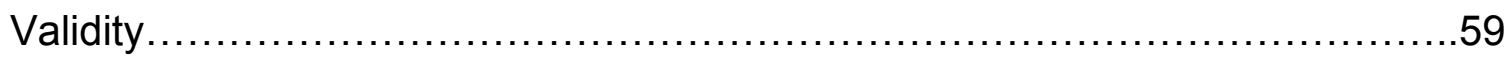

Chapter 4: Results.........................................60

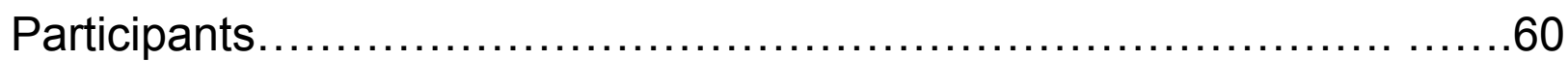

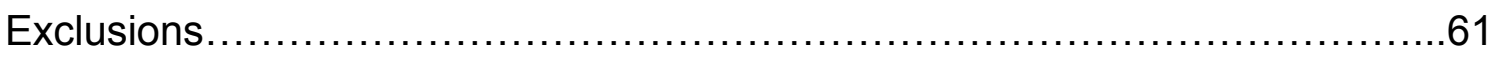

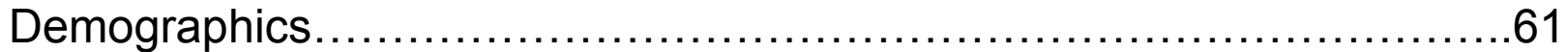

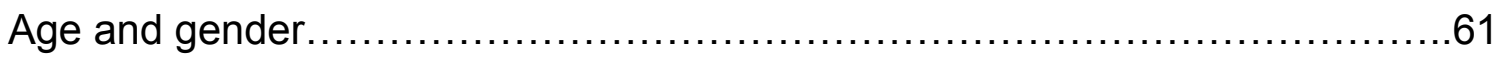

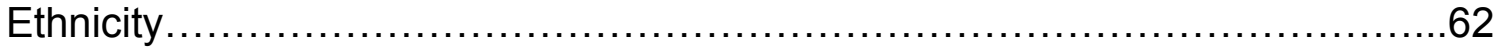

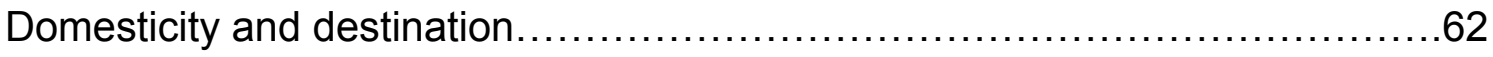

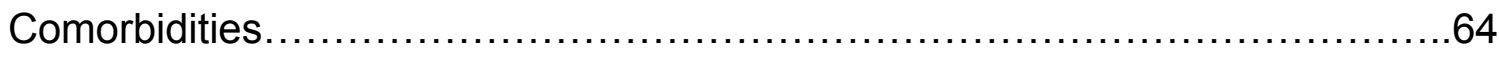

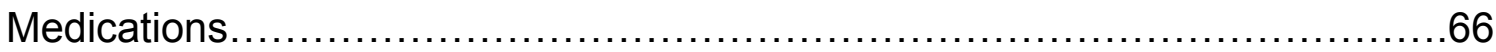

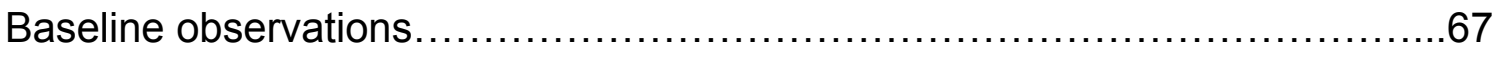

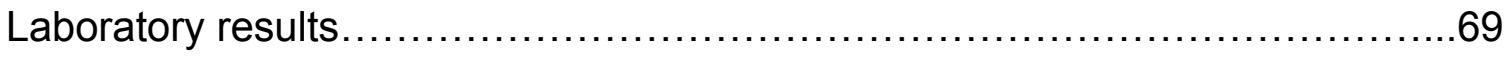

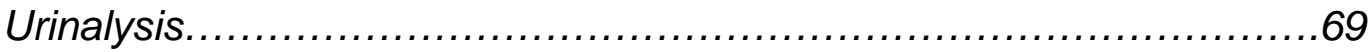

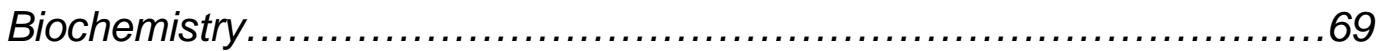

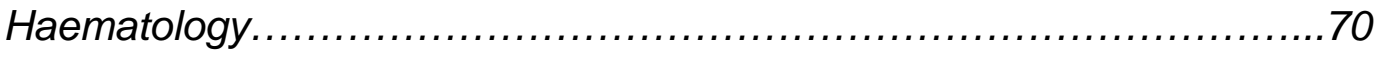




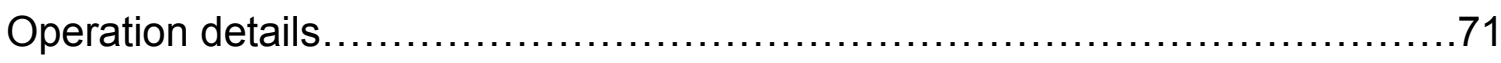

Delay to admission ................................................... 71

Delay to operation ...........................................................

Preoperative oxygen ................................................... 72

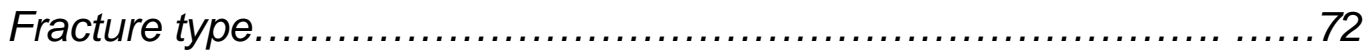

Preoperative traction.................................................. 72

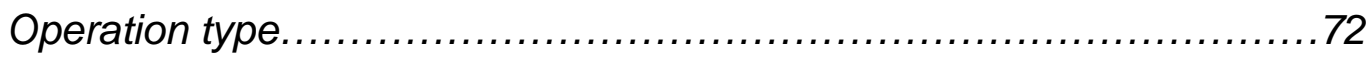

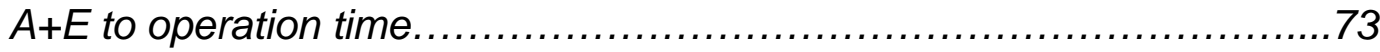

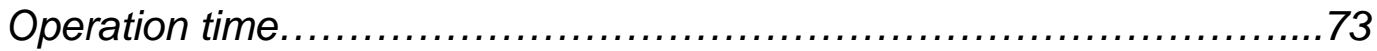

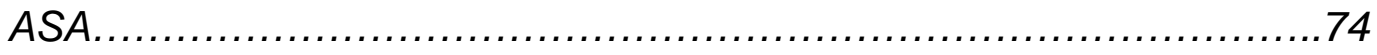

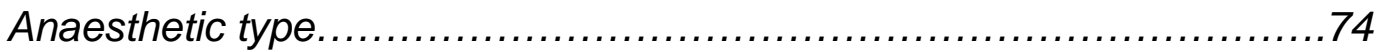

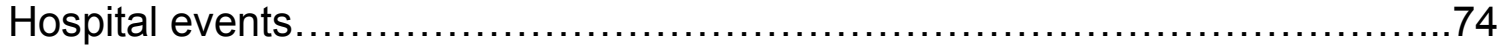

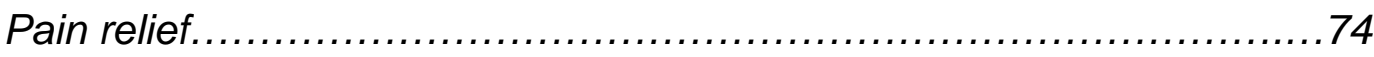

Orthogeriatric assessment........................................... 75

Indwelling catheter .................................................... 75

Dietitian referral...................................................... 76

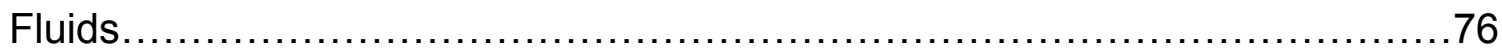

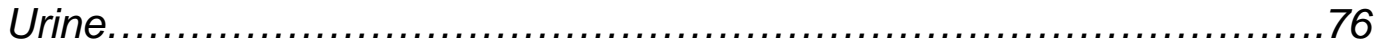

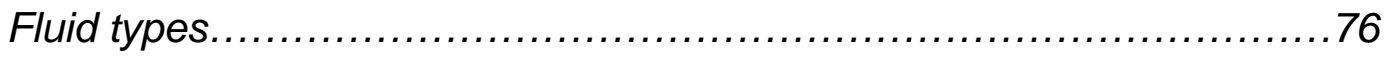

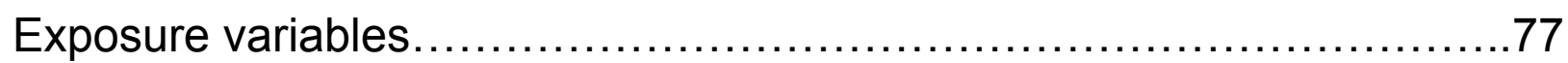

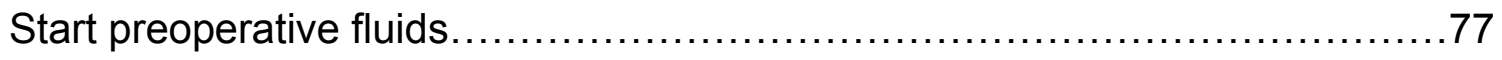

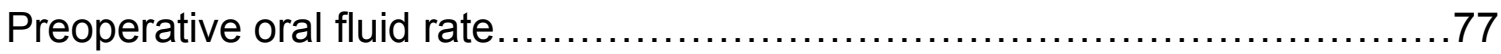

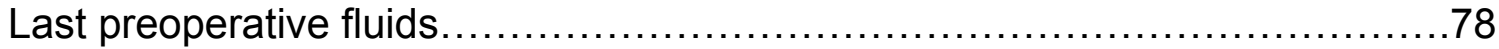

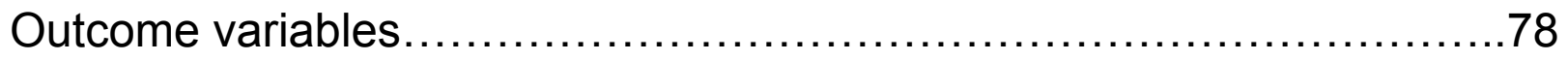

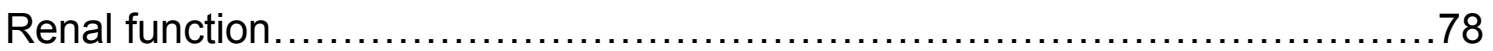

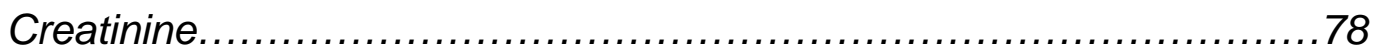

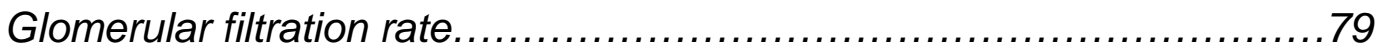

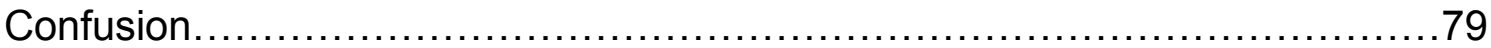

Postoperative intravenous discontinuation.................................... 79

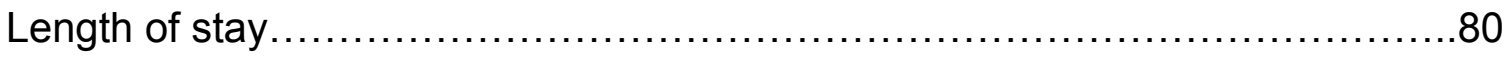




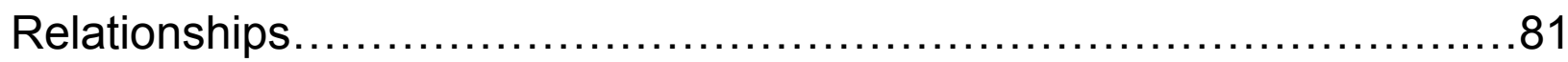

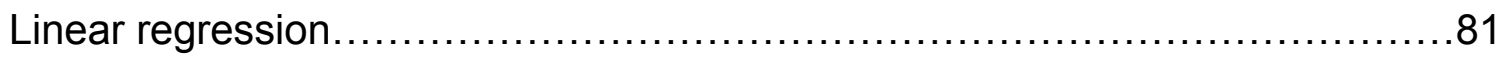

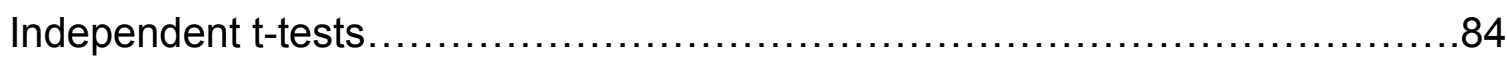

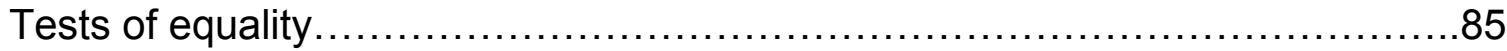

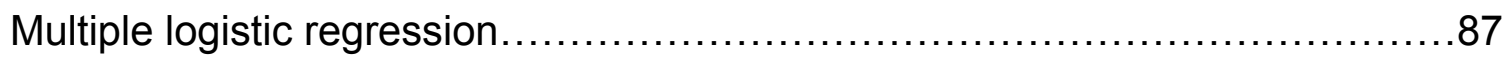

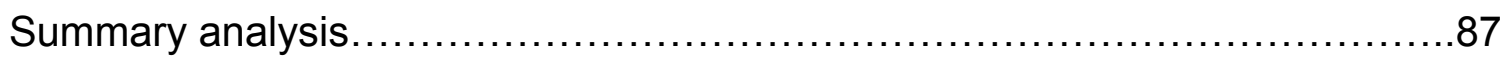

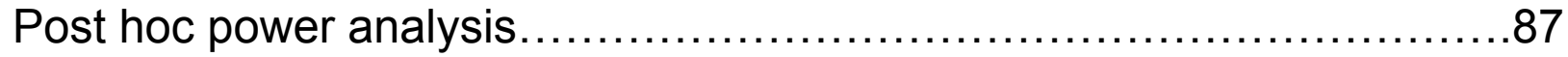

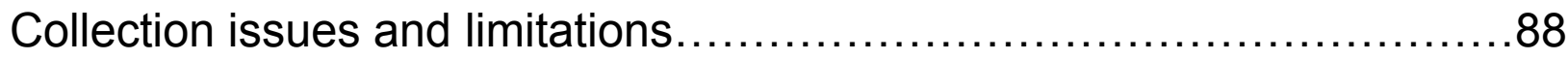

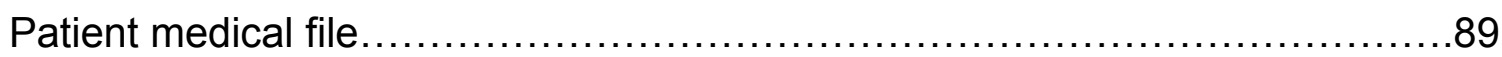

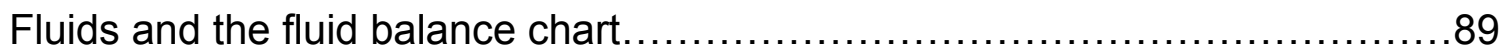

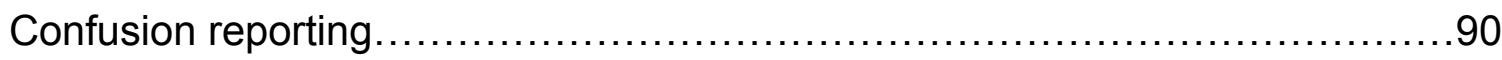

Chapter 5: Discussion....................................... 91

PFM and postoperative outcomes........................................ 91

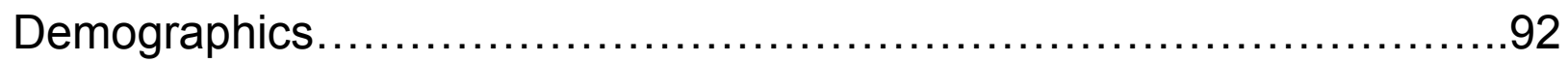

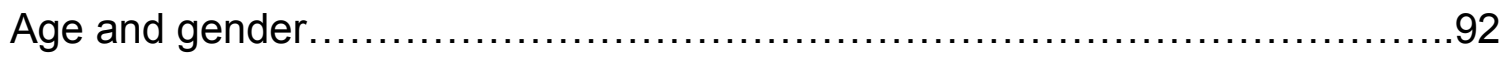

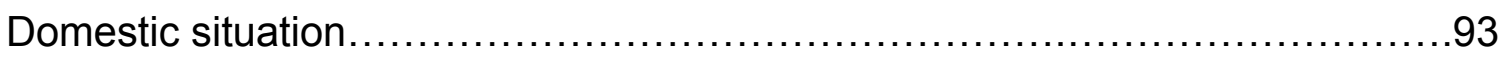

Comorbidities and prefall issues.............................................. 94

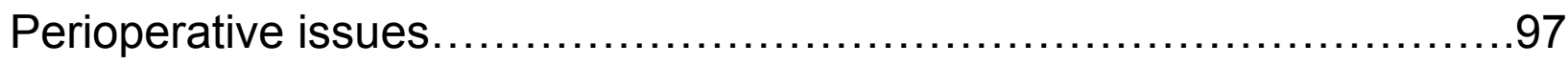

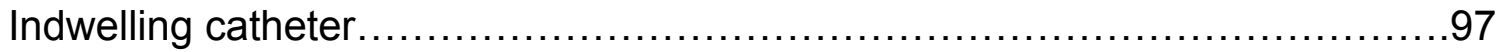

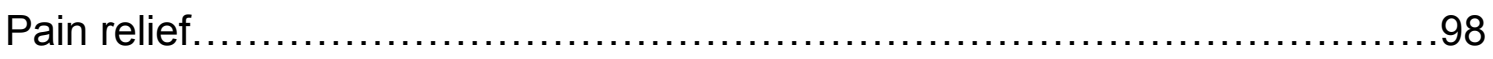

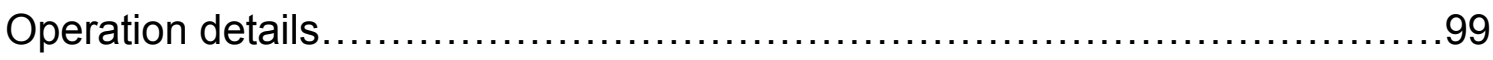

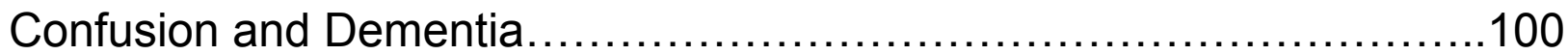

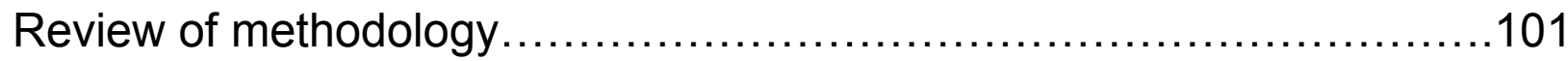

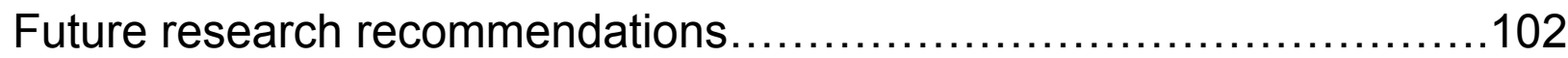

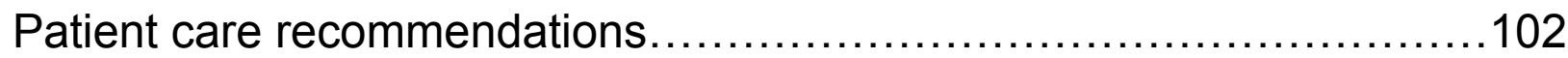

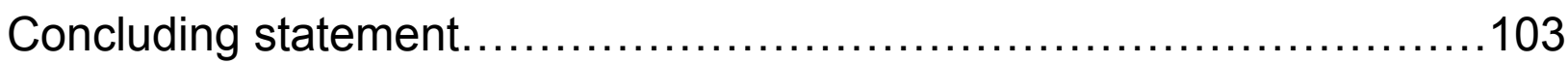




\section{Appendices}

Appendix A: Best practice evidence-based guideline summary: Acute management and immediate rehabilitation after hip fracture amongst people aged 65 years and over

(NZGG) 104

Appendix B: Perioperative fasting of adult patients (CCDHB) .......................108

Appendix C: Features of delirium and dementia (CCDHB) .........................112

Appendix D: Fractured neck of femur - Fast track form (CCDHB) ...................113

Appendix E: Adult vital signs chart sections (CCDHB) ............................114

Appendix F: Expedited review of observational studies ethical application.............116

Appendix G: Expedited review of observational studies ethical approval...............122

Appendix H: Research Advisory group (Maori) ethics approval.......................123

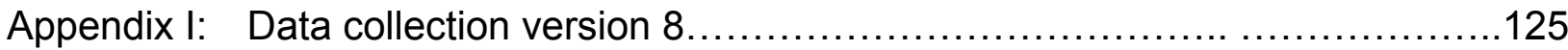

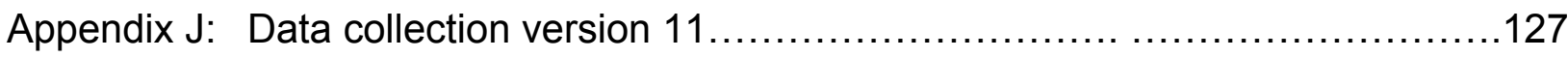

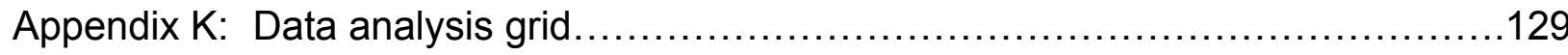

Appendix L: Poster for staff..........................................................

Appendix M: Sample (anonymous) patient data collection sheet.....................138

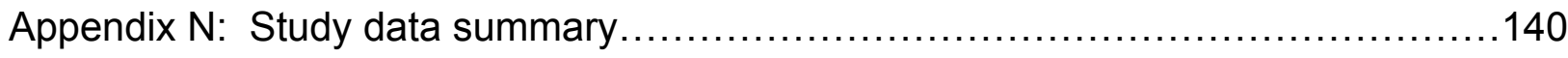

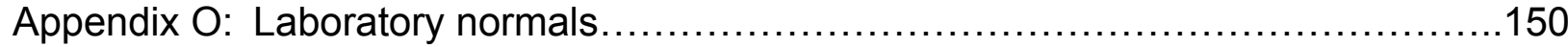

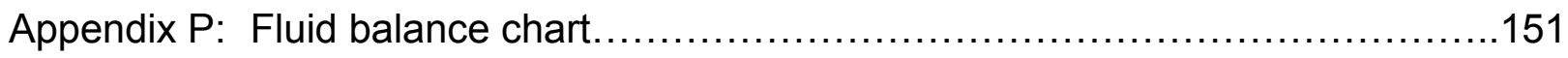

Appendix Q: General medicine - Fluid balance chart................................152

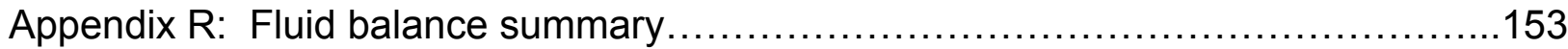

Appendix S: Confusion indicators.................................................... 154

References.................................................... 


\section{Tables}

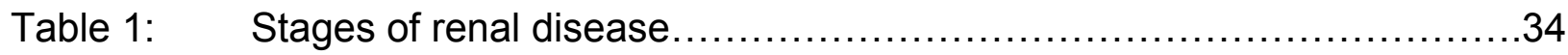

Table 2: $\quad$ NZ hip fracture patient statistics ........................................ 42

Table 3: $\quad$ ORSOS codes for hip fracture surgery by type........................52

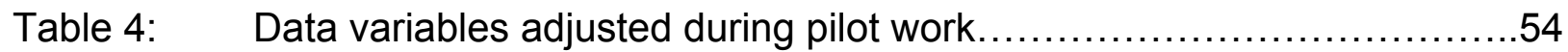

Table 5: American Society of Anaestheologists rating scale $\quad$.......................56

Table 6: Mean admission and 48hr postoperative baseline observations...........67

Table 7: $\quad$ Mean admission and $48 \mathrm{hr}$ postoperative data for biochemistry...........69

Table 8: $\quad$ Admission, $24 \mathrm{hr}$ and $48 \mathrm{hr}$ postoperative data for $\mathrm{Hb}$ and WCC..........70

Table 9: $\quad$ Mean preoperative, $24 \mathrm{hr}$ and $48 \mathrm{hr}$ postoperative hourly urine rate.......76

Table 10: $\quad$ PFM $v$ new postoperative confusion ..................................... 84

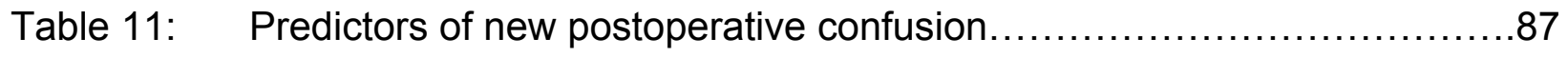




\section{Figures}

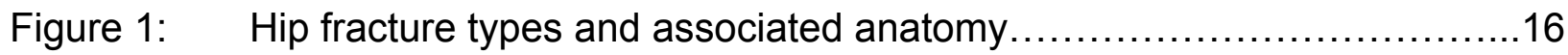

Figure 2: Hip fracture fixation devices.............................................

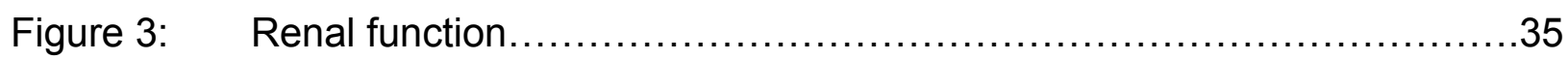

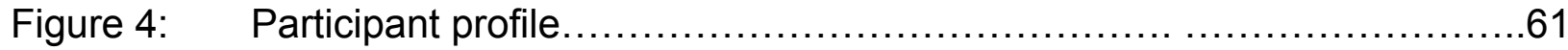

Figure 5: $\quad$ Males and females participants compared …..........................62

Figure 6: Domestic situation prior to admission....................................63

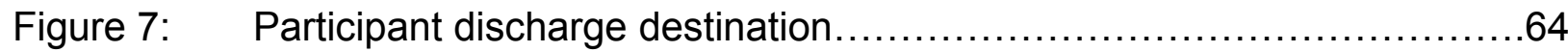

Figure 8: $\quad$ Patients comorbidity scores..............................................

Figure 9: $\quad A+E$ to operation time as per $6 \mathrm{hr}$ intervals............................73

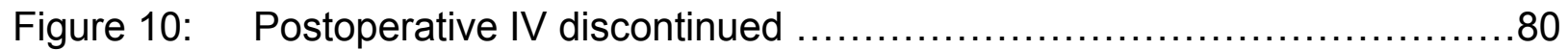




\section{Abbreviations}

\begin{tabular}{|l|l|}
\hline ADL & Activities of daily living \\
\hline AdDR & Admission to discharge record \\
\hline A+E & Accident and Emergency department \\
\hline ANOVA & Analysis of variables \\
\hline AnR & Anaesthetic record \\
\hline AVSC & Adult Vital Signs Chart \\
\hline ASA & American Society of Anaestheologists \\
\hline BMI & Body mass index \\
\hline BPsys & Systolic blood pressure \\
\hline CCDHB & Capital and Coast District Health Board \\
\hline CHF & Congestive heart failure \\
\hline CHS & Compression Hip Screw \\
\hline CORD & Chronic obstructive respiratory disease \\
\hline Cr & Creatinine \\
\hline CRD & Chronic renal disease \\
\hline CVD & Cerebrovascular disease \\
\hline CVP & Central venous pressure \\
\hline Deg C & Degrees Celsius \\
\hline DHS & Dynamic Hip Screw \\
\hline DM & Diabetes mellitus \\
\hline ESOL & English is a second oral language \\
\hline FBC & Fluid balance chart \\
\hline GA & General anesthetic \\
\hline GFR & Glomerula filtration rate \\
\hline Hb & Haemoglobin \\
\hline IDC & Indwelling urinary catheter \\
\hline IHD & Ischaemic heart disease \\
\hline JVP & Jugular venous pressure \\
\hline K & Potassium \\
\hline KRU & Kenepuru rehabilitation unit \\
\hline LOC & Level of consciousness \\
\hline LOS & Length of stay \\
\hline MedR & Medication record \\
\hline MDT & Multidisciplinary team \\
\hline mm HG & Millimeters of mercury \\
\hline Na & Sodium \\
\hline NBM & Nil by mouth \\
\hline NHI & National Health Identifier \\
\hline NOF & Neck of femur \\
\hline PADP & Patient admission to discharge plan \\
\hline
\end{tabular}




\begin{tabular}{|c|c|}
\hline PAR & Patient at risk \\
\hline PFM & Preoperative fluid management \\
\hline PNC & Peripheral nerve catheter \\
\hline $\mathrm{P}$ & Pulse \\
\hline PVD & Peripheral vascular disease \\
\hline RBC & Red blood cell \\
\hline $\mathrm{R}$ & Respirations \\
\hline $\mathrm{SpO}_{2}$ & Oxygen saturation \\
\hline$T$ & Temperature \\
\hline TBW & Total body water \\
\hline TIA & Trans-ischaemic attack \\
\hline TROCR & Trial of urinary catheter removal \\
\hline USG & Urine specific gravity \\
\hline UTI & Urinary tract infection \\
\hline WCC & White cell count \\
\hline WRH & Wellington Regional Hospital \\
\hline
\end{tabular}




\section{Glossary of terms}

\begin{tabular}{|l|l|}
\hline Albumin & $\begin{array}{l}\text { Main protein in blood that helps regulate } \\
\text { blood pressure }\end{array}$ \\
\hline Anaemia & $\begin{array}{l}\text { Lower than normal level of red blood cells } \\
\text { (RBC) }\end{array}$ \\
\hline Anaesthetic & $\begin{array}{l}\text { State of not feeling pain administered as a } \\
\text { general (whole body), spinal (around the } \\
\text { spinal cord), or epidural (into the epidural } \\
\text { space). }\end{array}$ \\
\hline Pnalgesic & Dein reducing medication \\
\hline Anorexia & Abnormal heart rhythm \\
\hline Arrythmia & Fatty lipid deposits on lining of arteries \\
\hline Atherosclerosis & Refilling speed in peripheral veins \\
\hline Bioimpedence & Measure of height versus weight ratio \\
\hline Body mass index (BMI) & Slower than normal heart beat \\
\hline Bradycardia & Generalised muscle wasting \\
\hline Cachexia & $\begin{array}{l}\text { Inflammatory disease of the heart and blood } \\
\text { vessels }\end{array}$ \\
\hline Cardiovascular disease & $\begin{array}{l}\text { Inflammatory bowel disease characterised } \\
\text { by ulcerations in the wall of the intestine }\end{array}$ \\
\hline Crohn's disease & $\begin{array}{l}\text { Ant disorder of the lungs that persistently } \\
\text { obstructs bronchial flow }\end{array}$ \\
\hline $\begin{array}{l}\text { Chronic obstructive respiratory } \\
\text { disease (CORD) }\end{array}$ & $\begin{array}{l}\text { Persistent disruption to normal function of } \\
\text { the kidneys }\end{array}$ \\
\hline Chronic renal disease (CRD) & Ability to think \\
\hline Cognitive function & $\begin{array}{l}\text { Diseases that together reduce individual } \\
\text { health }\end{array}$ \\
\hline Comorbidities & $\begin{array}{l}\text { Screw implant utilised for fixation of femoral } \\
\text { head fracture }\end{array}$ \\
\hline Compression Hip Screw (CHS & Acute cognitive dysfunction \\
\hline Confusion & $\begin{array}{l}\text { Inability of the heart to efficiently provide } \\
\text { blood to major organs }\end{array}$ \\
\hline Congestive heart failure (CHFion with or without hallucination \\
\hline Delirium & Water reducing \\
\hline Diuretic & (same as CHS) \\
\hline Dynamic Hip Screw (DHS) & Difficulty swallowing \\
\hline Dysphagia & Reduced respiratory rate \\
\hline Syspnoea & $\begin{array}{l}\text { Hormone produced in the kidney that } \\
\text { promotes the production of RBC in the bone } \\
\text { marrow }\end{array}$ \\
\hline Electrolytes & \\
\hline Erythropoietin & \\
\hline
\end{tabular}




\begin{tabular}{|l|l|}
\hline Femur & Long bone of the thigh \\
\hline Hypo / hyperalbuminaemia & Low / high level of plasma albumin \\
\hline Glomerula filtration rate (GFR) & $\begin{array}{l}\text { Rate at which the glomerular of the kidneys } \\
\text { filters blood and other fluids }\end{array}$ \\
\hline Head of femur & $\begin{array}{l}\text { Spherical bone structure at the distal end of } \\
\text { the femur }\end{array}$ \\
\hline Haematological & Associated with the blood \\
\hline Hemiarthroplasty & $\begin{array}{l}\text { Partial replacement of the hip joint involving } \\
\text { the trochanter and head of the femur }\end{array}$ \\
\hline Hip fracture & $\begin{array}{l}\text { Fracture of the femur associated with the } \\
\text { head or neck or upper shaft }\end{array}$ \\
\hline Hydration & State of being hydrated or water balance \\
\hline Hyper/hypotension & Higher/lower than normal blood pressure \\
\hline Hypervolaemia & Higher than normal circulating blood volume \\
\hline Hypotension & Lower than normal blood pressure \\
\hline Hypovolaemia & Lower than normal circulating blood volume \\
\hline Hypoxia & Low level of oxygen \\
\hline Interstitial & Within tissue \\
\hline Level of consciousness (LOC) & State of reaction to sight, sound and pain \\
\hline Metabolic acidosis & Raised plasma pH (more acidic) \\
\hline Metabolism & $\begin{array}{l}\text { Energetic reactions within cells during } \\
\text { normal living }\end{array}$ \\
\hline Morbidity & Rate of acquired illness or disease \\
\hline Mortality & Death rate \\
\hline Multidisciplinary team & $\begin{array}{l}\text { Team comprising of medical, nursing and all } \\
\text { allied health professionals }\end{array}$ \\
\hline Neck of femur & $\begin{array}{l}\text { Bone structure between the shaft of femur } \\
\text { and the head of the femur }\end{array}$ \\
\hline Death of living cells \\
\hline Necrosis & Connection between nerves and muscles \\
\hline Neuromuscular & Swelling of the tissues \\
\hline Oedema & $\begin{array}{l}\text { Pertaining to the boney structures of the } \\
\text { body called the skeleton }\end{array}$ \\
\hline Orthopaedic & Bone forming cells \\
\hline Osteoblasts & Bone breakdown cells \\
\hline Osteoclasts & End of life care \\
\hline Palliative & Under the skin \\
\hline Percutaneous & $\begin{array}{l}\text { Combined pre- intra-, and postoperative } \\
\text { period }\end{array}$ \\
\hline Perioperative & $\begin{array}{l}\text { Blood circulation to the limbs, hands and } \\
\text { feet }\end{array}$ \\
\hline Plasma & $\begin{array}{l}\text { Atherosclerosis of the arteries of the } \\
\text { extremities }\end{array}$ \\
Blood related \\
\hline Peripheral perfusion & \\
\hline Peripheral vascular disease & \\
\hline
\end{tabular}




\begin{tabular}{|l|l|}
\hline Polypharmia & Multiple medication usage \\
\hline Polyuria & Excess urine production \\
\hline Proximal & Nearest to upper body \\
\hline Pulmonary & Pertaining the lungs \\
\hline Red blood cells (RBC) & Element of the blood that carries oxygen \\
\hline Renal & $\begin{array}{l}\text { Relating to the kidneys. Renal failure, or } \\
\text { evidence of, prevents to effective filtration } \\
\text { process associated with kidneys }\end{array}$ \\
\hline Skin turgor & $\begin{array}{l}\text { Elasticity of the skin recognised by pinching } \\
\text { small areas of the skin together }\end{array}$ \\
\hline Solute & Dissolvable substances \\
\hline Stroke & $\begin{array}{l}\text { Blockage of blood flow within the brain } \\
\text { causing death of brain tissue }\end{array}$ \\
\hline Tachycardia & Faster than normal pulse \\
\hline Total body water (TBW) & Used in the calculation of hydration status \\
\hline Ulcerative colitis & $\begin{array}{l}\text { Inflammatory bowel disease with ulcers of } \\
\text { the lining of the colon }\end{array}$ \\
\hline Urea & $\begin{array}{l}\text { Product of the breakdown (metabolism) of } \\
\text { protein }\end{array}$ \\
\hline Urinalysis & $\begin{array}{l}\text { Analysis of elements of excretion and } \\
\text { volume of urine }\end{array}$ \\
\hline Urine Specific Gravity (USG) & $\begin{array}{l}\text { Urine test that measures the concentration } \\
\text { of all chemical particles }\end{array}$ \\
\hline Relating to arteries and veins \\
\hline Vascular & Dilation of blood vessels \\
\hline Vasodilation &
\end{tabular}




\section{Chapter 1: Introduction}

The key question that will be posed in this thesis is - Does preoperative fluid management of the older adult patient admitted with a hip fracture have a relationship with outcomes of:

a) Postoperative renal function, defined as significant change in GFR and creatinine from admission to 48 hour postoperative.

b) Diagnosis of new reported confusion within 48 hour postoperatively.

c) Timing of discontinuation of postoperative intravenous (IV) fluids, indicative of resumption of normal diet.

d) Total length of stay (LOS) in hospital.

This introduction (chapter 1) provides an overview of the thesis. Chapter 2 discusses current research around the topic as it relates to the planned outcomes to be measured. Chapter 3 will discuss the quantitative analysis and biostatistical process; the study design; data collection methods and management; and explanation of the data analysis plan. Chapter 4 will provide descriptive statistics of the gathered data, tables and figures to provide pictorial illustrations and comments on the relationships between variable data. Chapter 5 will discuss the findings as they relate to the designated perioperative outcomes of the study, and whether this is comparable to current research outlined in chapter 2; provide recommendations for effective fluid management; and suggestions regarding further research.

\section{Background}

Falls in the older adult aged 65 years and over are common in New Zealand (NZ) with $43 \%$ of reported incidents annually requiring admission to hospital in this age group (Accident Compensation Corporation, 2005). These statistics can be further described by saying that $55 \%$ of these patients who are aged $65-69$ years, $65 \%$ aged $70-74$ years, and $82 \%$ aged older than 75 years who have had 
a fall are admitted to hospital. Many of these patients suffer a hip fracture with approximately 200 of this type of patient admitted each year to Capital and Coast District Health Board (CCDHB) (Capital and Coast District Health Board Decision Support Unit, 2012), and over 3000 admitted nationally in NZ (Ministry of Health, 2002). The approximate age for this cohort of hip fracture patients is 83 years (Ministry of Health, 2002), with the twelve month mortality rate in NZ for hip fracture patients has been reported as being between 19 and 32\% (Davison, Merrilees, McKie, Sainsbury, \& Gilchrist, 2001; Thwaites, Mann, Gilchrist, McKie, \& Sainsbury, 2007; Young, Seigne, Bright, \& Gardner, 2006). This is greatly above the national average for this age group of approximately $7-10 \%$ (Statistics New Zealand, 2007).

Hip fracture incidents are usually caused by an accidental fall, or collapse, and are assisted by bones of the older adult being osteoporotic (have reduced calcium and density) and more easily fractured. The patient may have fallen due to being unsteady on their feet because of a range of medical conditions, or comorbidities, such as Parkinson's disease or stroke. The incident may also be due to a range of other medical issues, such as a urinary tract infection (UTI), pneumonia, or polypharmia (multiple medication usage) (Rai \& Mulley, 2007).

The hip fracture patient may be alone when the incident occurs and not be able to call for help or assist themselves in any way. Emergency services may not, therefore, attend immediately, and the patient may be lying on the floor without refreshment for quite some time. They may also be in a weakened state due to an existing illness or haemorrhage from the fracture site (Nichol, Wilson, \& Webster, 2008). Patients may, therefore, become dehydrated which adversely affects their cognitive and renal function (Marieb \& Hoehn, 2007). Patients presenting with a hip fracture may be in pain; $30-60 \%$ may be dehydrated and malnourished (Hanger, Smart, Merrilees, \& Frampton, 1999; Mentes, 2006; Nematy, Hickson, Brynes, Ruxton, \& Frost, 2006); suffer from possible hearing and vision issues; and be in an unfamiliar environment on admission to hospital. 
The journey for most hip fracture patients through the hospital system will almost always involve a surgical repair of the hip fracture, but a very few palliative care patients will be treated conservatively by placement in a hip cast or restraint. This journey will involve waiting in the emergency department; tests to determine the state of the injury and diagnosis of hip fracture or other condition; and the requirement to be fasted, or nil by mouth (NBM). Nil by mouth is a traditional requirement for patients waiting for surgery, but in recent years there has been debate about the length of time required, e.g. 2-6 hours for fluids, and 8-12 hours for solid food (Power, et al., 2012).

The total average length of stay (LOS) of the older adult hip fracture patient at CCDHB Wellington Regional Hospital (WRH) was 9.9 days for the fiscal year 2009, 8.03 days for the year 2010, and 7.13 days for the year 2011 (Capital and Coast District Health Board Decision Support Unit, 2012). Within other District Health Board (DHB) tertiary hospitals in NZ there is an approximate LOS ranging between 7.3 and 10 days (Ministry of Health, 2002). This is comparable with other western countries such as Sweden where the LOS in a comparable facility was 9 days (Gunnarsson, Lonn, \& Gunningberg, 2009), and 10 days in Hong Kong (Leung, et al., 2011). Quoted figures will be dependent on variations in discharge to rehabilitation environment practices between hospitals. The immediate postoperative period for the surgical hip fracture patient involves ensuring mobility and self-cares assessment to gauge rehabilitation requirements. This may include immediate discharge to own home, specified rehabilitation setting, another residential setting, or to other hospital level care.

Current treatment and care protocol of older adult hip fracture patients admitted to CCDHB follow the guidelines - Acute management and immediate rehabilitation after hip fracture amongst people aged 65 years and over produced by the New Zealand Guidelines Group (NZGG) (2003) (Appendix A). These guidelines state that there should be careful fluid management to reduce 
risk of dehydration, overload, or electrolyte imbalance (New Zealand Guidelines Group, 2003). Exact definitions for this statement are, however, not provided. Surgical patients at CCDHB are also cared for within the parameters of the Perioperative fasting of adult patients policy (Capital and Coast District Health Board, 2011) (Appendix B). This study will assess how well CCDHB complies with these policies and assess the consequence of deviation. The results will inform a more useful working definition of the terms of the guidelines and policy definitions.

Effective management of fluid balance, or hydration, in the older adult surgical patient is paramount in minimising adverse outcomes (Ward, 2011). Effective fluid management, which includes oral and intravenous (IV) fluids, reduces the risk of dehydration, and ensures optimal patient health prior to undergoing anaesthesia for surgical repair of the hip fracture. Key elements that may affect good outcomes in this cohort, and be affected by dehydration, are renal and cognitive function. Renal function declines when the patient is dehydrated, and cognitive function is known to be adversely affected by dehydration (Bennett, Thomas, \& Riegel, 2004; Marieb \& Hoehn, 2007).

Confounding issues associated with this cohort of patients include malnourishment and dehydration prior to the hip fracture event (Hanger, et al., 1999; Nematy, et al., 2006). Many older adults live alone or are totally housebound (Bennett, et al., 2004; Nichol, et al., 2008) and the risk of mortality is higher when nutritional status is substandard (Kagansky, et al., 2005; Mentes, 2006).

\section{Purpose of thesis}

The ultimate aim of this study is to provide new information about the relationship between preoperative fluid management and postoperative renal function, reported new confusion, timing of discontinuation of postoperative IV fluids, and 
LOS, in order to better define careful fluid management of the older adult hip fracture patient.

Many older adult hip fracture patients are dehydrated and malnourished when they are admitted. They are required to be fasted or have minimal preoperative fluids and patients often wait for more than two days for surgery (Bottle \& Aylin, 2006). Many researchers have investigated ways of improving outcomes for hip fracture patients, and guidelines have consequently been produced (National Clinical Guideline Centre, 2011; New Zealand Guidelines Group, 2003).

While careful fluid management is recommended by the NZGG (2003) no specific recommendations regarding management of and relationships between fluid management and patient outcomes have been studied. Hence data supporting current fluid management practice are urgently required.

This study researched practice at CCDHB according to current inpatient, national (New Zealand Guidelines Group, 2003), and internationally (Handoll, Queally, \& Parker, 2011; National Clinical Guideline Centre, 2011) accepted guidelines, in order to ascertain deviation from and consequences of deviations from these guidelines. Current local information about the management of this cohort of patients will be provided as an outcome of this study.

Patient care information gathered in this study based involved patients aged 65 years and over who were admitted to CCDHB with a diagnosis of hip fracture and who underwent surgery over a period of approximately six month. Data collection included recording patient information from admission to 48 hours postoperatively, focusing on renal function, oral and IV fluids, reported new onset postoperative confusion and LOS. Cohort characteristics were considered as possible confounders, which included age, gender and comorbidity score. Information was extracted from clinical records and correlation and regression analysis techniques were used to consider relationships between preoperative 
fluid management, and designated outcomes, with some adjustment for possible confounding variables. The data set included 100 patients, which is approximately $50 \%$ of the total hip fracture patients admitted to CCDHB annually.

The reported research study will finish by offering recommendations for future best practice where possible. 


\section{Chapter 2: Background}

\section{The older adult patient}

The older adult is commonly defined as a person who is over 65 years of age. Statistical information shows that between 10 and $16 \%$ of the population (depending on regional area) in the 2006 New Zealand census fell into this age range, with $55 \%$ women and $45 \%$ men (Statistics New Zealand, 2007). This is an increase of 45,000 people since the 2001 NZ Census, and the size of this cohort of older adults is expected to peak within twenty years to double the current numbers. These figures are comparable with other western countries, where older adults make up $16 \%$ of the population in the United Kingdom (UK) (Rai \& Mulley, 2007) and 12.9\% in the United States (Department of Health and Human Services, 2011). The population in NZ of those aged 85 years and older is approximately $12 \%$.

\section{The older adult hip fracture patient}

Studies describing the demographics of hip fracture patients indicate that the average age is approximately 83 years (Foss, Jensen, \& Kehlet, 2007; Ministry of Health, 2002; National Clinical Guideline Centre, 2011), and 72-87\% of this type of fracture is sustained by women (Fergus, Cutfield, \& Harris, 2011; Nematy, et al., 2006). There is a relatively high chance of people in the older adult age group sustaining a hip fracture as approximately $50 \%$ of women and $30 \%$ of men in NZ will sustain a fracture associated with osteoporosis in their lifetime (New Zealand Orthopaedic Association, 2003). In NZ there are over 3,000 hip fractures reported annually (Ministry of Health, 2002). A hip fracture is usually caused by an accidental fall, or collapse, and is due in part to the fact that the elderly bones are osteoporotic (have reduced calcium and density) and are more easily broken (Rai \& Mulley, 2007). 
The effect on the older adult patient who has sustained a hip fracture may be systemic, causing a range of pathophysiological changes, or even death (Connor, Langley, \& Cryer, 2006). The mortality rate for this group of patients ranges between $12 \%$ at 30 days (Young, et al., 2006) and approximately $20 \%$ at six month (Fergus, et al., 2011), but the mortality rate of $16-33 \%$ at $12 \mathrm{mth}$ (Davison, et al., 2001; Fergus, et al., 2011; Ministry of Health, 2002; Roche, Wenn, Sahota, \& Moran, 2005) is three to four times the expected rate of $7-10 \%$ for this age group (Statistics New Zealand, 2007).

Further issues that relate to the older adult hip fracture patient are that they are bedridden and out of their normal environment. This may cause disorientation or confusion; they may have reduced cognitive function or increasing dementia; or they may be in pain. They may also have other medical issues, for example diabetes mellitus (DM), where the fall may be hypoglycaemic related. In a study of 400 patients aged 65 years of age and over who were admitted to a geriatric ward for general assessment in a Singapore hospital it was shown that $28 \%$ exhibited symptoms of confusion, $49 \%$ had a urinary tract infection (UTI), $45 \%$ were in pain, and $23 \%$ had a chest infection (Faezah, Zhang, \& Yin, 2008).

\section{Domestic situation}

Approximately $80-90 \%$ of older adults live alone, often housebound (approximately 20\%), and social isolation is known to be a factor in poor eating and drinking habits (Nichol, et al., 2008). The proportion of older adult hip fracture patients admitted from their own home is between $61-88 \%$ (Fergus, et al., 2011; Nematy, et al., 2006), compared with residential care of between 2139\% (Fergus, et al., 2011; Foss, et al., 2007). In a convenience study of 25 patients admitted to hospital with hip fracture Nematy, et al. (2006) found that $56 \%$ were malnourished on admission. Of these patients $88 \%$ had come from their own home, and $64 \%$ had been living alone. In two studies of older adult (referred to as elderly) patients admitted with hip fracture it was also found that 
average serum albumin levels were $36 \mathrm{~g} / \mathrm{L}$ (acceptable norm is $38-48 \mathrm{~g} / \mathrm{L}$ ) which is indicative of malnutrition risk (Foss, et al., 2007; Nematy, et al., 2006).

Dehydration is an associated issue with a large number of older adult patients being dehydrated on admission. They may have come from a residential care environment, where Mentes (2006) found in a study of 35 nursing home residents that $31 \%$ were dehydrated at least once during a six month period, or community environment where Bennett, et al. (2004) found that from 148 patients admitted to an emergency department $44 \%$ were dehydrated.

Environmental and personal factors play a large part in the incidence of hip fracture, and admissions to hospital due to a fall are common in the older adult (Accident Compensation Corporation, 2005) who may be unstable or have limited mobility for a number of reasons. The cohort may trip easily due to inadequate eye sight; have decreased cognitive function that adversely affects their ability to navigate their journey; and have gait (walking ability) and balance issues. The cohort is further characterised by being unstable due to a generalised weakness or inherent disease, such as Parkinson's disease, or previous disease process, such as a stroke, causing a generalised one sided weakness (Rai \& Mulley, 2007). Further risk factors that predispose the older adult to a fall may include reduced energy due to an inability to breathe adequate volumes of air because of chronic obstructive respiratory disease (CORD). They may also have balance issues due to the effects of an ear infection, have recurrent knee pain from an old injury or osteoarthritis in their joints, or the fall may simply be unexplained. Lack of regular exercise will also cause muscle wastage, and general fragility will inhibit the ability to provide self-support if an incident did occur. Programs designed to enhance strength in the community based older adult have been recommended as most falls occur within the home or residential setting for this age group (Accident Compensation Corporation, 2005). 


\section{Comorbidities}

Hip fracture patients may have a number of co-existing comorbidities or medical issues. This cohort of patient may also have an initial diagnosis of hip fracture but on further investigation has other untreated systemic issues.

The older adult hip fracture patient may present with comorbidities such as congestive heart failure (CHF) or renal impairment (kidney dysfunction). They may also have pneumonia, or be hypertensive (high blood pressure), which may impact upon renal function (Rai \& Mulley, 2007). This could be because many older adults do not see a doctor regularly due to reduced mobility, finances, or simply because they see their symptoms as a result of ageing (Rai \& Mulley, 2007). Multiple and complex medical and social issues may therefore be apparent when the older adult hip fracture patient is admitted to hospital.

Fergus, et al. (2011) found that $69 \%$ of hip fracture patients have an American Society of Anaestheologists (ASA) rating of III, which is indicative of severe systemic disruption of more than one system of the body, for example, cardiac and pulmonary, or complications from chronic DM (Gwinnutt, 2008). Comorbidities common in this cohort of patients that are also significantly associated with fracture risk are Parkinson's disease, multiple sclerosis, chronic obstructive respiratory disease (CORD), osteoarthritis, and heart disease (Dennison, et al., 2012). All these conditions are associated with osteoporosis (Marieb \& Hoehn, 2007).

\section{Prefall issues}

Causative issues relating to falls in the older adult are varied and often inconclusive. Progressive cognitive impairment, existing confusion, or other acute medical issue such as DM, infection or anaemia may be present. 


\section{Dementia and confusion}

A common progressive and disabling cognitive disease of the older adult is dementia which predominantly occurs after 65 years of age (World Health Organisation, 2012). The signs of dementia are associated with diminished higher level thinking and cognitive decline has been noted in $28-43 \%$ of hip fracture patients (Foss, et al., 2007; Juliebø, et al., 2009). Signs of dementia also include loss of control of emotional, social and motivational behaviors, with the depth of symptoms varying between individuals (Rai \& Mulley, 2007).

Symptoms may include confusion and poor insight about their current situation; inappropriate behaviour or conversation; or being unkempt or apathetic (Capital and Coast District Health Board, 2007). Patients suffering from dementia need to be cared for with extreme thoughtfulness and quiet appraisal, so that their symptoms are not aggravated (World Health Organisation, 2012). Nursing management of these patients needs to focus on regular contact and routines, incorporating the patient in decision making, and creating a hazard free environment (Bowker, Price, \& Smith, 2006).

Older adult patients may become confused when hospitalised and will exhibit an acute onset in behaviours similar to dementia patients but there will be fluctuation in intensity. The difference with confused patients is that they may be delirious and have hallucinations; their sleep-wake cycle is often disrupted; attention span is often irregular; and they will have some lucid periods of complete orientation to their situation .(Bowker, et al., 2006; Capital and Coast District Health Board, 2007).

Confusion associated with dementia, which is difficult to assess for the untrained healthcare worker (Fick, Hodo, Lawrence, \& Inouye, 2007), may develop into a difficult patient management situation. Patients will be acutely confused, when previously orientated to their situation, and possibly be less alert or sleepier at inappropriate times. Symptoms of confusion and dementia are outlined in a table within the CCDHB policy Dementia Guidelines (Capital and Coast District Health 
Board, 2007) (Appendix C) entitled Differential diagnosis of dementia. This cohort of patients has poorer outcomes after a hip fracture with generally longer stays in hospital and early mortality (Juliebø, et al., 2010). Added to this scenario is the fact that hip fracture patients who have suffered an episode of confusion or delirium and do not have a diagnosis of dementia may have a higher chance of developing dementia or dying in the subsequent five year period. Hip fracture patients who also have DM are also more likely to have a delirium or episode of confusion (Lundstrom, Edlund, Bucht, Karlsson, \& Gustafson, 2003) due to disruption of their food and insulin regime causing a hypoglycaemic event.

\section{Diabetes mellitus}

A common medical issue in older adults of DM is a condition that affects the body's ability to metabolise glucose. Insulin, normally produced in the pancreas, is often administered artificially to the patient to assist in this process. The condition often goes undetected if the onset is slow, and associated symptoms may be weight loss, or incontinence (Bowker, et al., 2006). Systemic indications of DM may also be peripheral neuropathy or pneumonia, and the patient may be prone to infections because of poor circulation from peripheral vascular disease (PVD) (Rai \& Mulley, 2007). Diabetic patients require around the clock management of their condition, ensuring that insulin levels and food intake is appropriately managed. Both patients with DM Type 1 that require insulin replacement, and those with DM Type 2, with or without insulin dependency, may be well equipped to manage the healing process post hip fracture, but focusing on adequate dietary supplements is a necessity (Bowker, et al., 2006).

\section{Infection}

An undetected infection may have been the essential precursor to the fall, which culminated in the admission to hospital with a hip fracture. It is often overlooked in the initial assessment of the patient unless it is obvious, such as in pneumonia or infected cellulitis. A fever may be absent, but the patient's family may say the patient is not functioning normally, and that the patient is slightly dehydrated 
(Bowker, et al., 2006). Rai and Mulley (2007) write about the high incidence of untreated urinary incontinence in the UK associated with the older adult living in their own home, and subsequent high rates of UTI, but that diagnosis in hospital is often flawed by contaminated specimens after admission (Bowker, et al., 2006). Infection may, therefore, be an undetected contributor to the hip fracture event and also may impact on renal function postoperatively.

After review of blood results an elevated serum white cell count (WCC) is usually an indication of infection, but analysis of the different types of WCC (for example, neutrophils, lymphocytes, or eosinophils) may in fact indicate any inflammation, a range of cancers, an unusual drug response, allergy, or bone marrow failure (Longmore, Wilkinson, Turmezei, \& Cheung, 2007). A relatively normal WCC may also mask a current infection (Bowker, et al., 2006). A progressing undetected infection will, however, affect the patient, precipitating confusion, dehydration, poor healing, systemic blood poisoning, and reduced ability to cope with rehabilitation postoperatively (Rai \& Mulley, 2007). The older adult's immune system is also less functional, which will slow the recovery process from any infection.

\section{Anaemia}

The older adult hip fracture patient may have reduced plasma haemoglobin $(\mathrm{Hb})$ due to dietary issues (such as reduced red meat intake causing iron deficiency anaemia); less production of the hormone erythropoietin in the kidneys which stimulates bone narrow to produce red blood cells (RBC); or a gastric bleeding issue indicative of a stomach ulcer or a symptom of inappropriate medication (Marieb \& Hoehn, 2007). Plasma $\mathrm{Hb}$ is a measure of the RBC content of the blood, which is indicative of blood volume and the ability to carry oxygen around the body, and in lower than normal amounts may contribute to symptoms of fatigue, dyspnoea (shortness of breath), and susceptibility to infection (Marieb \& Hoehn, 2007). Physical loss of blood due to bleeding from the fracture site will 
almost certainly reduce the $\mathrm{Hb}$ to a critical level, possibly necessitating a blood transfusion (Gwinnutt, 2008).

A review of the need for a blood transfusion was conducted by the Cochrane Collaboration (Carson, P, \& Hebert, 2012) and it was found that primarily transfusion was advised when the critical level of $\mathrm{Hb}$ was between 70 and $80 \mathrm{~g} / \mathrm{L}$ for older adult patients, but findings were not specifically associated with older hip fracture patients. Adunsky, Arad, Blumstein, Weitzman, and Mizrahi (2008), however, found that low $\mathrm{Hb}$ levels were not indicative of poorer outcomes with regards to immediate rehabilitation in hip fracture patients. They suggested that a blood transfusion only be administered when clinical signs of hypovolaemia, such as a low blood pressure (BP) or pronounced bleeding, were apparent. Foss and Kehlet (2006) also researched the need for blood transfusion in hip fracture patients and deduced that $\mathrm{Hb}$ needs to be checked on several occasions, both preoperatively and postoperatively, as there is difficulty in clinically detecting true loss of blood for a number of reasons. These reasons may include the continued loss of blood after the first admission $\mathrm{Hb}$ recording; intraoperative miscalculation of blood loss; continued bleeding into the tissues postoperatively when a drain is not insitu; and retention of fluid postoperatively, all of which may misconstrue the true level of $\mathrm{Hb}$ as blood volume may be high.

\section{The hip fracture event}

The hip fracture event will compound any comorbidities and prefall issues that the older adult patient has and heighten their awareness of being away from their normal domestic environment. This traumatic event will also be affected by how they can manage the surgical journey.

\section{Pathophysiology of osteoporosis}

The bones of the older adult are easily fractured because bone mineral density declines with age. In women, hormone changes accelerate this process and 
they will lose approximately $58 \%$ of their femoral bone density over their lifetime (Favus, 2006). The hormone oestrogen, which declines in production after about the fifth decade, and especially over the perimenopausal years, assists in the formation of osteoblasts (bone forming cells) and in the discouragement of osteoclast activity (break down of bone). Osteoclasts and osteoblasts normally work in tandem in the process of remodelling and rebuilding bone but in the absence of enough oestrogen the process is unbalanced and a net loss of bone density occurs (Favus, 2006). This process can also occur in men in later life and is assisted by a falling level of the hormone testosterone, which normally promotes bone formation. Oestrogen is also required for the utilisation of the mineral calcium, a necessity in bone formation. A reduced amount of oestrogen will discourage receptors in the intestine to absorb calcium, and will also encourage excretion of calcium from the kidneys via the urine (Favus, 2006).

An inadequate dietary intake of some vitamins and minerals can also affect the maintenance of bone density in the older adult. A less than optimal amount of calcium ( $\mathrm{Ca}$ ) and vitamin $\mathrm{D}$, which is required for bone mass and muscle strength and which is obtained from spending time in the sunlight, may result from a malabsorption issue, such as the inflammatory bowel condition Crohn's disease, but is also a symptom of renal (kidney) disease. Renal impairment reduces the activation of vitamin $\mathrm{D}$ and retention of $\mathrm{Ca}$, which may predispose to hyperparathyroidism. This can inhibit the utilisation of calcium (Ca) (Favus, 2006) leading to bone demineralisation. Deficits in Ca and vitamin D may also be caused by certain drugs, anorexia, and cachexia, which is a general muscle wasting process often seen in patients with a chronic disease process such as cancer (Favus, 2006). These changes in the body have the effect of increasing bone joint pain; soft tissue calcification, causing hardening of the heart valves and internal walls of the blood vessels; and also the heightened possibility of a fracture. (Marieb \& Hoehn, 2007) General reduction in optimal protein and calorific value in the diet may also stimulate bone reabsorption and excessive smoking and alcohol may encourage bone loss (Favus, 2006). 


\section{Pathophysiology of hip fracture}

Falling to the side and landing on the dominant part of the pelvic bone (the iliac crest) causes the angle of the neck of femur to be put under stress. This may cause the femur to crack, or break and dislodge. A fracture occurs more readily in the older adult patient because the reduction in bone porosity is unable to absorb the energy produced by the impact (Favus, 2006).

A hip fracture in the older adult patient occurs through the proximal shaft of the femoral bone, or through the head or neck of the femur. Types of hip fractures associated with this study (Figure 1) represent fractures to within $5 \mathrm{~cm}$ of the proximal femur and are also referred to as being associated with the greater trochanter or capsular region (Favus, 2006).
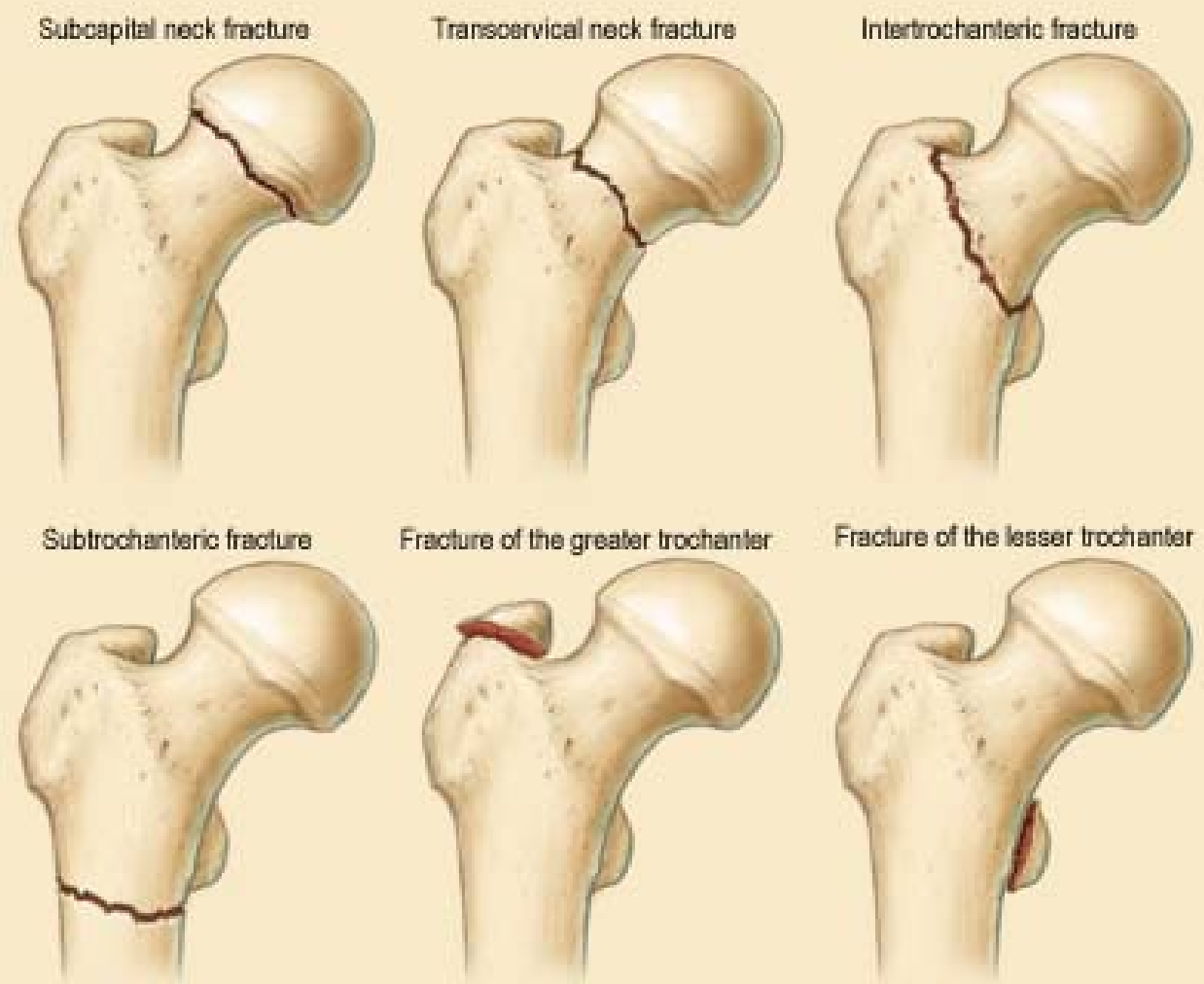

Figure 1: Hip fracture types and associated anatomy 
Clinical signs of a hip fracture injury are usually pain in the associated region, shortening of the leg, inability to weight bear on the affected side, and external rotation of the affected leg. The diagnosis is made by X-ray of the pelvic and upper leg region. The outer layers of bone (periosteum and cortex) may split at the fracture site and damage adjacent muscle, arteries or veins causing blood and plasma to escape into surrounding tissues. Bleeding may also occur directly from within the fractured bone if the fracture displacement is significant. This process will cause swelling into the leg and possible hypovolaemia (significant reduced circulating blood volume).

\section{Perioperative journey}

The older adult hip fracture patient is usually admitted to the accident and emergency department $(A+E)$ for initial assessment. A plan to manage this type of patient will be made as soon as possible so that an admission bed in the orthopaedic ward can be booked. Fast track initiatives, which are a pre-planned diagnosis and treatment regime, are common for hip fracture patients in hospitals worldwide, including in NZ, and are found to provide a more efficient and effective journey for the patient (Fergus, et al., 2011). The hip fracture patient is both an orthopaedic and geriatric admission, but will generally be admitted under the orthopaedic specialty. Assessment prior to surgery, however, needs to be by an orthopaedic surgeon, geriatrician, and due to the requirement for surgery, also an anaesthetist.

\section{Orthopaedic assessment}

The orthopaedic surgeon will examine the radiology report and decide on the appropriate treatment for the hip fracture. A full medical assessment will also be performed by the orthopaedic team and obvious medical issues flagged for treatment or further management. Assessment of the older adult patient involves evaluation of the main systems of the body, which are the cardiovascular, pulmonary and renal systems, along with the patient's social history, in order to 
plan effective care management during hospitalisation, provide for the best long term outcomes, and identify any existing comorbidities. Many older adult patients may be identified in their medical history as having cerebrovascular disease (CVD), peripheral vascular disease (PVD), congestive heart failure (CHF), chronic renal disease (CRD), dementia, or be diabetic.

Routine investigations of biochemistry parameters, such as $\mathrm{Hb}$, potassium $(\mathrm{K})$, sodium ( $\mathrm{Na}$ ), creatinine $(\mathrm{Cr})$, or glomerular filtration rate (GFR) along with urinalysis, and electrocardiogram (ECG) is recommended (Rai \& Mulley, 2007) to confirm or refute the presence of relevant degenerative disease. Further investigations should follow if irregular results are found; if there are symptoms of an unknown acute illness; and to remedy issues that will improve quality of life, such as identification of microbes on a slow to heal leg ulcer.

\section{Anaesthetic assessment}

The anaesthetic team will undertake a further medical assessment to check fitness for surgery, focusing especially on the issues of pulmonary, cardiac and renal function, circulatory volume, and further biochemistry parameters. They will also order further tests, if required, such as an echocardiogram (ECHO) to gain more information about cardiac function. The patient will need to be capable of coping with the rigours of surgery and either a general or regional anaesthetic. There will also be possible further loss of blood (hopefully minimal); metabolism of anaesthetic drugs through the kidneys; and reduced respiratory capability and cognitive function, due to the depressive effect of opioid or sedative medication administered during the anaesthetic and postoperative period. The anaesthetist will analyse assessment findings and formulate an anaesthetic plan. This plan will involve deciding on a suitable type of anaesthetic, either general or spinal; appropriate medication dosages; and a hydration management plan, which may primarily involve administration of intravenous (IV) $0.9 \%$ Normal Saline, Plasmalyte products similar in content to normal body fluid. Other appropriate fluid additives may be required to provide additional electrolytes, if indicated by 
biochemistry results, or to enhance blood content. Due to the fragility of the hip fracture patient assessing hydration requirements and administering appropriate fluids is a vital part of their perioperative management.

\section{Orthogeriatric assessment}

The purpose of the older adult patient assessment would be to judge both current medical and physical needs, as well as future and rehabilitation requirements. The older adult or geriatric specialist with a special interest in hip fracture patients is best advised to assess this type of patient as soon as possible after admission (Rai \& Mulley, 2007). Leung, et al. (2011) found in a retrospective study of 548 older adult hip fracture patients that those who had assessment by a geriatrician within 48 hours of admission, had a shorter wait time from admission to operation; shorter postop length of stay (LOS); half the one year mortality rate (11\% v 20\%); and better functional ADL status, compared with those who were primarily under orthopaedic management only. Fergus, et al. (2011) also found that provision for more frequent orthogeriatric assessment, within a specifically planned unit for older hip fracture patients, was the main element that shortened the period required in the acute care environment.

The path towards prefracture lifestyle is usually planned early in the care for the older adult with a hip fracture. An orthogeriatric specialist will assess whether the patient's cognitive, physical and medical needs will be best catered for by immediately returning to their usual residential care environment, or to a specialist centre in order to provide care that will optimise rehabilitation and mobility. Hospital treatment and care plans should be made to ensure the patient has optimal health and fitness to endure the rigors of rehabilitation physiotherapy to regain strength and function of the affected leg. Particular emphasis will be put on treating medical issues, such as antibiotics for pneumonia, providing a nutritious diet, and discussing patient preferences for post discharge care. Further discussions are advised to take place between hospital specialists, community doctors, and family members to ascertain the 
most appropriate ongoing support required for the individual patient (Rai \& Mulley, 2007).

Hip fracture patients who are younger and mostly independent before the hip fracture may be able to return promptly to their home environment if optimal effort is made to manage their care during the perioperative period in hospital (Rai \& Mulley, 2007). Older patients can anticipate lower functional postoperative activities of daily living (ADL) status but effective and efficient management of existing comorbidities and the perioperative journey will enhance these outcomes (Olsson, Karlsson, \& Ekman, 2007).

\section{Pain relief}

Pain is inevitable following a hip fracture, due to the fracture of bone and damage to surrounding soft tissues. It affects the morale of the patient, and possibly precipitates confusion (Rai \& Mulley, 2007). Pain relieving medication doses need to be carefully adjusted, because the older adult patient has less ability to process drugs, is more easily cognitively affected by opiates, tends to be more tolerant or uncomplaining of pain, and is possibly unable to communicate pain levels correctly or coherently (Stoelting \& Miller, 2007).

Pain level scoring by the older adult patient will require a level of cognitive thought which many may now be lacking. It may be particularly difficult to ascertain a pain score from patients with dementia, and from those who have difficulty speaking or understanding English or the caregiver's accent. Sieber, Mears, Lee, and Gottschalk (2011) concluded that opioid type drugs should not be withheld, however, from older patients due to concerns of overdose, as no significant relationship was found with patients who had delirium. It was found, however, that patients with diagnosed dementia did not receive as much opioid pain relief medication as patients who did not have dementia. This may have been because they could not voice their pain concerns. Regional nerve blocks have been recommended for this cohort of patients (New Zealand Guidelines 
Group, 2003) and Abou-Setta, et al. (2011) found nerve blocks to be effective in reducing the risk of delirium in the elderly hip fracture patient.

\section{Surgical repair}

The most common treatment for hip fracture is by permanent surgical fixation and is required if the patient is to have effective mobility and quality of life, even if they were primarily immobile prior to the incident. This procedure is undertaken under aseptic conditions in an operating theatre.

Surgical fixation involves securing the fractured bone in as near normal position as possible with screws, plates or other prosthesis (artificial replacement). The insertion of screws and plates involves drilling into the femur and head of femur, and insertion of metallic implants to stabilise the fracture area. Insertion of a partial prosthetic hip replacement involves removal of the fractured head of the femur at the neck level, and insertion of an artificial head and neck of femur. The prosthesis is anchored deep within the femoral shaft. Sometimes a full hip joint replacement is undertaken on the more active hip fracture patients. The surgery usually takes about $45-60$ minutes, but time in the operating theatre suite will be extended by administration of the anaesthetic and by recovery time.

Insertion of a prosthetic device is not only required to stabilise the hip fracture but to promote healing of the fracture site. The type of device will depend on the level and complexity of the fracture. Types of prosthetic device commonly used in New Zealand are chosen to match the extent of the hip fracture (Figure 2).

- Internal fixation with a proximal femoral nail (PFN) secures a fracture and/or displacement of the upper shaft of the femur.

- Compression hip screw (CHS) or CHS with plate are designed to stabilise fractures in the trochanteric region.

- Partial replacement of the head of the femur, with insertion of the stem into the upper femur, is done for high neck of femur (NOF) or capsular (head of femur) fractures. This latter procedure is called a 
hemiarthroplasty, and is usually done for fractures of the capsular region, because the blood supply has been disrupted to the head of the femur.

- Total hip joint replacement (THJR) is often performed on younger and more active hip fracture patients, which involves additional replacement of the acetabulum. This is done because a lesser prosthesis (hemiarthroplasty as described above) may not endure prolonged physical activity over a possible further twenty year life span of the patient.

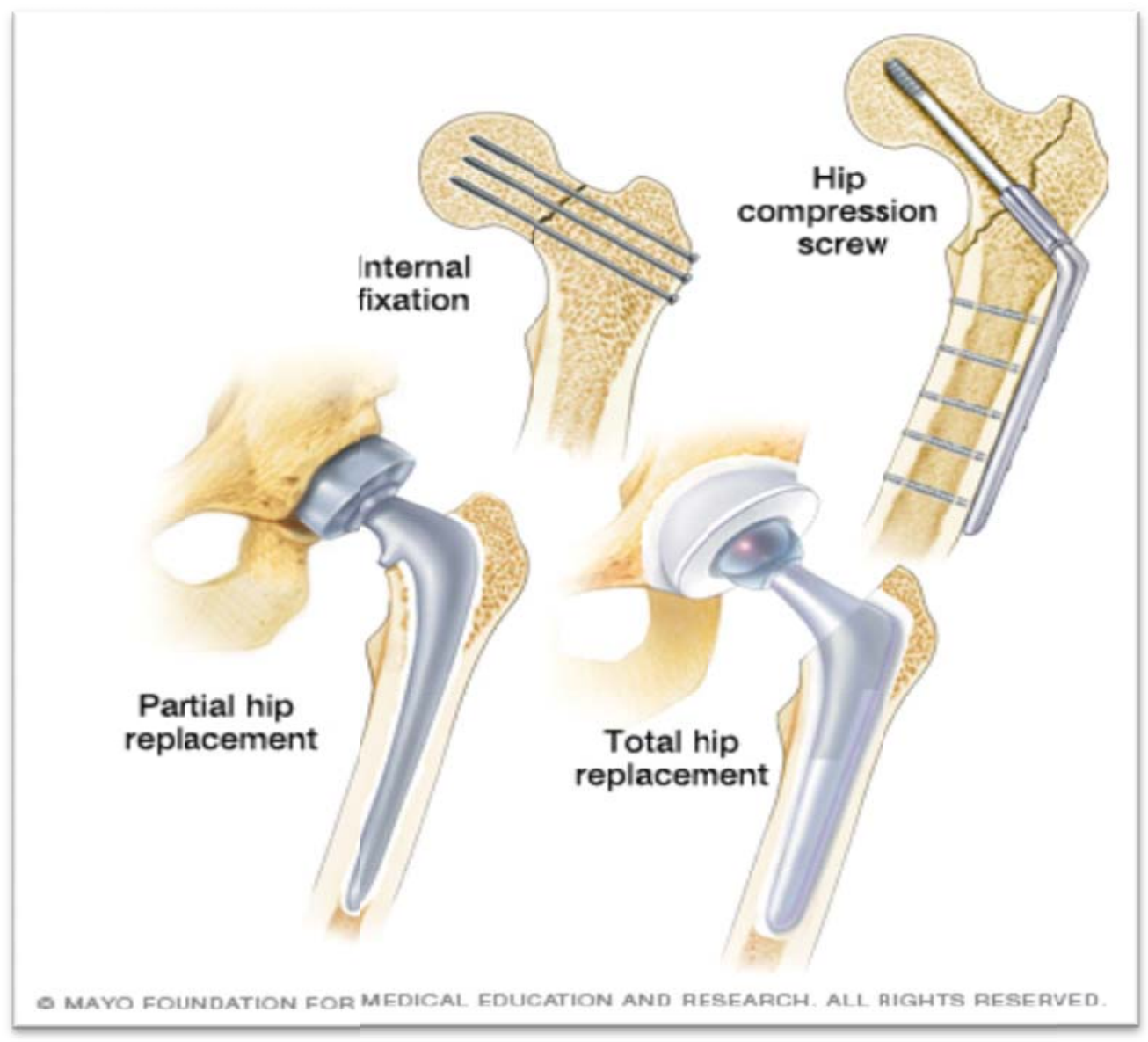

Figure 2: Hip fracture fixation types 


\section{Perioperative issues}

Further patient issues associated with the perioperative period are the likelihood of impaired renal function, a high likelihood of confusion, and possible lack of availability of appropriate food. Managing these issues is highly complex for the elderly hip fracture patient and requires in depth knowledge of their effects on the patient.

\section{Renal function}

The risks for kidney damage have been strongly associated with aging and hypertension, but primarily with having diabetes, and are further heightened by having cardiovascular disease (CVD) and atherosclerosis (Marieb \& Hoehn, 2007). Further risk factors include stress or trauma, such as a hip fracture event, or an acute kidney injury, such as severe toxicity from a drug or infection.

Renal function and a reduced ability to compensate for small changes in fluid volume in the older adult hip fracture patient is a critical issue with regard to the prevention of dehydration. If dehydration occurs the kidneys will not have the fluid quantity and pressure required (hypovolaemia) within the renal tubules to effectively process electrolytes and metabolites (waste products) such as creatinine or anaesthetic drugs. This will leave waste products of metabolism and an imbalance of electrolytes, as well as a possibility of inappropriately high doses of medications, within the intracellular and extracellular spaces causing medical issues such as metabolic acidosis. Metabolic acidosis (decrease in the $\mathrm{pH}$ value of the blood) will further affect the normal transfer and balance of electrolytes across the cell wall barrier (Gwinnutt, 2008) and occurs more readily in patients with a large degree of renal impairment. Metabolic acidosis can be fatal if left untreated as it causes depression of the neurological system and a possibly fatal coma (Marieb \& Hoehn, 2007). 
The effects of poor waste disposal of metabolites and regulation of electrolytes may produce symptoms of:

- Oedema due to an imbalance of sodium causing retention of water.

- Gastrointestinal symptoms of nausea and cramps due to accumulation of nitrogenous wastes.

- Confusion and lethargy due to metabolic acidosis.

- Acute haemorrhage and immune function reduction due to reduced cell production of platelets and leukocytes and caused by low hormone erythropoietin production in the kidneys.

- Pruritus (itchy skin) due to buildup of uric acid.

(Marieb \& Hoehn, 2007)

Renal impairment in association with a surgical procedure has the ability, therefore, to promote dehydration, thereby, worsening renal function and inappropriate medication uptake or excretion.

\section{Confusion}

Confusion (acute cognitive dysfunction), or delirium (confusion with hallucination), is common in the older adult patient and may be triggered by a range of factors such as infection; metabolic or nutritional disorders, for example anaemia or malnutrition; heart failure and hypoxia (low blood oxygen level); some cancers; trauma or surgery; head injury or brain lesion; and inappropriate drug dosage, opioids or analgesic agents (Longmore, et al., 2007; Rai \& Mulley, 2007). Pain and dehydration associated with many medical issues are, however, key causative factors in the incidence of confusion, and may simply be symptoms of a UTI (Stoelting \& Miller, 2007). The prevalence of new onset confusion in hip fracture patients has been reported as $20-28 \%$ (Duppils \& Wikblad, 2000; Faezah, et al., 2008). Confusion, therefore, can be a sign of an often multifactorial issue, not a symptom of one disease, and has been described as a syndrome (Rai \& Mulley, 2007). 
The signs of confusion in the older adult are those of an acute change in behavior which is often unusual and inappropriate. The confused patient may have disordered cognitive function, be delusional or disorientated, and not aware of their surroundings or reason for their admission (Rai \& Mulley, 2007). An unfamiliar environment is also very stressful for an older adult, especially if they have reduced cognitive function and are unable to remember what incident caused them to be admitted to hospital. The ageing process renders the older adult less able to manage challenging situations due to possible hearing or vision loss, causing distress and anxiety, and ultimately disorientation and confusion (Rai \& Mulley, 2007; Stoelting \& Miller, 2007).

The fundamental management of confusion in the perioperative period involves treating the source of the issue, and secondly assessing and providing appropriate hydration or fluid management (Rai \& Mulley, 2007).

\section{Malnutrition and fasting}

The older adult patient who has been admitted with a suspected hip fracture will be required to be fasted from food and fluids for a period of time prior to surgery. This is a traditional practice and commonly known as nil by mouth (NBM) in the medical environment. When a patient requires surgery of any type the stomach needs to be empty in preparation for a possible general anaesthetic as there may be the risk of regurgitation of stomach contents and subsequent inhalation causing a blockage of the bronchus (Gwinnutt, 2008) and prevention of adequate oxygenation via the lungs to the circulatory system.

The requirement to fast preoperatively renders the patient unable to consume regular fluids if they are thirsty. This requirement could exacerbate a possible existing dehydration. Mentes (2006) found this was common in one study, with $31 \%$ of older adults in residential care, being dehydrated at least once over a six month period. Fluid replacement will, therefore, be required to be assessed, and 
initiated intravenously via a small cannula, usually inserted in a vein of the forearm. Providing small amounts of clear fluids orally on a regular basis during this often unpredictable wait time to surgery could prevent this problem. Power, et al. (2012) studied the effect of reduced NBM, or fasting, times on adult surgical patients and deduced that there was less reported preoperative thirst, headache and nausea in the group allowed clear fluids up to two hours preoperatively and food up to six hours preoperatively. Issues with intraoperative aspirations were also not apparent. It has also been found that approximately $25 \mathrm{ml}$ of gastric fluid left in the stomach prior to surgery has no postoperative complications, and this is achieved with a variety of long (from midnight) and short (60 minutes preoperatively) fasting times with unrestricted clear fluids (Brady, Kinn, Stuart, \& Ness, 2003). The Royal College of Nursing (2005) supports this in their clinical practice guidelines Perioperative fasting in adults and children and recommends $30 \mathrm{ml}$ of clear fluids are allowable less than 2 hours preoperatively, if required, even with high risk patients.

The older adult hip fracture patient may also be malnourished on admission as confirmed by a NZ study where $42 \%$ (Hanger, et al., 1999), and in a UK study $56 \%$ (Nematy, et al., 2006) of hip fracture patients were diagnosed as such on admission. Due to the requirement to be fasted from food preoperatively these patients may develop a worsening state of malnutrition over this period. Caccialanza, et al. (2010) demonstrated that those patients who had a moderate to severe malnutrition score on admission were $37 \%$ more likely to have a prolonged hospital stay, a possible worsening malnourished state, and have a $5 \%$ risk of further weight loss occurring if hospital stay was longer than three days. The malnutrition score used a formula based on the patient's height, weight, body mass index (BMI) and serum albumin on admission (Caccialanza, et al., 2010). This study looked at normally ambulatory adult patients, admitted into either a surgical or medical ward, with average age of 60 years. A cohort of older adult patients would be expected to fair much worse. 
Protein in the diet is predominantly required to rebuild cells, especially in the skin and bones, and maintain muscle strength. Albumin is a protein produced in the liver and is required to assist in the transport of some electrolytes, hormones and medications in the blood, and maintain water pressure within the blood (Marieb \& Hoehn, 2007). It is a blood biochemistry indicator of protein availability and in lower than normal levels may be associated with malnutrition, malabsorption in the gut, such as in ulcerative colitis, nephrotic syndrome (liver disease), or simply stress or trauma (Hershkovitz, Kalandariov, Hermush, Weiss, \& Brill, 2007; Rai \& Mulley, 2007). A low albumin level on admission has been used as an indicator of malnutrition in the hip fracture patient in a number of studies, where Nematy, et al. (2006) showed that there was further decline in malnutrition levels in older adult patients while in hospital, and Hershkovitz, et al. (2007) showed that a shorter length of hospital stay and improved cognition had a relationship with higher albumin levels. Plasma albumin may also be low due to excess protein being excreted by the kidneys in renal disease (Marieb \& Hoehn, 2007).

Limitation of the hip fracture patient's fasting, or NBM period, and offers of appropriate and nutritional fluids during the preoperative period could reduce the risk of malnutrition, further renal impairment and issues of confusion.

\section{Fluid management and fluid balance}

Fluid management is the process of providing appropriate hydration or fluids for a person's bodily requirements. Fluid requirements are normally regulated according to the body's physiological needs and how much the kidney excretes in urine, and is adjusted by an automated mechanism called osmolality of electrolytes (Marieb \& Hoehn, 2007). People who are fully independent are capable of providing their own fluid requirements by freely accessing drinks from a variety of sources, but hip fracture patients who are immobile and in hospital may not. Patients in hospital have fluids provided by offers of drink, IV or subcutaneous infiltration, or from within solid food. Inadequate fluid 
management can lead to dehydration, hypovolaemia and hypervolaemia, seriously affecting all bodily functions.

\section{Hydration}

Effective hydration, or circulating fluid within the body, ensures that tissues are adequately provided with available electrolytes and have waste products of metabolism removed, for optimal functioning of the body. Body fluids make up approximately $60 \%$ of an adult's body weight (approximately 40 litres in a $70 \mathrm{~kg}$ adult), and assist in the transport of electrolytes and metabolites between the intracellular and extracellular spaces (Gwinnutt, 2008). Intracellular fluid is contained within the cells of the body, and blood plasma (within the blood vessels) and interstitial (around tissue) fluid make up the extracellular system (Marieb \& Hoehn, 2007). The fluid is primarily water, is supplied to the body by drinking and eating, and is lost through urine excreted by the kidneys, faeces, and insensible loss through the skin, mucous membranes and lungs while breathing (Gwinnutt, 2008).

\section{Assessment of hydration status}

Measuring hydration status to ensure adequacy for an individual patient is not possible with one simple test and, after taking several different types of measurements, normal parameters are established over time for individual patient assessment (Ward, 2011). Physical signs and observations, a variety of easily used assessment options and analysis, and biochemistry reports are all required to assess hydration status.

\section{Assessment options}

Commonly used assessments to gauge hydration status are made utilising parameters such as urine volume output (Zeitz \& McCutcheon, 2006), urine specific gravity (USG) (Wakefield, Mentes, Diggelmann, \& Culp, 2002), urine colour (Mentes, 2006), external jugular vein pressure (JVP) palpation (Sankoff \& 
Zidulka, 2008), assessment of dampness of oral mucosa, tissue turgor, and palpable and visible peripheral perfusion (Rai \& Mulley, 2007; Stoelting \& Miller, 2007). Studies have not been found that systematically address the issue of hydration status, but review of two appropriate studies with regards hip fracture patients, undertaken by the Cochrane Collaboration, found that invasive monitoring of hydration status intraoperatively using a central venous pressure (CVP) monitor, increased administration of fluid volumes and reduced total length of hospital stay (Price, Sear, \& Venn, 2004). No studies were found in this review to utilise invasive monitoring of hydration status pre- or postoperatively under normal acute care circumstances.

\section{Physical signs}

External signs of dehydration may be apparent if the patient has reduced skin turgor (elasticity), is thirsty, or has dry oral mucous membranes. Skin turgor is, however, difficult to assess accurately in the older adult, as much of their skin already has reduced elasticity, but tighter areas across the sternum will demonstrate this. Complaining of thirst is an obvious sign of dehydration in the older adult patient as their thirst mechanism is reduced and they are generally undemanding of drinks. This may be due to being unable to drink because they have swallowing issues, not wanting to drink in case they are incontinent, and forgetting to drink due to cognitive decline (Mentes, 2006). A visibly dry mouth is also consistent with lack of fluids as body fluid is prioritised to the vital organs and there is reduced flow to the peripheral tissues (Marieb \& Hoehn, 2007).

\section{Vital Observations}

Fundamental vital observations for hydration status include recordings of blood pressure (BP), pulse, respirations and temperature. People resident in an older adult facility may or may not have daily monitoring of these observations but patients who are unwell will require more regular recordings. Timing of these recordings should be individual to the patient but is usually done on a four hourly time pattern (Zeitz \& McCutcheon, 2006). 
Those patients at risk of medical complications or worsening of their comorbidities can be more often observed if vital observations suggest this. The Patient at Risk (PAR) scoring system in use at CCDHB (Capital and Coast District Health Board, 2008) is activated at the following levels for systolic BP (BPsys) $<101-179 \mathrm{~mm} \mathrm{Hg}>$, pulse $<51-100 \mathrm{~min}>$, and respirations $<9-14$ min>. Systolic BP is of importance in this situation as it is an indicator of the maximum arterial pressure that cardiac output can produce (Shiel \& Stoppler, 2008). A lowered BPsys on standing, known as postural hypotension, is also an indicator of dehydration, but difficult to perform on a bedridden patient. A diagnosis of possible dehydration would be indicated by a combination of lowered BPsys (due to hypovolaemia), and a raised pulse and respirations, attempting to compensate for the hypovolaemia.

Body temperature, normally around $37^{\circ} \mathrm{C}$, is of concern when it is increasing or decreasing over the day from baseline measurements, but may normally fluctuate between $36^{\circ} \mathrm{C}$ and $37.2^{\circ} \mathrm{C}$ for individual patients (Shiel \& Stoppler, 2008). An elevated temperature may indicate an infection is present which may assist assessment towards a preadmission medical issue.

\section{Body water}

Total body water (TBW) of an individual can also be utilised as a calculation of body water loss or hydration status. TBW reduces with age and weight loss, as there is very little water in fat and relatively more so in muscle (Marieb \& Hoehn, 2007). If an older adult has not been drinking adequately for several days their body weight will have significantly reduced, and this is reflected in their TBW. Unfortunately this is only useful if initial weight is known and the patient can be easily weighed. It is beneficial to know, however, that a reduced water intake of one litre per day for an elderly $44.5 \mathrm{~kg}$ woman would reduce her TBW by $5 \%$, which would either reduce her extracellular fluid (ECF) by $15 \%$ or plasma volume by nearly $60 \%$ (Wotton, Crannitch, \& Munt, 2008). Bioimpedence assessment 
(which measures refilling speed in peripheral veins) and analysis can be used to ascertain the percentages of extra- and intracellular fluid and TBW, and in conjunction with body mass index (BMI), have been successfully used to measure levels of dehydration (Mentes, 2006).

\section{Urinalysis}

Evaluation of urine can be a very clear indicator of dehydration. Undertaking a urinalysis will give evidence of excreted matter in the urine, showing evidence of abnormal function of systems of the body, for example renal or endocrine.

A routine urinalysis should show evidence of normally found crystals and casts (deposits), a pH of $4.5-8.0$, be pale yellow in colour, and have a pleasant mild odour (Marieb \& Hoehn, 2007). Specifically designed dipsticks are widely available to measure $\mathrm{pH}$, and colour charts have been successfully used to monitor slight changes in urine to indicate hydration status in nursing home residents (Mentes \& Culp, 2003). Measures of USG, utilising a specifically designed meter placed in the urine, can also indicate worsening dehydration. This will show increasingly concentrated urine, due to the increased proportion of casts (Mentes \& Culp, 2003). Some hormones may be present in small amounts, but the presence of glucose, protein or leucocytes (measureable on a commercially available dipstick), is abnormal and may indicate a range of conditions including renal failure, uncontrolled diabetes, or infection (Longmore, et al., 2007; Martini \& Welch, 2006).

Urine volume is a clear indication of how well the kidneys are functioning and a state of dehydration will reduce the amount of water the kidneys have to filter. Routine observations of urine output of less than $0.5 \mathrm{ml} / \mathrm{kg} / \mathrm{hr}$ is at the lowest point of acceptability but $50-100 \mathrm{ml} / \mathrm{hr}$ is best for organ perfusion (Gwinnutt, 2008). The difference between oral, IV and solid food fluid intake, and urine volume output is a measure of the allowed deficit in urine output to fluid intake. This deficit, which will vary between individuals, takes into consideration the fluid 
lost in faeces, and insensible loss through the skin, mucous membranes and lungs. In order to maintain normal kidney function a healthy adult needs to excrete approximately one litre of fluid per day. Patients with a degree of renal impairment, however, will either have reduced weak urine or if kidney function improves and fluid intake is high may have weak polyuria (excess excretion of urine) due to their inability to concentrate (produce) urine (Marieb \& Hoehn, 2007).

\section{Biochemistry indicators}

Plasma biochemistry results, obtainable from a variety of body fluid samples, indicate how well the body is functioning, and can indicate hydration status by measuring levels of electrolytes and metabolites, and other haematological elements of the blood. Plasma osmolality may also be monitored to show improvement or deterioration in hydration status, by measuring quantities of the solute (dissolvable) particles in the blood which are required for normal body function, such as the electrolyte sodium ( $\mathrm{Na}$ ). Electrolytes are soluble materials that require a finely balanced mechanism of fluid transport between the cellular spaces of the body. If some electrolytes are present in greater or lesser amounts than usual, such as $\mathrm{Na}$ and potassium $(\mathrm{K})$ and they become unbalanced, medical conditions such as dehydration may occur (Martini \& Welch, 2006). Products of metabolism (waste products of energetic cell breakdown) are primary indicators of effective function of the systems of the body, and are commonly seen in monitoring the renal system and noting certain blood plasma components, such as creatinine $(\mathrm{Cr})$, urea, albumin, and the glomerular filtration rate (GFR).

Sodium is required to maintain the appropriate balance of water between the intracellular and extracellular spaces and is provided to the body by normal eating and drinking. Sodium attracts water so when the body is dehydrated there will be a higher than normal concentration of sodium in the cells and water will be transported out of the blood vessels into surrounding tissues to lessen the 
concentration. This may cause a loss of circulating fluid (hypovolaemia), and effect a possible lowering in blood pressure, or hypotension. Worsening dehydration may result in dry skin with less turgor, confusion, seizures and muscle weakness (Longmore, et al., 2007). Plasma Na levels that are either elevated or lower than normal may be indicative of possible dehydration (Rai \& Mulley, 2007).

Potassium $(\mathrm{K})$ is an electrolyte that is required for normal functioning of muscle and is relevant in low or raised amounts. High levels of $\mathrm{K}$ may affect cardiac muscle rhythm causing arrhythmia, ineffective heart function and poor perfusion of vital organs (Longmore, et al., 2007), and neuromuscular problems, affecting breathing and the respiratory system. Muscle weakness is a sign of lowered plasma $\mathrm{K}$. Large amounts of $\mathrm{K}$ can be easily lost by individuals taking diuretic (water reducing) medication, causing water to be removed quickly from the body with less time in the filtration and reabsorption mechanism of the kidneys. Electrolytes such as K may, therefore, be excreted inappropriately. Potassium is normally provided to the body through a nutritional diet and the excess amount is excreted via the kidneys.

Creatinine $(\mathrm{Cr})$ is a by-product of the metabolism of muscle and is an indicator of how well the kidneys are functioning when measured in blood biochemistry. The GFR also demonstrates this by providing information about how well the tiny glomerular of the kidney are filtering fluids and solutes such as $\mathrm{Cr}$ (Longmore, et al., 2007), and can be indicative of a degree of renal failure (Table 1). A raised plasma $\mathrm{Cr}$ is usually apparent alongside a reduction in GFR, but an elevated plasma $\mathrm{Cr}$ may not be evident, however, until there is at least a $50 \%$ reduction in renal function (Stoelting \& Miller, 2007). 
Table 1

Stages of Renal Disease

\begin{tabular}{|llc|}
\hline Stage & Description & $\begin{array}{c}\text { GFR } \\
\text { mL/min/1.73 } \mathbf{~ m}^{\mathbf{2}}\end{array}$ \\
\hline 1 & $\begin{array}{c}\text { Slight kidney damage with normal or } \\
\text { increased filtration }\end{array}$ & $>90$ \\
& Mild decrease in kidney function & 60 to 89 \\
2 & Moderate decrease in kidney function & 30 to 59 \\
3 & Severe decrease in kidney function & 15 to 29 \\
4 & Kidney failure requiring dialysis & $<15$ \\
5 &
\end{tabular}

(Gwinnutt, 2008)

\section{Dehydration}

Dehydration is a state of having a less than optimal amount of fluid in the intraand extracellular compartments of the body to provide effective hydration and is caused by water depletion. Dehydration primarily affects the cardiovascular and renal systems, but also affects cognitive function and peripheral perfusion (blood circulation to the hands and feet) (Longmore, et al., 2007). Loss of fluid in the cardiovascular system may be caused by hypovolaemia, due to bleeding; a metabolic imbalance (described in the previous section); vasodilation (peripheral dilation of blood vessels), due to effects of drugs or other external stimulation such as temperature; or a third space shift of fluid due to tissue trauma (Gwinnutt, 2008). Third space shift is explained by the transfer of fluids from within cells to within spaces in the body, such as the abdominal cavity. This fluid is therefore lost to normal circulation.

The renal system produces urine by maintaining a concurrent mechanism of filtration of body fluid through the renal tubules and glomerular, with a good renal blood supply required for optimal function (Marieb \& Hoehn, 2007) (Figure 3). 
Dehydration may prevent this mechanism from effectively excreting waste products, and also cause long term damage, due to limited perfusion of the kidney tissue causing necrosis (death of tissue) of the fine glomerular tissue.

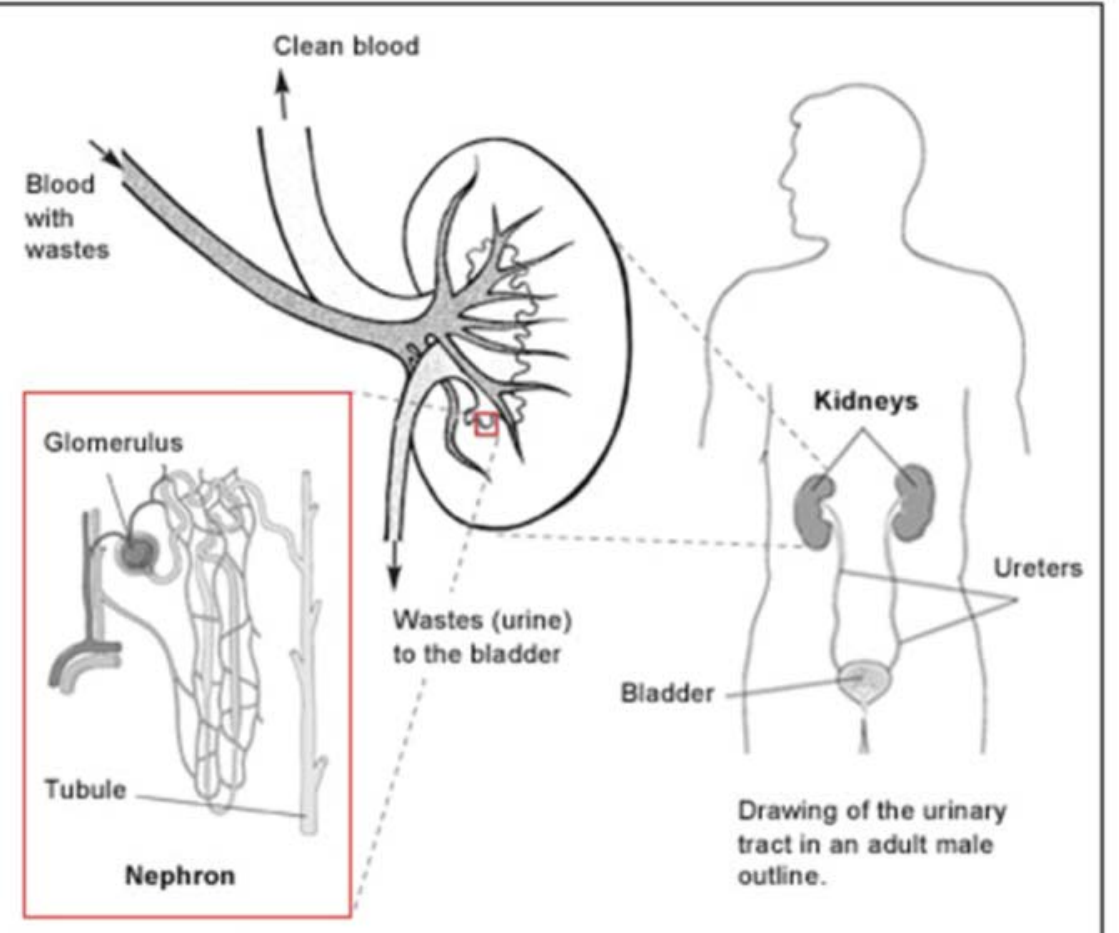

Labels (above) show where blood with wastes enters the kidney. clean blood leaves the kidney, and wastes (urine) are sent to the bladder. An inset (lower left in red box) shows a microscopic

view of a nephron. Labels point to the glomerulus and the tubule.

Figure 3: Renal function

There are many symptoms of possible dehydration and some categorical signs.

Symptoms of dehydration may include thirst, having dry mucous membranes, such as in the mouth, cool extremities, tachycardia or hypotension, and dyspnoea. Signs of dehydration may include lower than normal and more concentrated urine output, usually less than $0.5 \mathrm{ml} / \mathrm{kg} / \mathrm{hr}$ (Gwinnutt, 2008); raised plasma urea and $\mathrm{Cr}$ (both products of metabolism) and $\mathrm{Na}$; and an increasing metabolic acidosis. Dehydration has a profound effect on the renal and cognitive 
systems in the older adult patient (Rai \& Mulley, 2007) and may be heightened by existing dietary issues.

\section{Hypovolaemia}

Hypovolaemia is measured by a lower than normal BP and is often accompanied by a weak pulse that is unable to be palpated at a peripheral artery. Causes of hypovolaemia may include physical blood loss from the vascular system into surrounding tissues, due to haemorrhage from a damaged organ, tissue, or bone; vasodilation due to a medication reaction; and an inadequate intake of either oral or intravenous fluids, and prolonged vomiting or diarrhoea.

Hypovolaemia may cause hypotension; inadequate perfusion of the brain causing confusion, or delirium; ineffective functioning of the kidneys and liver; and less oxygen perfusion to all tissues of the body. The effect on the body may also be poor metabolism of medication, fainting, fatigue, dehydration, metabolic acidosis, or hypoxia (Gwinnutt, 2008).

\section{Hypervolaemia}

Hypervolaemia is defined as retention or an excess amount of circulating blood volume. Poor functioning of the renal filtration system, causing retention of some electrolytes such as $\mathrm{Na}$ and $\mathrm{K}$, will assist in this fluid volume retention and may cause overload of other vital areas of the body, causing stress on the heart, kidneys and pulmonary system. This may also be exacerbated by administration of inappropriately large amounts of IV fluids causing oedema (swelling of the tissues), especially around the lungs and hearts, precipitating breathlessness and congestive heart failure (Longmore, et al., 2007). Incorrect dosage of diuretic medication, while normally promoting fluid excretion, may instead also encourage retention of fluid. 


\section{Fluid replacement}

Careful fluid management in the acutely unwell patient will reduce the risk of associated issues with regards hypovolaemia and hypervolaemia. Fluid replacement associated with the preoperative, intraoperative and postoperative patient journey (combined period known as perioperative) may be required for fluid loss due to fasting, sweating due to fever, haemorrhage, diarrhoea, vomiting, third space shift, or a complex medical issue, such as fluid weeping from burns, in addition to normal physiological requirements.

Calculating quantities for fluid replacement, either oral or IV, in individual patients is difficult and requires a detailed knowledge of the patient's comorbidities to assess medical needs (Gwinnutt, 2008). Calculating fluid requirements in the completely dependent patient is also time consuming and often inaccurate due to lack of historical knowledge around fluid intake patterns. Assessing the patient's state of hydration will involve knowledge and analysis of some or all of the indicators of hydration status (as mentioned previously), plus knowledge of immediate elements, such as length of time fasted while waiting for theatre, and anticipated blood loss during surgery (Gwinnutt, 2008).

Replacement fluids for the acutely unwell patient is usually given via direct IV access and involves calculation of estimates of recent fluid intake deficit, analysis of hydration status (as previously discussed), current medical history, and arterial blood gases (Stoelting \& Miller, 2007). Fluid quantities are calculated on a weight by volume per hour rate that the patient has not had their usual amount of fluids and replaced accordingly (Stoelting \& Miller, 2007), but need to carefully adjusted for patients with conditions such as CRD, who may have limited fluid tolerance. Appropriate types of volume enhances will be chosen depending upon further intra- or postoperatively fluid loss. 
Types of suitable oral and IV fluid replacements need to be assessed, depending on individual physical attributes, such as being bedridden or having dysphagia (difficulty swallowing), or whether the patient has a low level of an essential electrolyte, such as K. Generally no food intake is allowed prior to surgery but allowable fluids may involve some solid components, such as in tea, milo or soup. This is limited to clear liquids, such as water or broth, nearer to operation time. If patients are not able to consume enough fluids, due to immobility or illness, IV fluids will be commenced to prevent dehydration. Types of IV fluids will be similar to plasma in $\mathrm{pH}$ and composition, containing some electrolytes, such as $\mathrm{Na}, \mathrm{K}$, and $\mathrm{Ca}$, in order to replace fluid volume within the extracellular and intravascular spaces and provide for depleting electrolytes. If fluid volume is required for improving intravascular volume only a more dense fluid with a high molecular weight (less osmolality), will be chosen, such as Haemacel. This will provide specific amounts of required electrolytes that the patient may be lacking, such as $\mathrm{Na}$ or $\mathrm{Ca}$ (Gwinnutt, 2008). Blood products may also be required, such as red blood cells (RBC) for extended bleeding, and fresh frozen plasma for clotting issues (Stoelting \& Miller, 2007).

Assessing and managing fluid requirements is difficult with a person of any age, but particularly relevant to the older adult patient who may have a history of comorbidities that cause complex medical issues.

\section{Current guidelines for treatment}

Current management and treatment of the older hip fracture patients is guided by traditional routines for acute orthopaedic patient admissions and in many institutions optimised with a specific care plan (Fergus, et al., 2011; Miura, DiPiero, \& Homer, 2009; Montalvo, et al., 2011). Available guidelines in NZ for this cohort of patients (New Zealand Guidelines Group, 2003) suggest that this is preferable. 
Recommendations produced by the New Zealand Guidelines Group (NZGG) are an evidence based summary for the management of hip fracture patients in New Zealand (New Zealand Guidelines Group, 2003). The summary is based on national and international research and recommendations with the aim to assist specialists and policy makers manage the older adult patient admitted with hip fracture, during the acute and immediate post-acute phase, in the most effective manner. Recommendations were made by a multidisciplinary group of health professionals.

The 2003 NZGG best practice evidence based guidelines - Acute management and immediate rehabilitation after hip fracture amongst people aged 65 years and over (New Zealand Guidelines Group, 2003) - are as relevant today as they were ten years ago, and are supported in the main by current research. Key recommendations within these guidelines appropriate to the perioperative management of the older adult hip fracture patient and relevant to this study are defined broadly and listed below.

- Formal fast track protocol for admission of hip fracture patients should be undertaken in order to reduce delays to diagnosis, medical and surgical assessment, ward admission, and early treatment for existing comorbidities.

- Careful fluid management to reduce risk of dehydration, overload, or electrolyte imbalance.

- Leg traction is not necessary as it does not significantly reduce the need for analgesia while waiting for surgery.

- Pain assessment and medication tools should be objective and methodical to ensure patients who are uncomplaining or have dementia get adequate 
pain relief medication. A variety of administration routes need to be considered, including nerve blocks, and minimal use of opioids, to reduce the risk of confusion, respiratory depression and adverse drug side effects.

- Oxygen should be administered proactively to improve tissue perfusion.

- Nutritional supplements need to be administered to reduce mortality and morbidity.

- Catheterisation is not routinely preferred, as it may delay return to normal voiding.

- Delirium and dementia management should be proactive and include formal measures of cognitive function on admission.

- Admission to surgery time should be minimised and ideally less than 24 hour to reduce total length of stay in hospital and adverse outcomes of morbidity and mortality.

- Regional anaesthesia has correlations with less postoperative deep vein thrombosis but there is little difference in mortality rates with general anaesthesia.

- Early rehabilitation planning that involves assessment by a multidisciplinary team (MDT) of health professionals, including a specialist geriatric consultant familiar with orthopaedic issues, in order to reduce length of stay and to optimise pre fracture mobility and activity levels. (New Zealand Guidelines Group, 2003) 
The issues within these recommendations will be utilised in post data collection discussion for his study.

\section{Overall clinical view}

This study was initiated following a review of literature regarding fluid management in older adult hip fracture patients (Ward, 2011). It was found that:

- Patients were more likely to be malnourished or dehydrated on admission.

- Due to multiple comorbidities, including renal impairment, and fluids being withheld or given as replacement, disruption of the patient's electrolyte balance can occur.

- Nurses, as their primary care-givers, need to be aware of any deviations towards dehydration.

- Assessing fluid requirements is not a straightforward task.

- Hydration status is of particular importance as the patient needs to have the ability to process anaesthetic drugs, and generally cope with the rigors of surgery.

Comorbidities are numerous in this age group and include renal impairment, cardiac issues, vascular disease, chronic lung conditions, and DM. Many also have some form of dementia, metabolic disease, such as hypothyroidism, or neurological condition, such as Parkinson's disease. Their domestic situation ranges from living independently alone at home to living in hospital level or specialist care unit, and independent to completely dependent on healthcare personnel or family members for all their ADLs. 
When a patient presents in hospital with a fragility hip fracture it will be as a result of a fall and be associated with several confounding issues. There may have been a delay before the patient was admitted due to being alone and undiscovered, or the fall not being reported. The patient will also be immobile, due to the pain and the fracture not permitting weight bearing activities and they may have missed a meal prior to admission and then required to be NBM. The older adult patient may be malnourished and possibly anaemic, will be out of their normal environment, and may have an underlying infection or other medical condition that is unrelated to the fall, such as a UTI or pneumonia. The patient will also have a degree of renal impairment, and have a high chance of having some onset of dementia or lowered cognition. Finally they may already be confused or delirious as a result of the stress of the admission, dehydration, an infection, electrolyte imbalance, or pain.

Statistics associated with this group of patients attempt to explain the extent of the concerns within this population mass in NZ and are itemised below (Table 2):

\section{Table 2}

NZ hip fracture patient statistics

- $13 \%$ - older adult population $65 \mathrm{yr}+$

- $25 \%$ - older adult population by 2030

- $43 \%$ - falls require hospital admission annually

- $82 \%$ - falls related admission $75 \mathrm{yr}+$

- 3000+ - hip fracture admission nationally

- 200 - hip fracture admission annually CCDHB

- 83yrs - average age

- $75 \%$ - women

Mortality rates are reported after hip fracture to range from $10 \%$ after one month, $12 \%$ after six months, and $20-30 \%$ after one year (National Clinical Guideline 
Centre, 2011; Thwaites, et al., 2007). Morbidity rates are closely aligned as reduced post hip fracture mobility (Thwaites, et al., 2007) takes its toll on general health and existing comorbidities. This simple hip fracture admission has become a multiple issue patient who needs to have a major operation, and will need multidisciplinary rehabilitation after surgery.

The older adult is susceptible to fractures due to reduced bone density (osteoporosis) and physical frailty. Osteoporosis is common in the older adult as the hormone oestrogen reduces over time in both men and women but has a more pronounced effect in women. Oestrogen is required for the maintenance of bone growth and the retention of calcium, and a poor diet and health, with less than optimal protein intake, along with alcohol and smoking, will also hasten this process.

Osteoporosis in the older adult age group has been acknowledged by agencies worldwide (Bone and Joint Decade, 2012; New Zealand Orthopaedic Association, 2003) and guidelines and reports outlining essential care for this cohort of rapidly increasing numbers, are paralleled across nations, such as in the UK (National Clinical Guideline Centre, 2011), and in NZ (New Zealand Guidelines Group, 2003). Research indicates that some issues highlighted in these guidelines do make a difference, namely reduction in delirium, fast tracking of patients through the initial hospital admission phase, reduced urinary catheter requirements, early involvement of an orthogeriatrician, focus on nutritional requirements, appropriate pain management, and reduced time to operation.

Best practice for hydration assessment, monitoring and management of the older adult hip fracture patient has been reported as inconclusive in a Cochrane Collaboration review on fluid optimisation following proximal femoral fracture (hip fracture) (Price, et al., 2004) and further research on the topic is difficult to find. A further Cochrane Collaboration report on perioperative fasting of adult patients to prevent perioperative complications was first published by Brady, et al. (2003) 
and reviewed in $\mathbf{2 0 1 0}$ with no change to conclusions. The latter reported that more research is required for those patients considered at risk, such as the older adult; that the at risk patients researched had no worse outcomes with a lesser NBM time; and that partaking of clear fluids up to two hours preoperatively was just as appropriate now as it was when the report was first published.

Reducing preoperative fasting requirements is, however, a key issue for the older adult patient and instigation of regular offers of allowed fluids is paramount. Offering small amounts of appropriate clear fluids to the acutely immobile hip fracture patient may provide comfort for an unrecognised thirst, assist drinking for the dysphasic or physically weak patient, and provide the arm that cannot pour the water jug or reach the glass on the side table.

The preoperative fluids, therefore, need to be managed carefully alongside the general care of this complex patient, and the patient needs to be well hydrated and with a reasonable balance of electrolytes to assist cardiac and renal function. This is especially required in order to manage the added stress of surgery, which includes the metabolism and excretion of anaesthetic drugs, further blood loss, and opioids for pain relief. Assessment of comorbidities, current medications, alongside hydration status is also paramount. The effect of the requirement to fast, which limits food and normal drinks, is also exacerbated by the patient's immobility, frailty and lethargy.

Dehydration is easily attained and very quickly affects renal and cardiac function, causing retention of waste products of metabolism, and leading to possible acidosis. Blood pressure regulation may also be affected, leading to hypo- or hypertension, poor peripheral circulation, and instigating a possible shutdown of vital organs. Fluid replacement requirements are usually gauged according to assessment of hydration parameters and vital baseline observations by house surgeons, registrars and anaesthetists, but are based on historical results over a 24 hour period. 


\section{Purpose of further research}

This study aims to achieve locally obtained fluid management data applicable to the initial acute care of the hip fracture patient in NZ; findings that relate to short term outcomes of quality and effective care; and acknowledgement of issues in this orthogeriatric cohort with issues of extensive comorbidities, nutrition, hydration, and pain management, waiting time to surgery, and increasing age and fragility. Local research in NZ has been undertaken with older adult hip fracture patients over the last ten years, but has focused on mortality and morbidity rates, and rehabilitation issues (Davison, et al., 2001; Thwaites, et al., 2007; Young, et al., 2006). The journey of the acute orthopaedic older adult patient needs to be more efficient and effective, and the NZ Orthopaedic Association support of the NZ Bone and Joint initiative program in NZ (Bossley \& Miles, 2009) needs to be encouraged. Current documents from within CCDHB and the wider NZ medical environment associated with this journey are outlined below.

Patients on the hip fracture journey are governed by the following criteria at CCDHB:

- Fast track protocol through the accident and emergency department to ensure that the patient is settled into a ward environment as soon as possible (Appendix D).

- Perioperative fasting of adult patients policy (Capital and Coast District Health Board, 2011), updated to current best practice just under a year ago, recommends clear fluids available up to 2 hours preoperatively and, if required within 2 hours of surgery, $30 \mathrm{ml}$ of clear fluids at a time (Appendix B).

- Traditional baseline observation monitoring documented on the Adult Vital Signs Chart (AVSC) of temperature, pulse, respirations, BP, urine output, and level of consciousness (Appendix E).

- Acute management and immediate rehabilitation after hip fracture amongst people aged 65 years and over (New Zealand Guidelines Group, 2003), 
which provides background guidance for the MDT of surgeons, geriatricians, nurses and allied health professionals (Appendix A).

The burden of musculoskeletal injuries on society is currently being discussed within both the New Zealand political and medical communities, and is highlighted by the following reports:

- Musculo-skeletal conditions in New Zealand - the crippling burden (Bossley \& Miles, 2009), which supports the Bone and Joint Decade initiative 20002010 (Bone and Joint Decade, 2012), was initiated by the World Health Organisation (WHO), and supported by the United Nations (UN). This document supports initiatives that are designed to manage the ageing population's issues with osteoporosis and joint movement, and trauma from bone injuries

- The ageing of New Zealand - an epidemic with major impact on musculoskeletal disease (New Zealand Orthopaedic Association, 2003), which outlines the issues surrounding the aging populations prevalence of osteoporotic fractures in the older adult and the need to have a strategic plan for the management of the increasing numbers of fracture events

- Fracture of neck of femur services in New Zealand hospitals 1999/2000 (Ministry of Health, 2002) highlights services across all District Health Boards (DHBs) in NZ as they relate to older adult hip fracture patients, which particularly focuses on time from admission to operation times, mortality rates and access to services. The results are compared against relevant best practice.

- Guideline for specialist health services for older people (Ministry of Health, 2004), which outlines supporting quality of care within the DHBs, by focusing on training staff, appropriate environments, ongoing quality improvement, and developing quality indicators.

- Targeting more elective operations - improved access to elective surgery (Ministry of Health, 2011), which emphasizes the need to manage all patients 
more effectively in order to achieve more elective surgery, by investigating ways of managing non-elective (emergency) patients better.

The report Fracture of neck of femur services in NZ hospitals 1999/2000 (Ministry of Health, 2002) has several questions for the health community that are thought relevant to this study. These questions are as follows:

- Given the observed regional variation in mortality for patients who suffer a [hip fracture], what can be done to improve the outcome?

- Are these patients given low priority for their surgery in our hospitals? Is this appropriate? Can it be changed?

- How can we encourage hospitals to ensure that the majority of hip fracture patients who are medically stable undergo surgery [by the following day] of fracture? (Ministry of Health, 2002, p. 27)

These questions are further qualified by the fact that currently only $27 \%$ of hip fracture patients are operated on within 24 hours of admission and $46 \%$ within the following 24 hour period (Ministry of Health, 2002).

The average life expectancy of a 65 year old person in NZ is approximately 85 years for women and 82 years for men, which is closely reflected in health statistics in the UK, US and Australia (Department of Health and Human Services, 2011). If you survive to 85 years, however, further life expectancy is predicted to at least 90 years of age (Statistics New Zealand, 2007). Caring for the older adult hip fracture patient, therefore, occurs at a time when they are nearing the end of their life span thus providing quality of life for this cohort of patient is a most pressing issue. It must also be acknowledged that mobility and thought processes are reducing at this age, and that a hip fracture event will reduce this further. 
The documents that have been outlined above will guide this study to gather data that will enhance information available about this cohort of patients. Key stakeholders will be able to formally assess current practice in a large NZ tertiary hospital and relevancy to NZGG recommendations and other international guidelines and research.

The aim of this thesis is to obtain data that will inform best practice for preoperative fluid management (PFM) in the older adult patient admitted with hip fracture. The outcomes of postoperative renal function, new reported postoperative confusion, timing of discontinuation of postoperative intravenous fluids indicating resumption of normal diet, and length of stay, were investigated with a methodology that was quantitative in nature. These outcomes have been chosen as they can be shown to be clinically meaningful, and intuitively be influenced by PFM. 


\section{Chapter 3: Methodology}

The study design incorporates the gathering of data from recognisable and accessible sources that are grounded in observational patient records, and deposited into a statistical program that will assist in useful biostatistical analysis. Results will be described using descriptive statistics and various forms of analysis that will compare variables and groups of variables.

\section{Key Question}

Does PFM of the older adult patient admitted with a hip fracture have a relationship with immediate postoperative outcomes among consecutive patients admitted to Wellington Regional Hospital (WRH) with a hip fracture, where PFM and postoperative outcomes are defined below?

\section{Definition of preoperative fluid management}

i) Time (hr) after admission when either oral or IV fluids are commenced.

ii) Preoperative oral fluid rate $(\mathrm{ml} / \mathrm{hr})$.

iii) Time (hr) before surgery that the last preoperative fluids were taken by the patient.

\section{Definition of postoperative outcomes}

a) Postoperative renal function, defined as significant change in GFR and serum creatinine between admission and $48 \mathrm{hr}$ postoperative.

b) Diagnosis of new reported postoperative confusion within $48 \mathrm{hr}$ following surgery.

c) Timing of discontinuation of postoperative IV fluids (hr), indicative of resumption of normal diet.

d) Total length of stay (days) in hospital. 


\section{Ethical approval}

Data gathered for this study was normally available for viewing by medical and nursing staff, but also by other relevant allied health professionals associated with the care of individual patients. This study involved the researcher gathering data from patient medical records outside her normal work area of operating theatre. Ethical approval for expedited review of observational study was, therefore, sought from the Central Ethics Committee (Appendix F), with approval gained (Reference number: CEN/12/EXP/009) on 13 ${ }^{\text {th }}$ February, 2012 (Appendix $\mathrm{G}$ ) and CCDHB Research Advisory Group (Maori) (Appendix H) in order for the researcher to be privy to this information. Data gathered will be utilised for this study only and patient consent or contact was not required.

\section{Study design}

This project is a prospective observational study that involved gathering data from older adult inpatients who had suffered a fragility hip fracture. Suitable patients for this study were notified from the acute surgical theatre list, and participant medical records were scrutinised immediately after the patient's stay in hospital. This study was undertaken within CCDHB, at both WRH and Kenepuru Rehabilitation Unit (KRU).

Inclusion criteria were patients aged 65 years and over who were admitted with an accidental fracture of the neck of the femoral bone and required surgical fixation. Exclusion criteria included hip fracture patients who did not have surgery, but were treated conservatively; had a pathological fracture of the femur without an associated fall event; or were undergoing treatment for malignancy or requiring palliative care only. Consecutive patients who were admitted with accidental fracture of the upper femur and neck of femur were studied over an approximately six month period from $1^{\text {st }}$ March, 2012. Target participant numbers were 100 patients, which provided data from just over $50 \%$ of patients admitted to CCDHB annually in this cohort. The age range of this patient group, 
and therefore the expected comorbidities, and admission numbers does not change greatly by month over the entire year, thus gathering data as stated is seen as an appropriate cross section for this study. Previous admission numbers for CCDHB in this patient group for 2010 and 2011 are 188 and 194 respectively, and average cases by month were 15.7 for 2010 and 16.2 for 2011 ( $\min 9$ - max 26, mean range 10.5 - 21.5 over the same two year period). No pattern for admissions was discerned with regards time of year, however, the maximum for 2010 (26) and 2011 (27) were in the spring months of September and October respectively (Spice, 2012).

\section{Participant access}

Access to appropriate patient medical records that fitted the characteristics of the study cohort was available via several routes. In the first instance a list of all appropriately coded patients (Table 3 ) from the operating theatre data base (ORSOS), was emailed three times per week by operating theatre IT staff to the researcher. Secondly the orthopaedic ward coordinator was approached to identify any possible patients that may be going to theatre for fixation of a hip fracture to ensure eligible patients were not missed. Patients were given study identification (ID) number if they fitted the inclusion criteria, and were admitted on or after the $1^{\text {st }}$ March 2012. 
Table 3

ORSOS codes for hip fracture surgery by type

\begin{tabular}{|c|c|c|}
\hline $\begin{array}{l}\text { ORSOS } \\
\text { code }\end{array}$ & Explanation & Surgery type applicable \\
\hline 4751900 & $\begin{array}{l}\text { Internal fixation of fracture of } \\
\text { trochanteric or subcapital } \\
\text { femur }\end{array}$ & Compression hip screw only (CHS) \\
\hline 4751900 & (as above) & $\begin{array}{l}\text { Dynamic hip screw and plate (DHS + } \\
\text { plate) }\end{array}$ \\
\hline 4753100 & $\begin{array}{l}\text { Closed reduction of fracture of } \\
\text { femur with internal fixation }\end{array}$ & Intramedullary nail (IM nail) \\
\hline 4752200 & Hemiarthroplasty of femur & Hemiarthroplasty \\
\hline 4931800 & $\begin{array}{l}\text { Replacement of head and } \\
\text { neck of femur, and } \\
\text { acetabulum of the pelvis }\end{array}$ & Total hip joint replacement (THJR) \\
\hline 4792100 & $\begin{array}{l}\text { Insertion of internal fixation } \\
\text { device, not specified } \\
\text { elsewhere }\end{array}$ & Screws and cables \\
\hline
\end{tabular}

\section{Pilot Work}

Sampling of 10 suitable patients was undertaken several months before initiating this project. This involved collecting data that the researcher thought might be useful to answer the key question and inform the outcomes. The same process was also undertaken again over the two months immediately prior to the start of data collection.

Several attempts to establish a succinct participant data collection form was made before the original final version 8 was confirmed (Appendix I). This was due to the desire to capture data that not only provided data about PFM, but also 
collected meaningful data concerning the demographics and perioperative journey of this cohort of patients. This version was subsequently altered to version 11 (Appendix J) during data collection, due to circumstances beyond the researcher's control, concerning patient being fit for discharge and ascertaining actual fasting time. Reference to fit for discharge was not documented formally or discussed in the progress notes, other than reference to suitable for discharge/rehabilitation when walking alone/drinking well by either a physiotherapist or orthogeriatrician. Deletion of fasting time is explained in chapter 4 under section for Collection issues and limitations.

The following table shows data topics that were deleted from original versions of the data collection sheet and those that were added to complete the final version (Table 4). 
Table 4

Data variables adjusted during pilot work

\begin{tabular}{|l|l|}
\hline Deleted & Reason \\
Fit for discharge & No clear indication in notes \\
Preoperative pain medication start time & Not directly relevant \\
\cline { 1 - 2 } Inserted & $\begin{array}{l}\text { Protein only clear renal function } \\
\text { indicator }\end{array}$ \\
\cline { 2 - 2 } Albumin & Reason \\
Preoperative oxygen administration & Relevant to malnutrition risk \\
Delay to OT & Known factor reducing confusion rates \\
Delay to admission & May affect confusion rate and LOS \\
Preoperative traction & Prolonged preoperative time \\
Urine rate pre- and postoperative & Known factor affecting confusion rates \\
Blood administered pre- or & Clear indicator of hydration status \\
postoperative & Indication of low Hb level \\
Urinary catheter insertion and removal & Known factor affecting confusion rates \\
Discharge destination & Suitability for DHB rehab or other \\
LOS at KRU & Compare total LOS with other \\
Dietitian referral & rehabilitation centres \\
\hline & Relationship with low Albumin level \\
\hline
\end{tabular}

Other changes that were made to the data collection sheet were in the printed order of variables to reflect the order of data within the patient notes and on line medical information. This was for ease of data collection. Alterations were also made to the page layout increasing or decreasing area available for data note taking, to ensure that the data collection form did not go longer than two pages.

The final group of data variables that were collected as part of this study is shown in the Data Analysis Grid (Appendix K). This grid indicates the status of 
each variable as a demographic, exposure, or outcome variable. Demographic variables included preadmission cohort characteristics of this older adult population, features of the hip fracture event, factors associated with the surgery, and other in hospital event data. Exposure and outcome variables are explained under the definition of PFM and postoperative outcomes earlier in this chapter.

The Data analysis grid indicates what markers of each variable have been recorded, but further notes to clarify data collection are recorded below:

- Postoperative biochemistry indicators were obtained as $48 \mathrm{hr}$ postoperative results but $24 \mathrm{hr}$ postoperative results were used if these were not available.

- Comorbidities were indicated as neurological if either physical (e.g. epilepsy or history of TIAs) or psychological (e.g. depression or anxiety); cardiac if patients suffered such conditions as atrial fibrillation or angina; dementia if preexisting cognitive impairment was present; and pneumonia included upper respiratory infection.

- Delayed admission to hospital was identified if patient had a fall and was not able to immediately contact assistance. Belated diagnosis of hip fracture was noted as delayed admission and related to patients who had either had continued pain in the affected hip or were unable to weight bear after a previous fall. Some of these patients had a prior x-ray with an undiagnosed hip fracture.

- Laboratory report that registered "trace" of protein in urinalysis was recorded as zero, however this is indicative of protein content of up to 20 $\mathrm{mg} / \mathrm{cm}^{-3}$.

- Administration of oxygen was recorded when it was documented in the progress notes, regardless of whether it was also prescribed on the MedR.

- Pain relief medication administered was noted directly from the patient MedR, which was not commenced until the patient was formally admitted. This mostly excluded those medications administered in A+E. 
- Recording of use of peripheral nerve catheter (PNC) for pain relief was done if any of the following types were inserted - lumbar plexus block, iliac fossa block, and femoral nerve block.

- The ASA code related to the American Society of Anaestheologists rating system for designation of patient health prior to surgery (Table 5). The addition of $E$ designates the patient as presenting for emergency surgery as opposed to planned surgery

Table 5

American Society of Anaestheologists rating scale

\begin{tabular}{|l|l|}
\hline ASA rating & Physical status \\
\hline I & $\begin{array}{l}\text { Healthy patient with no organic or psychological disease process, } \\
\text { and no systemic upset due to the pathological process for which } \\
\text { the operation is being performed }\end{array}$ \\
\hline II & $\begin{array}{l}\text { Patient with a mild to moderate systemic disease process, } \\
\text { caused by the condition to be treated surgically or another } \\
\text { pathological process, that does not limit the patient's activities in } \\
\text { any way, e.g. stable diabetic. Patients aged greater than } 80 \\
\text { years are automatically placed in this class or higher. }\end{array}$ \\
\hline III & $\begin{array}{l}\text { Patient with severe systemic disease from any cause that } \\
\text { imposes a definite functional limitation on activity, e.g. chronic } \\
\text { obstructive respiratory disease }\end{array}$ \\
\hline IV & $\begin{array}{l}\text { Patient with a severe systemic disease that is a constant threat to } \\
\text { life, e.g. unstable angina }\end{array}$ \\
\hline V & $\begin{array}{l}\text { Moribund patient unlikely to survive 24 hours with or without } \\
\text { surgery }\end{array}$ \\
\hline
\end{tabular}

(Gwinnutt, 2008)

- $24 \mathrm{hr}$ postoperative fluid totals and urine output rates included intraoperative totals. 
- Fluid type designated "milk" included all milky products, such as Milo, ice cream and custard, as well as supplementary drinks such as Fortisip (product trade name).

- Indwelling urinary catheter (IDC) was synonymous with application of urodome for male patients.

- Orthogeriatric assessment was acknowledged if done by either a specialist consultant or rehabilitation liaison nurse.

- Length of stay was designated primarily as time from admission to discharge from WRH.

\section{Method}

It was important to gather all relevant data for each patient so that the established data set was completed accurately. All staff associated with each patient, therefore, was seen as imperative to this study and how effectively they completed patient documentation. To this end all nursing staff and other healthcare professionals were alerted to the research study requirements.

\section{Staff education sessions}

An information session for nursing staff was undertaken prior to commencement of data collection. This was to ensure that they understood the purpose of the study and would endeavor to ensure patient documentation, such as fluid balance charts, would be accurately completed. Posters outlining the study was then attached to the ward office noticeboards for all healthcare professionals to access with the researchers contact details attached (Appendix $L$ ).

\section{Data gathering}

Following the start of the study date each patient record was scrutinised to source data for this study with information coming from the following sources:

- Adult vital Signs Chart (AVSC)

- Fluid balance chart (FBC) 
- Medication record (MedR)

- Anaesthetic record (AnR)

- Patient admission to discharge plan (PADP)

- CCDHB e-portal

- A+E record

- Discharge summary

- Operation report

- Laboratory reports

- Progress notes (daily reports)

- ORSOS

When patient data was missed due to early patient discharge information was gathered by way of recall of notes from medical records at a later date. When the patient was transferred to the KRU prior to 48 hours postoperatively notes were scrutinised by the researcher at KRU. A sample (anonymous) patient data collection sheet can be found in Appendix M.

\section{Quantitative analysis}

All data information was collated into the statistical package PASW Statistics 18, and analysis of data included the following:

- Descriptive statistics - mean, standard deviation (SD), median, and interquartile range (IQR), and minimum and maximum values for continuous variables (such as age and baseline observations), and distribution and frequency for discrete variables (such as gender and ethnicity).

- Statistical inference - to detect mean difference in preoperative and postoperative results a paired t-test was used. Significance was measured as $p \leq 0.05$. 
- Simple linear regression - to detect a relationship between the exposure variables of PFM and all outcome variables. Significance was measured as $p \leq 0.05$.

- Independent t-test - to detect mean difference in exposure variables and new postoperative confusion. Significance was measured as $p \leq 0.05$.

- Test of equality - McNemar's chi square test was used to ascertain test of equality between exposure and outcome variable groups. Significance was measured as $p \leq 0.05$.

- Multiple regression - analysis of a variety of independent variable groups to ascertain further predictive factors of each outcome variable.

Significance was measured as $p \leq 0.05$.

Utilising the above procedures analysis considered relationships between the exposure variables of PFM and each outcome variable as paramount to this study

\section{Reliability}

Actual documentation data content obtained during this study was presumed to be accurate and considered as such by the researcher. Cross checking of data obtained from patient documents was performed by the researcher against patient progress notes (which were hand written by nurses, doctors, physiotherapist, dietitians, and other healthcare professionals) where possible, comparing findings with AVSC, FBC, MedR, A+E notes, ORSOS, AnR, PADP, and laboratory reports.

\section{Validity}

Data collection by the researcher for this study could be readily re-obtained by independent personnel who had some affiliated healthcare training, due to the requirements to interpret medical comments and observed recordings. 


\section{Chapter 4: Results}

The results of findings from this study of older adult hip fracture patients admitted to CCDHB from March to September 2012 are presented as descriptive statistics of the participant cohort demographics, which include medical and surgical confounders, as outlined in chapter 3 . This is followed by explanations of the analysis of the relationships between the exposure and outcome variables. The complete Study data summary can be found Appendix M.

\section{Participants}

100 consecutively recruited patients admitted with a hip fracture that fitted the inclusion criteria were included in this study commencing on $1^{\text {st }}$ March 2012, and concluding on $17^{\text {th }}$ September 2012. Patients who had sustained a previous hip fracture on the same side as the current hip fracture were included if they had a diagnosis of hip fracture due to a fall event.

Patients were discharged after surgery from the acute orthopaedic ward at WRH and cared for during the postoperative rehabilitation process at either KRU or other chosen residential care setting. There were two patients who died after surgery but prior to discharge from WRH and one patient who died after admission to KRU. The participant profile of admission and discharges is shown in Figure 4. 


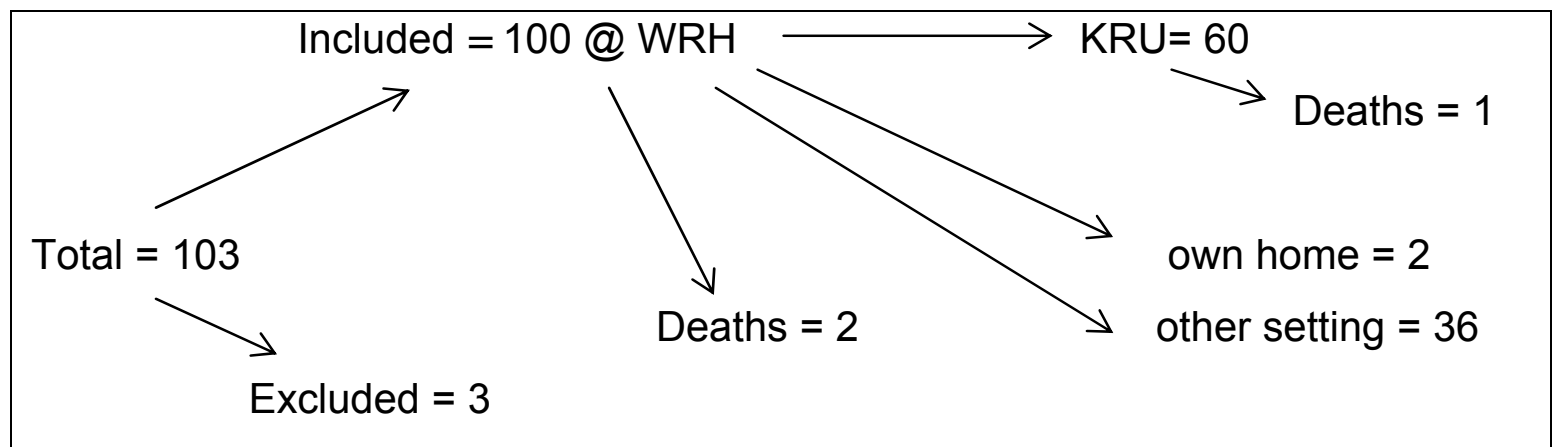

Figure 4: Participant profile

\section{Exclusions}

There were three patients admitted to the WRH during the study period and excluded from this study due to the following reasons:

- pathological hip fracture without an associated fall event (1)

- failure of a prosthetic device implanted for a previous hip fracture (1)

- nonunion of bone at previous hip fracture site requiring a replacement prosthetic implant (1)

All these patients, however, underwent surgery for repair of the hip fracture or hip fracture site.

\section{Demographics}

Information included within demographics was representative of data that described preadmission, admission and in hospital events.

\section{Age and gender}

The average age of hip fracture patients in this study was $85.2 \mathrm{yr}$ (SD 6.6, median $86, \min 68, \max 100$ ). $33 \%$ of this cohort was between 75 and $85 \mathrm{yr}$ of age, and $58 \%$ were $85 \mathrm{yr}$ of age and over. Patients were predominately female (70\%) who had an average age of $86.0 \mathrm{yr}$ (SD 6.1). Men had an average age of $83.5 \mathrm{yr}$ (SD 7.5). The following graph highlights the discrepancy in numbers between males and females in this study (Figure 5). 


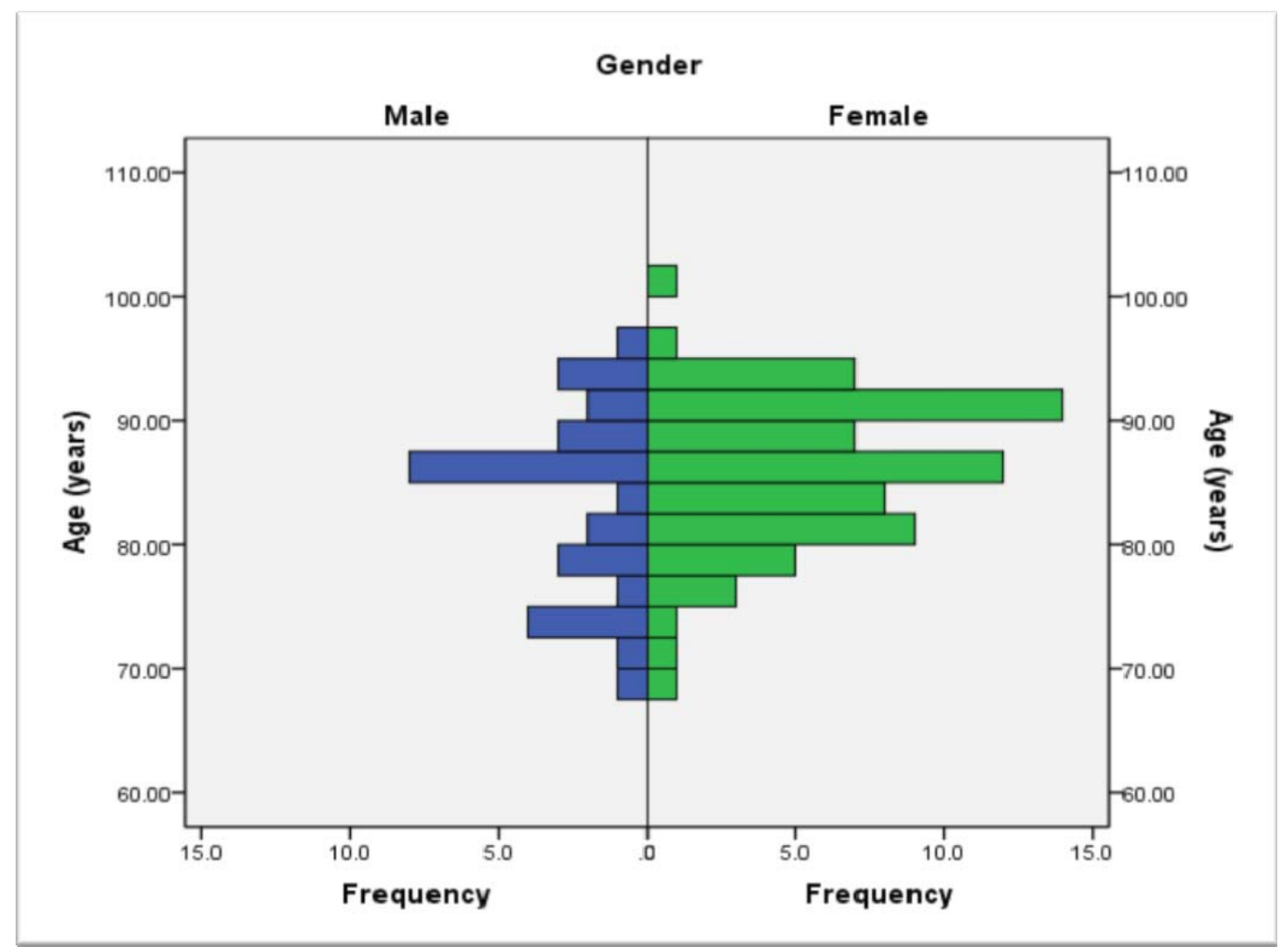

Figure 5: Males and females participants compared

\section{Ethnicity}

A total of 92 patients were of European extraction, the remainder were NZ Maori (1), Pacific Island decent (1), Asian (3) or of unknown ethnicity (3). Further designations of ethnicity were allowed for as per CCDHB admission protocol and itemised in the Data analysis grid (Appendix K) but not represented in these study results.

\section{Domesticity and discharge destination}

In this study 45 patients were admitted from their own home but just 2 were discharged directly to this destination. The remainder of patients was admitted from a residential care setting (21), hospital or hospice setting (19), or dementia designated unit (12). Two of the hospital based patients were also admitted from 
within $\mathrm{CCDHB}$, one from a medical ward at $\mathrm{WRH}$ and one from KRU. The later patient was recovering from a previous hip fracture. One further patient was designated as homeless.

Nearly two thirds of patients (61) in this study were discharged to KRU or other DHB rehabilitation setting. The remainder of patients was discharged to another residential care setting (11), hospital or hospice care (19) and dementia unit care (5). Just 2 patients were discharged directly to a home care situation. The significant difference between the numbers of patients discharged to a rehabilitation destination and those discharged to their original domestic environment are highlighted in the following graphs (Figures 6 and 7)

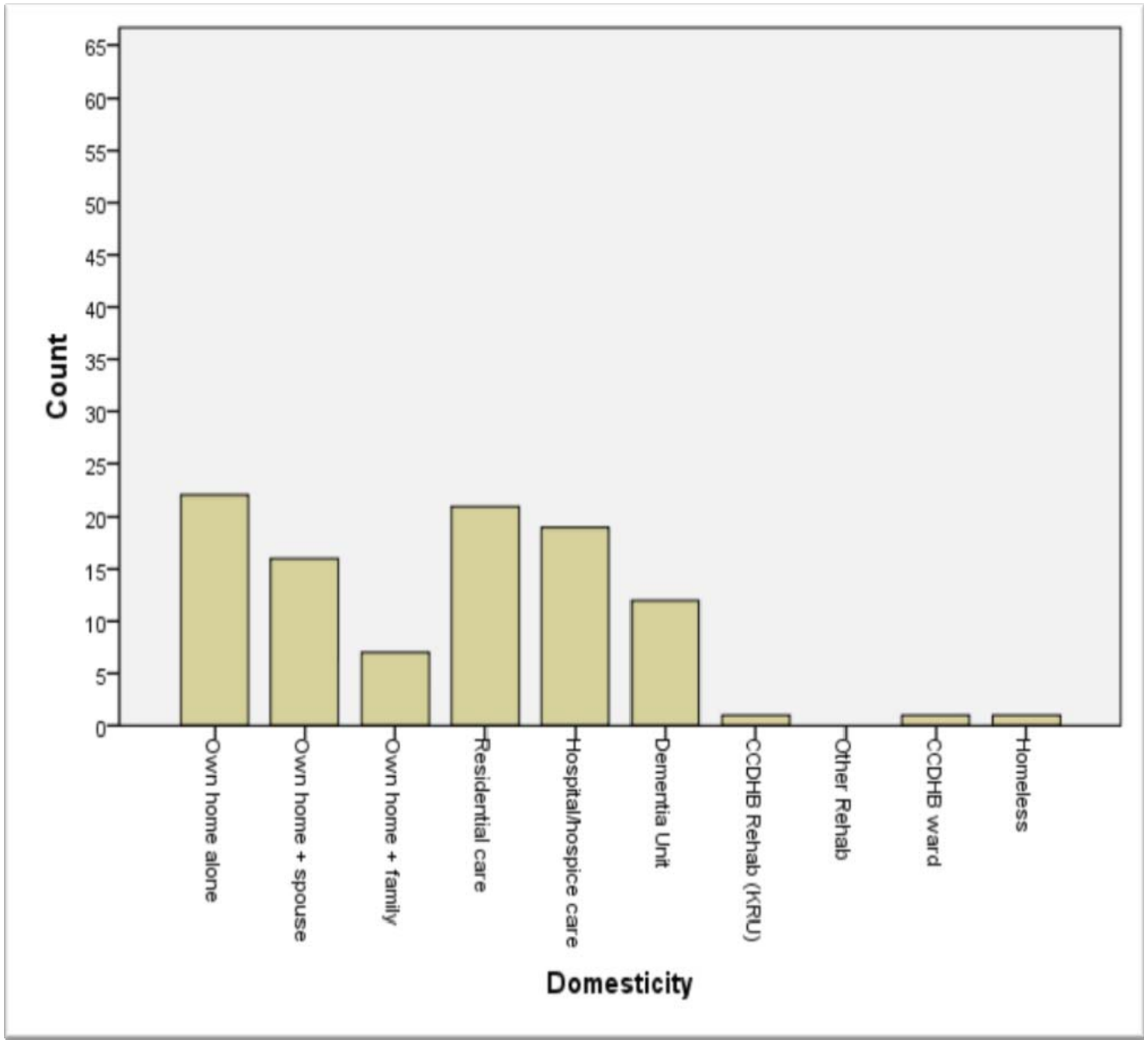


Figure 6: Domestic situation prior to admission

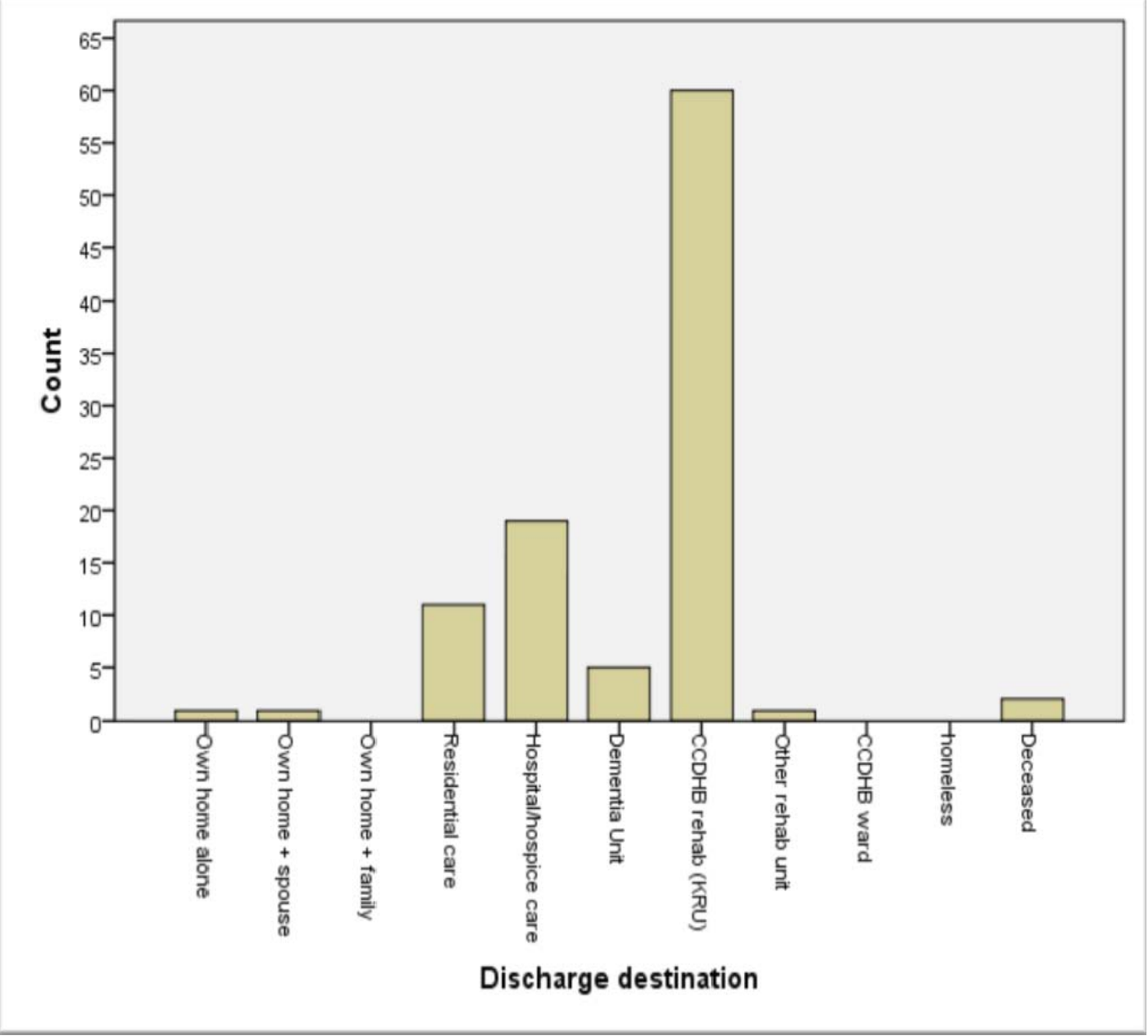

Figure 7: Participant discharge destination

\section{Comorbidities}

On admission patients had numerous comorbidities with cardiac pathology and dementia being the most prevalent. Cardiac issues were apparent in 58 patients within this study, and included angina, arrhythmias, CHF, previous cardiac valve replacement, bypass surgery, insertion of arterial stent, and IHD. This group did not include those patients who had hypertension (44) or hypercholesterolaemia (16). Dementia patients or those with significant cognitive impairment numbered 40 , with the majority of these patients being female (70\%). A further 28 patients 
had a physical or psychological neurological condition such as epilepsy, previous transient ischaemic attack (TIA) or depression. These figures did not include those patients who had suffered a previous stroke (17) or who had Parkinson's disease (6).

Other comorbidities apparent in this cohort of patients were pulmonary disease (22), vascular pathology (23), hypothyroidism (21), renal impairment (13), type 1 or $2 \mathrm{DM}(10)$, and gastrointestinal issues (19). Many patients also had acknowledged hearing (16) or vision loss (15) and 16 patients had suffered a previous hip fracture event. A current diagnosis of infection was found in a number of patients who had either pre-existing pneumonia (8), UTI (10) or other type of infection such as cellulitis or conjunctivitis (5). Anaemia was pre-existing in 7 patients and several more patients had a UTI diagnosed preoperatively (5),

A comorbidity score was calculated for each individual patient based on the number of the most common preadmission comorbidities found in this study. These included cardiac issues, hypertension, dementia, neurological, vascular, or pulmonary disease and hypothyroidism, renal impairment, DM (both type 1 and 2), anaemia, previous stroke and gastrointestinal issues. Percentages of patients with comorbidity scores are shown in Figure 8. 


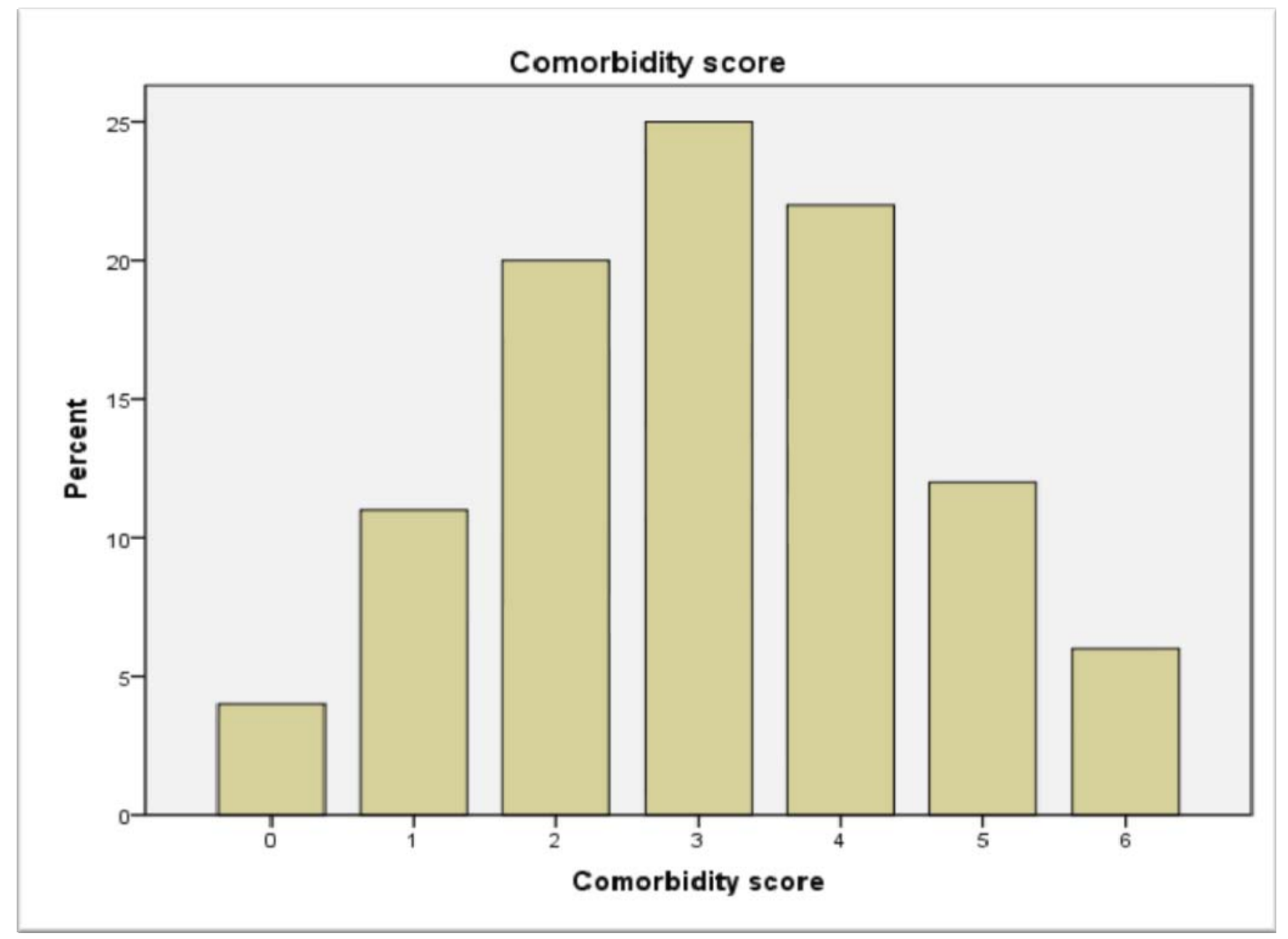

Figure 8: Patients comorbidity scores

\section{Medications}

The mean and median number of medications each patient had been prescribed prior to admission was 6.0 (SD 3.6, $\min =0, \max =16$ ). The most common drugs prescribed within this cohort of patients were for hypertension (49) and for anticoagulant therapy (40). Approximately 30 patients were each prescribed medication types for anxiety or depression, antipsychosis, diuresis, gastrointestinal issues, laxative, and analgesic properties. Antibiotics had been prescribed to 7 patients prior to admission, which accounted for some patients with acknowledged infections of UTI, pneumonia, or other infection on admission (5) and 2 patients who did not have an apparent infection. Bisphosphonates or supplementary calcium had been prescribed for the reduction in osteoporosis to 34 and 3 patients respectively prior to admission. 


\section{Baseline Observations}

The full summary of baseline observations for patients in this study with regards temperature $(T)$, pulse $(P)$, respirations $(R)$, systolic blood pressure (BPsys), oxygen saturation $\left(\mathrm{SpO}_{2}\right)$, and pain score appears in Appendix $\mathrm{N}$ - Study summary data.

Temperature, pulse, respirations, blood pressure and oxygen saturation Comparisons of mean differences of $\mathrm{T}, \mathrm{P}, \mathrm{R}, \mathrm{BPsys}$ and $\mathrm{SpO}_{2}$ between admission and $48 \mathrm{hr}$ postoperative data are outlined in below (Table 6).

Table 6

Mean admission and 48hr postoperative baseline observations

\begin{tabular}{|lccccc|}
\hline & $\boldsymbol{n}$ & Mean difference (SD) & $\boldsymbol{t}$ & $\boldsymbol{d f}$ & $\boldsymbol{p}$ \\
\hline Temperature (deg C) & 92 & $0.38(0.73)$ & 4.9 & 91 & 0.000 \\
Pulse (per min) & 94 & $4.0(13.5)$ & 2.8 & 93 & 0.006 \\
Respirations (per min) & 94 & $-1.4(4.1)$ & -3.3 & 93 & 0.001 \\
BP systolic (mm Hg) & 94 & $-28.8(35.1)$ & -7.9 & 93 & 0.000 \\
Oxygen saturation (\%) & 94 & $-0.8(5.6)$ & -1.7 & 93 & 0.091 \\
\hline
\end{tabular}

It is interesting to note that the mean increase in temperature between patients on admission compared with those at $48 \mathrm{hr}$ postoperative is only $0.38 \mathrm{deg} \mathrm{C}$, but in a paired $t$-test this is shown to be significantly different $(t=4.9, d f=91, p=0.00)$ in this sample. There is also a mean increase in pulse of 4 beats per min between patients admission recordings and those at $48 \mathrm{hr}$ postoperative and in a paired $t$-test this is shown to be significantly different in this cohort $(t=2.8, d f=93$, $p=0.006$ ). These results also show a slight decrease in respiratory rates of 1.4 per minute between admission and $48 \mathrm{hr}$ postoperative and in a paired t-test this is shown to be significantly different $(t=-3.3, d f=93, p=0.001)$. 
On admission BPsys was higher by $28.8 \mathrm{~mm} \mathrm{Hg}$ compared with recordings taken at $48 \mathrm{hr}$ postoperative, which in a paired t-test is significant in this cohort of patients $(t=-7.9, d f=93, p=0.00)$. The high mean admission BPsys $(151 \mathrm{~mm}$ $\mathrm{Hg}$ ) was well beyond the expected norm for a healthy adult of $120 \mathrm{~mm} \mathrm{Hg}$ and could have been indicative of patients being in pain and stressed. The $48 \mathrm{hr}$ postoperative mean BPsys (123 $\mathrm{mmHg}$ ) was very close to the expected norm and could have been more indicative of a medically controlled blood pressure. The patients were now predominantly pain free, appropriately hydrated and starting to resume normal ADLs.

Mean $\mathrm{SpO}_{2}$ dropped by nearly $1 \%$ between admission and $48 \mathrm{hr}$ postoperative, but in a paired t-test these results are not significant in this cohort of patients $(\mathrm{t}=$ - 1.7, $\mathrm{df}=93, \mathrm{p}=0.091)$. Normally expected $\mathrm{SpO}_{2}$ for a healthy adult would be 95-100\% saturation but does reduce with age. An older adult would still expect to have $\mathrm{SpO}_{2}>90 \%$ saturation but those with pulmonary issues may have a lower regular level.

\section{Pain score}

Preoperative patient pain score measurement was missing from nearly half of all patients (46) as indicated on the AVSC, yet comments about patients being in pain were recorded in patient progress notes. The following examples of comments taken from actual patient notes indicated pain for some patients where there was no score:

- Patient says she is sore.

- Regular pain medication required prior to intervention by physiotherapist. Of those 23 patients who did have a pain score documented on the AVSC 43\% appeared to have no pain (\#0) indicated, with 1 further patient scoring \#1, and 2 patients scoring \#10. Overall $57 \%$ of those patients scored $\leq \# 2,15 \%$ scored between $\# 3$ and \#4, and $22 \%$ scored between \#5 and \#6, Just $6 \%$ of patients scored $\geq \# 7$. Overall data for pain scoring was unable to be collected adequately as it was not undertaken using a formal assessment scale and many patients 
were designated as confused either preoperatively (48) or postoperatively (58) so may have been unreliable communicators of their pain.

\section{Laboratory results}

This section reports patient laboratory results of urinalysis, biochemistry $(\mathrm{Na}, \mathrm{K}$, and albumin), and haematology ( $\mathrm{Hb}$ and WCC). $\mathrm{Cr}$ and GFR are reported within the later section concerning Outcomes variables. A summary of all data results appear in Appendix N - Study summary data. Expected laboratory normals are itemised in Appendix $\mathrm{O}$.

\section{Urinalysis}

Just 23 of the 100 patients in this study had a urinalysis performed on admission. Mean urine specific gravity (USG) for these patients was within normally expected boundaries of $1.003-1.035 \mathrm{~g} / \mathrm{cm}^{-3}$. Slight proteinuria $(0.3 \mathrm{~g} / \mathrm{L})$ was detected in $5(22 \%)$ patients, significant proteinuria (1.0 g/L) was detected in 2 (9\%) patients, and the overall mean pH was acidic (neutral 7) at 6.09 (SD 0.9, $n=23$,).

\section{Biochemistry}

Comparisons of mean differences between admission and $48 \mathrm{hr}$ postoperative data for serum $\mathrm{Na}, \mathrm{K}$ and albumin are outlined in table 7 below.

\section{Table 7}

Mean admission and $48 \mathrm{hr}$ postoperative data for biochemistry

\begin{tabular}{|lccccc|}
\hline & $\boldsymbol{n}$ & Mean difference (SD) & $\boldsymbol{t}$ & $\boldsymbol{d f}$ & $\boldsymbol{p}$ \\
\hline $\mathrm{Na}(\mathrm{mmol} / \mathrm{L})$ & 98 & $-0.7(3.5)$ & -1.98 & 97 & 0.05 \\
$\mathrm{~K}(\mathrm{mmol} / \mathrm{L})$ & 98 & $-0.18(0.61)$ & -2.91 & 97 & 0.005 \\
Albumin $(\mathrm{g} / \mathrm{L})$ & 5 & $-8.8(5.5)$ & -3.55 & 4 & 0.02 \\
\hline
\end{tabular}


In this study there is a mean reduction in all biochemistry elements $(\mathrm{Na}, \mathrm{K}$ and albumin) from admission to $48 \mathrm{hr}$ postoperative. These results were all shown to be significant in a paired t-test. Results for $\mathrm{Na}$ and albumin at both admission and $48 \mathrm{hr}$ postoperative fell below recommended levels for these elements ( $\mathrm{Na} 139-$ $146 \mathrm{mmol} / \mathrm{L}$, albumin 34-46 $\mathrm{g} / \mathrm{L}$ ), and mean results for $\mathrm{K}$ fell within recommended levels $(3.5-4.9 \mathrm{mmol} / \mathrm{L})$. Only 21 patients had their albumin tested preoperatively and 29 postoperatively with just 5 patients being able to be included in the preoperative-postoperative comparison.

Haematology

Comparisons of mean differences between admission, $24 \mathrm{hr}$ and $48 \mathrm{hr}$ postoperative data for serum $\mathrm{Hb}$ and WCC are outlined in table 8 below.

Table 8

Admission, $24 \mathrm{hr}$ and $48 \mathrm{hr}$ postoperative data for $\mathrm{Hb}$ and WCC

\begin{tabular}{|lccccc|}
\hline & $\boldsymbol{n}$ & Mean difference (SD) & $\boldsymbol{t}$ & $\boldsymbol{d f}$ & $\boldsymbol{p}$ \\
\hline Hb & & & & & \\
Admission / 24 hr postop & 85 & $-21.6(16.6)$ & -12.0 & 84 & 0.00 \\
Admission / 48 hr postop & 58 & $-24.6(20.1)$ & -9.3 & 57 & 0.00 \\
$24 \mathrm{hr}$ postop / 48 hr postop & 45 & $-0.89(13.1)$ & -0.5 & 44 & 0.65 \\
WCC & & & & & \\
\hline Admission / 48 hr postop & 99 & $-0.06(9.18)$ & -0.07 & 98 & 0.943 \\
\hline
\end{tabular}

In this study using a paired t-test there is a significant reduction in $\mathrm{Hb}$ of $21.6 \mathrm{~g} / \mathrm{L}$ between admission and $24 \mathrm{hr}$ postoperative $(\mathrm{t}=-12.0, \mathrm{df}=84, \mathrm{p}=0.00)$ and $\mathrm{a}$ reduction of $24.6 \mathrm{~g} / \mathrm{L}$ between admission and $48 \mathrm{hr}$ postoperative $(\mathrm{t}=-9.3, \mathrm{df}=57$, $\mathrm{p}=0.00$ ). A further reduction in $\mathrm{Hb}$ of $0.89 \mathrm{~g} / \mathrm{L}$ between $24 \mathrm{hr}$ postoperative and $48 \mathrm{hr}$ postoperative is, however, not significant $(\mathrm{t}=-0.5, \mathrm{df}=44, \mathrm{p}=0.65)$. These results illustrate that on average patients had an $\mathrm{Hb}$ within normal parameters 
(115 - $160 \mathrm{~g} / \mathrm{L})$ on admission (124 g/L) but dropped well below within the $24 \mathrm{hr}$ postoperative period $(102.4 \mathrm{~g} / \mathrm{L})$ and further below during the $48 \mathrm{hr}$ postoperative period $(97.7 \mathrm{~g} / \mathrm{L})$.

In this study there is a reduction in WCC of $0.06 \times 10^{9} / \mathrm{L}$ between admission $\left(10.6 \times 10^{9} / \mathrm{L}\right)$ and $48 \mathrm{hr}$ postoperative $\left(10.51 \times 10^{9} / \mathrm{L}\right)$. These results are, however, not significant in a paired t-test $(t=-0.07, \mathrm{df}=98, \mathrm{p}=0.943)$ but are at the higher end of the expected range $\left(4.0-11.0 \times 10^{9} / \mathrm{L}\right)$.

\section{Operation details}

This section reports data that relate to the effects of surgery that may or may not have a compounding effect on the patient's wellbeing.

\section{Delay to admission}

The vast majority of patients $(n=100)$ were admitted to hospital within $24 \mathrm{hr}$ of the hip fracture event but 8 patients were delayed for a variety of reasons, including 2 patients that were discovered lying on the floor by either accident or by subsequent phone call. A further 2 patients were admitted for repair of their hip fracture 2-3 weeks following the hip fracture event due to a belated diagnosis, and 1 other patient who had a hip fracture diagnosed after a fall and damage to the other hip. A diagnosis of bilateral hip fracture was made in this latter case and both hips underwent surgical repair.

Delay to operation

A large number of patients (40) were reported to be delayed going to theatre for a variety of reasons. The reason for this delay was often not reported (29), but other patients were delayed due to an electrolyte imbalance needing correction (1), requirement for blood product replacement (2), reduction of clotting times of patients on anticoagulation therapy (2), cardiac issues (2), theatre management had more urgent cases (2), and 1 patient who was thought to require only 
palliative cares initially. Two of these aforementioned patients were postponed twice at approximately $9 \mathrm{pm}$. One further patient was not delayed to theatre and in fact prioritised due to difficulty in managing the patient's dementia behaviours in the ward, which involved aggression and spitting at the nurses, repeatedly pulling out of their IV line and urinary catheter, and disrobing.

\section{Preoperative oxygen}

Oxygen was administered preoperatively to 22 patients of which $6(27 \%)$ had a pre-existing pulmonary comorbidity. Most of the remainder of the preoxygenated patients (14) had cardiac issues, with a further 2 patients having neither cardiac nor pulmonary issues.

\section{Fracture type}

The most common type of hip fractures in this study were subcapital (51), followed by intertrochanteric (38), subtrochanteric (5), intracapsular (4), and transcervical (1). One patient also had a mid-shaft femoral fracture, below a previous hip fracture hemiarthroplasty repair. The patient who underwent bilateral hip fracture repair had a DHS and plate inserted on the left side and a right hemiarthroplasty. Sixteen patients overall who underwent hip fracture repair had had a previous hip fracture.

\section{Preoperative traction}

Patients who had traction applied prior to surgery for comfort and pain relief numbered just 14. Those patients who had traction had a range of fracture types and subsequent surgery with no apparent pattern.

\section{Operation type}

Most patients had either a hemiarthroplasty (48) or insertion of a DHS and plate (40) for repair of their hip fracture. Other patients had either insertion of a CHS only (1), IM nail (6) or THJR (4). The patient who had the mid-shaft femoral fracture had screws and cables applied for fracture fixation. 


\section{$A+E$ to operation}

The mean wait time from admission to operation was $35.3 \mathrm{hr}$ (SD 19.9, min 5.0, $\max 119.75, \mathrm{n}=100)$. Just under a third of patients (31) had their operation within $24 \mathrm{hr}$ post admission, with 85 patients being operated on within $48 \mathrm{hr}$. The following graph indicates $A+E$ to operation time in $6 \mathrm{hr}$ intervals (Figure 9) and showing a larger frequency of operations around the $24 \mathrm{hr}$ and the $48 \mathrm{hr}$ marks.

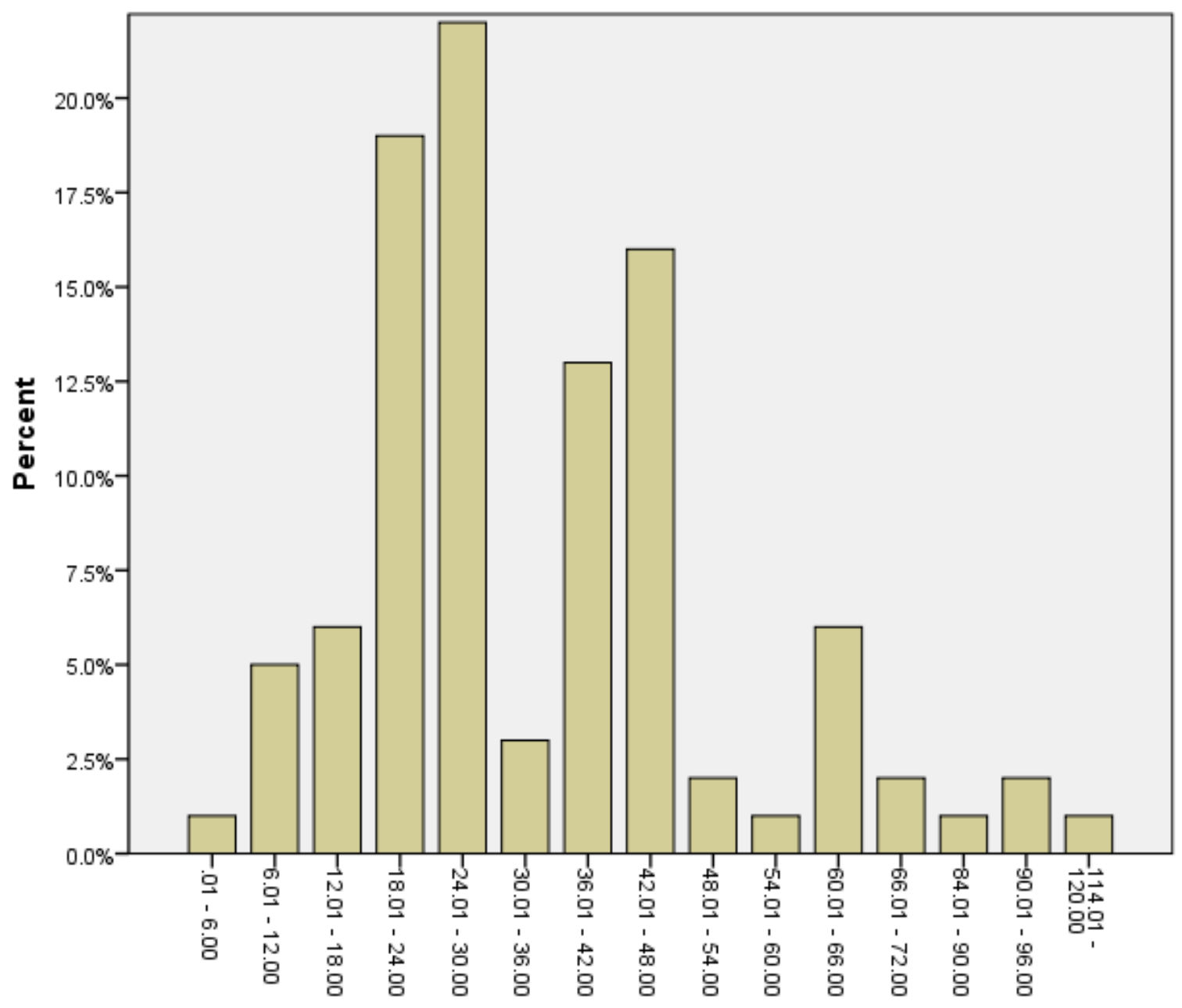

Figure 9: $\mathrm{A}+\mathrm{E}$ to operation time as per $6 \mathrm{hr}$ intervals

\section{Operation time}

The mean operation time was 73.3 min (SD 33.2) with a minimum operation time of $23 \mathrm{~min}$ and a maximum time of $184 \mathrm{~min}$ (approximately $3 \mathrm{hr}$ ) for unilateral hip 
fracture repair. The patient who had a bilateral hip fracture repair was operated on for $259 \mathrm{~min}(4.5 \mathrm{hr})$.

ASA

Most patients in this cohort had an ASA rating of III (71) or IV (21) indicative of severe systemic disease which imposes a definite functional limitation on activity, or is a constant threat to life. There was the need for just 2 patients to be admitted to ICU postoperatively for cardiac irregularities, with both returning to the ward before discharge. Two patients died postoperatively at WRH and one patient at KRU.

Anaesthetic type

General anaesthetic appeared to be the mode of choice for the majority of patients in this study (78), with just 13 having a spinal anesthetic only and 9 patients having a spinal anaesthetic with sedation during the operation.

\section{Hospital events}

This section reports data that may further enhance or compound the patient's surgical journey and are relevant to enhanced postoperative outcomes.

\section{Pain Relief}

Pain relieving medications recorded during this study were only those that could be verified by the MedR. As this documentation was only used in the ward, pain relief medication given in $A+E$ was not taken into consideration.

Most patients in this study had regular oral Panadol both preoperatively (91) and postoperatively (90) for pain relief, alongside oral Morphine tablets or syrup (57 preoperative and 58 postoperative). Other pain relief medication that was given preoperatively was Codeine (23), Fentanyl (17), Tramadol (8), and Oxynorm (3). 
Postoperative pain relief medication offered included Tramadol (23), Codeine (15), Pethidine (2), and Oxynorm (4).

Pain relief medication Marcain was also given via a peripheral nerve catheter (PNC), inserted either adjacent to the femoral nerve, in the lumbar plexus or adjacent to the iliac fossa in the hip area of the affected leg. Just 6 patients had this form of pain relief administered preoperatively in the ward, despite 64 patients having a PNC inserted prior in A+E. Intraoperative insertion of a PNC was done for 13 patients, and 11 patients had further administration of Marcain for pain relief postoperatively. 21 patients did not have a PNC inserted at all.

\section{Orthogeriatric assessment}

Assessment by a geriatric consultant specialising in orthopaedics, or a designated registrar or rehabilitation nurse specialist, was undertaken on each patient in this study within an average of 1.7 days (SD $=1.09$, $\min 0.4$, max 4.1) of admission, but 4 patients did not have assessment done at all during their stay in the WRH. Approximately half of all patients (51) were assessed prior to their operation and 45 were assessed within $24 \mathrm{hr}$ of admission.

\section{Indwelling urinary catheter}

Patients had an IDC inserted primarily in A+E (49), with a further 16 patients having an IDC inserted preoperatively in the ward and 13 patients intraoperatively. A further 2 patients had an IDC inserted postoperatively, with 5 patients having a pre-existing IDC before admission. There were 15 patients did not have an IDC inserted at all. Of those patients who had a catheter inserted in hospital either preoperatively or intraoperatively 77 (88\%) had a catheter remaining insitu at $48 \mathrm{hr}$ postoperatively and $51(55 \%)$ at discharge. The mean postoperative timing of removal of the urinary catheter was 3.9 days (SD 1.8, min 0.8 , max 9.3), with a total mean time insitu of 5.1 days (SD 2.2, min 1.6, $\max 11.9)$. 


\section{Dietitian referral}

Just 5 patients were referred for dietitian assessment either during their hospital stay or at discharge.

\section{Fluids}

This section reports data that is relevant to fluid management of the hip fracture patient but does not directly relate to the designated relationships of this study.

\section{Urine}

Both mean preoperative $(39 \mathrm{ml} / \mathrm{hr})$ and postoperative hourly urine rates improved during the patients' stay in WRH (Table 9). Significant gains in urine rates were found in a paired t-test between preoperative and both $24 \mathrm{hr}(\mathrm{t}=2.45, \mathrm{df}=66, \mathrm{p}$ $=0.017)$ and $48 \mathrm{hr}(\mathrm{t}=3.14, \mathrm{df}=52, \mathrm{p}=0.003)$ postoperative rates respectively $(49 \mathrm{ml} / \mathrm{hr}$ and $52 \mathrm{ml} / \mathrm{hr})$. It was also interesting to note that $30(\mathrm{~N}=100)$ patients in the preoperative period, and 16 patients in the period up to $24 \mathrm{hr}$ postoperatively, produced $<25 \mathrm{ml} / \mathrm{hr}$ of urine, which is the preferable minimum for a $50 \mathrm{~kg}$ person (Gwinnutt, 2008).

\section{Table 9}

Mean preoperative, $24 \mathrm{hr}$ and $48 \mathrm{hr}$ postoperative hourly urine rate

\begin{tabular}{|lccccc|}
\hline & $\boldsymbol{n}$ & Mean difference (SD) & $\boldsymbol{t}$ & $\boldsymbol{d f}$ & $\boldsymbol{p}$ \\
\hline preop / 24 hr postop & 67 & $9.39(31.32)$ & 2.45 & 66 & 0.017 \\
preop / 48 hr postop & 53 & $15.28(35.38)$ & 3.14 & 52 & 0.003 \\
24 hr postop / 48 hr postop & 68 & $2.83(31.19)$ & 0.75 & 67 & 0.457
\end{tabular}

Fluid types

Preoperative fluids were drunk by just $61(65 \%, n=94)$ patients during this study. Water was the predominant fluid offered to these patients with all of these patients having water preoperatively and $96(99 \%, n=97)$ of total patients having water postoperatively. This data takes into consideration missing FBCs of some 
patients (6 postoperative, 3 postoperative) and it is interesting to note that at least $33(35 \%)$ patients had no water at all preoperatively. Tea was the next most common beverage consumed by $23(25 \%)$ patients preoperatively and 61 $(63 \%)$ patients postoperatively, with coffee drunk by approximately $10 \%$ of patients both preoperatively and postoperatively. Both these products have diuretic properties so may affect hydration status. Milk products, considered a food substitute, were consumed by just $6(6 \%)$ patients preoperatively and 31 (32\%) patients postoperatively. The milk supplement Fortisip was predominantly drunk by those patients (3) who had it provided for by family members, with just 1 patient having it provided by hospital staff.

\section{Exposure variables}

Exposure variables of preoperative fluid management (PFM), as discussed in chapter 3, was measured by i) time after admission when either IV or oral fluids were commenced, ii) the preoperative oral fluid rate, and iii) time before surgery that the last preoperative fluids were taken by the patient.

\section{Start preoperative fluids}

Those patients who had preoperative oral fluids (60) had a mean start time of $11.4 \mathrm{hr}$ (SD 7.3, min 2.0, max 36.5) after admission, with just 30 patients having had any oral fluids within $6 \mathrm{hr}$ of admission. IV fluids were started slightly earlier than oral fluids on average at $9.6 \mathrm{hr}$ (SD 7.6, min 0.3, max 39.0) after admission, and just 38 patients had IV fluids commenced within $6 \mathrm{hr}$ of admission. Overall, however, either oral or IV fluids were commenced for half (50) of all patients within $6 \mathrm{hr}$ of admission, but there was a considerable range of start times extending up to $36.5 \mathrm{hr}$ for oral fluids and $39 \mathrm{hr}$ for IV fluids after admission.

\section{Preoperative oral fluid rate}

Patients had a mean hourly oral fluid rate of $11.7 \mathrm{ml} / \mathrm{hr}$ (SD 8.5, min 0.8, max 39.7 ) in the preoperative period. If fluids are reasonably anticipated to be 
consumed only between 6am and $9 \mathrm{pm}$ then a true mean preoperative fluid rate would be $18.7 \mathrm{ml} / \mathrm{hr}$, which is considerably less than the allowed volume as per the CCDHB perioperative adult fasting policy of $30 \mathrm{ml} / \mathrm{hr}$, and considerably more than reported in this study.

\section{Last preoperative fluids}

Last preoperative oral fluids were taken by patients $(n=32)$ on average $11.6 \mathrm{hr}$ (SD 5.8, min 2.0, max 24.8) prior to going to theatre for surgery. It is interesting to note that if patients were brought to theatre before midday they should be fasted for no longer than $6 \mathrm{hr}$ and just $6(22 \%)$ patients were in this situation. If patients were brought to theatre before $6 \mathrm{pm}$ then they should have been fasted for no more than $12 \mathrm{hr}$, and just 18 (46\%) patients were in this situation.

\section{Outcome variables}

Outcome variables are explained by identifying - a) postoperative renal function defined as significant change in GFR and $\mathrm{Cr}$ from admission to $48 \mathrm{hr}$ postoperative, b) new reported postoperative confusion, c) timing of discontinuation of postoperative IV fluids and resumption of normal diet, and d) total length of stay (LOS) at WRH.

\section{Renal function}

\section{Creatinine}

Mean serum $\mathrm{Cr}$ levels of patients within this study were $8.43 \mu \mathrm{mol} / \mathrm{L}$ less when measured at $48 \mathrm{hr}$ postoperative $(96 \mu \mathrm{mol} / \mathrm{L})$ compared with those at admission (104 $\mu \mathrm{mol} / \mathrm{L})$. This also indicated that renal function was predominantly within the expected range (62 - $103 \mu \mathrm{mol} / \mathrm{L}$ ) throughout their hospital stay, but at the upper end of normal. A paired t-test showed however, that there was a significant decrease in $\mathrm{Cr}$ in this patient group $(\mathrm{t}=2.426, \mathrm{df}=97, \mathrm{p}=0.017)$. Both preoperative $\mathrm{Cr}$ and postoperative $\mathrm{Cr}$ were not normally distributed however. 
GFR

Glomerular filtration rate is recorded up to 60 or $90 \mathrm{~mL} / \mathrm{min} / 1.73 \mathrm{~m}^{2}$, depending upon regional laboratory reporting schedules. At CCDHB GFR is reported as $\geq$ $60 \mathrm{~mL} / \mathrm{min} / 1.73 \mathrm{~m}^{2}$. To assist in the analysis of GFR, within this study, data has been grouped according to recognised levels of renal impairment as outlined in Appendix O: Laboratory normals.

On admission $47 \%(n=99)$ of patients had renal function of $<60 \mathrm{~mL} / \mathrm{min} / 1.73 \mathrm{~m}^{2}$ (level 3, $4+5$ ) which is below the level of mild renal impairment expected for an adult of advancing years. This improved at $48 \mathrm{hr}$ postoperative to $41 \%(\mathrm{n}=98)$ of patients. Results also show a reducing number of patients who had a GFR $<30$ $\mathrm{mL} / \mathrm{min} / 1.73 \mathrm{~m}^{2}$ (groups 4 and 5 ) from $12 \%$ to $7 \%$ between admission and $48 \mathrm{hr}$ postoperative, and a corresponding increase in number of patients with a GFR of $>30 \mathrm{~mL} / \mathrm{min} / 1.73 \mathrm{~m}^{2}$ (level 1, 2 and 3) at $48 \mathrm{hr}$ postoperative (93\%) compared with on admission (88\%).

\section{Confusion}

Preoperative confusion was reported in $47(n=99)$ patients, 57 patients within 24 $\mathrm{hr}$ postoperative, and 51 patients during the following $24 \mathrm{hr}$ period. The incidence of new postoperative confusion in those patients who did not have any reported preoperative confusion (52) occurred in 18(35\%) patients in this sample cohort.

\section{Postoperative IV discontinuation}

The mean time that IV fluids were continued postoperatively was $34.9 \mathrm{hr}$ (SD 29.3 , min 0.5 , max 156.25). Intravenous fluids were administered to 40 patients $(45 \%, \mathrm{n}=89)$ for up to $24 \mathrm{hr}$ postoperative, $28(31 \%)$ patients up to $48 \mathrm{hr}, 10$ (12\%) patients up to $72 \mathrm{hr}$, and $8(9 \%)$ patients up to $96 \mathrm{hr}$, with just 3 patients having administration of IV fluids $>96 \mathrm{hr}$ postoperatively (Figure 10). 
Discontinuation of postoperative IV fluids is indicative of return to normal eating and drinking.

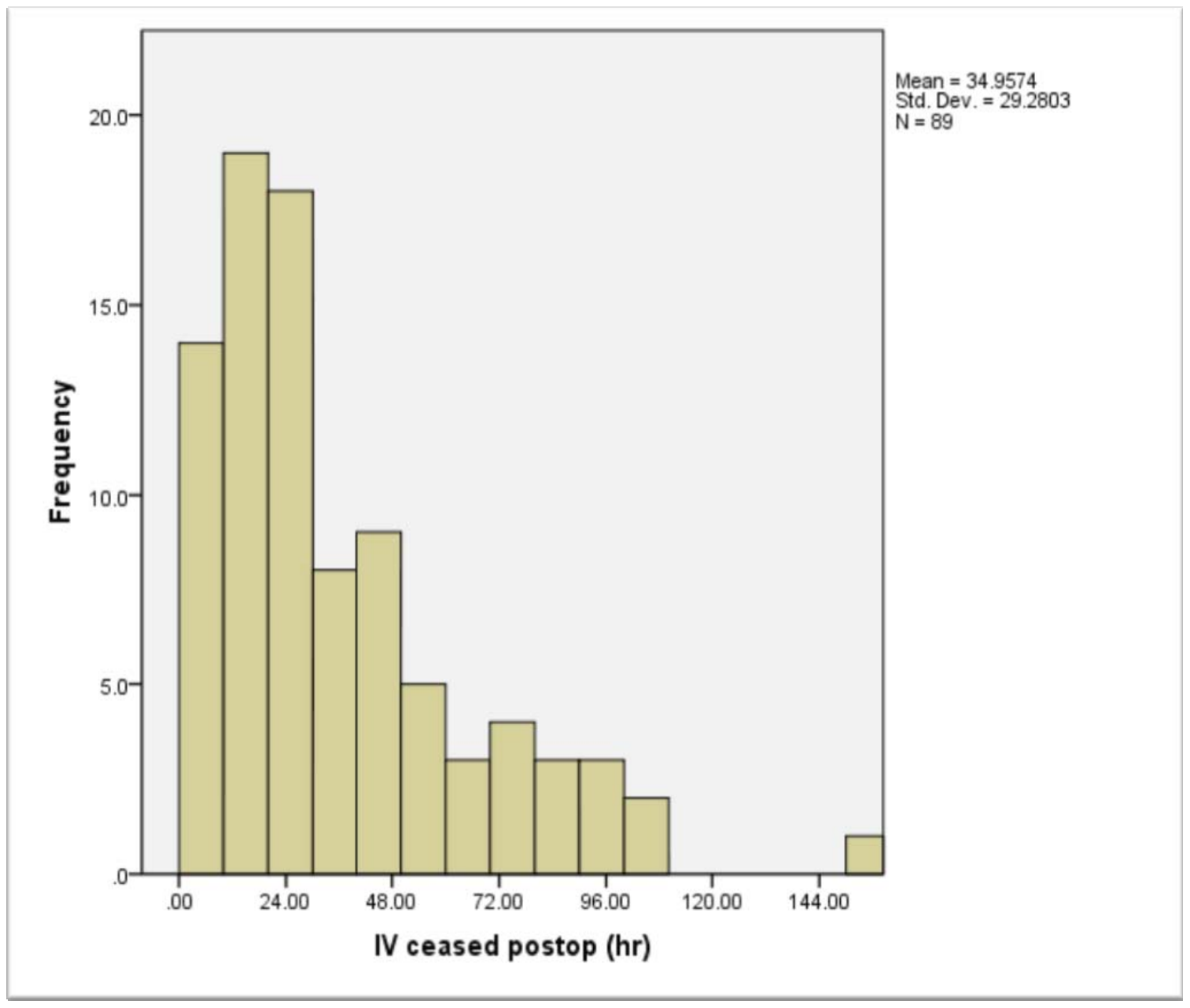

Figure 10: Postoperative IV discontinued

\section{Length of stay}

The mean LOS for surgical hip fracture patients $(n=98)$ in the acute orthopaedic ward at WRH was 6.7 days (SD 3.3, min 2.7, max 20.9) with a postoperative stay of 5.1 days $(S D=3.1, \min 1.6, \max 19.8)$. Patients who were transferred to KRU (57) for ongoing postoperative rehabilitation stayed a further mean time of 21.4 days (SD 12.9, $\min 3.0, \max 71.1$ ), and had a total CCDHB mean stay of 26.9 days (SD 14.1, $\min 8.0, \max 88.7$ ). 


\section{Relationships}

Relationships are explained by analysis of all PFM exposure variables with all outcome variables.. Simple linear regression and independent t-tests of analysis of mean was utilised to ascertain what type of relationship existed between the independent variables of PFM and outcome variables, with the probability of committing a Type 1 error (false positive) as the level of significance of 95\% ( $p \leq$ $0.05)$. GFR, however, was excluded from these forms of analysis as results were not reported precisely for those patients with a GFR $\geq 60 \mathrm{~mL} / \mathrm{min} / 1.73 \mathrm{~m}^{2}$. All exposure and outcome variables were further analysed in McNemar's test of equality to ascertain significant variable groups. Multiple logistic regression was then utilised to determine further significant variable groups that may predict each of the outcome variables.

\section{Linear regression}

PFM $v$ renal function

- Start preoperative fluids v $\mathrm{Cr} 48 \mathrm{hr}$ postoperative.

Analysis between how soon after admission preoperative fluids start and $48 \mathrm{hr}$ postoperative $\mathrm{Cr}$ shows that $\mathrm{Cr}$ increased by $0.29 \mu \mathrm{mol} / \mathrm{L}$ for every extra hour after admission that fluids start but that this result is not significant in this cohort $(\mathrm{t}$ $=0.331, p=0.742$ ). A scatter plot exhibits this lack of linear relationship, and the $R^{2}$ value shows that just $0.1 \%$ of the variability in the $48 \mathrm{hr}$ postoperative $\mathrm{Cr}$ is explained by the preoperative fluid start time. A Shapiro-Wilk test of normality shows that Cr levels are not normally distributed in this study $(p=0.000)$

- Preoperative oral fluid rate $v 48 \mathrm{hr}$ postoperative creatinine. Analysis of the preoperative oral fluid rate with the $48 \mathrm{hr}$ postoperative $\mathrm{Cr}$ showed that for every increase in $1 \mathrm{ml} / \mathrm{hr}$ of oral fluid consumed preoperatively the $48 \mathrm{hr}$ postoperative $\mathrm{Cr}$ increased by $0.735 \mu \mathrm{mol} / \mathrm{L}$ but this result is not significant in this group of patients $(t=0.799, p=0.428)$. The $R^{2}$ value shows that $1 \%$ of the variability in $48 \mathrm{hr}$ postoperative $\mathrm{Cr}$ is explained by the 
preoperative oral fluid rate and that the associated scatter plot shows no linear relationship.

- Last preoperative oral fluids v $48 \mathrm{hr}$ postoperative creatinine Analysis of last preoperative oral fluids and $48 \mathrm{hr}$ postoperative $\mathrm{Cr}$ shows that for every extra hour prior to surgery that last preoperative fluids were consumed serum $\mathrm{Cr}$ levels reduced by $0.227 \mu \mathrm{mol} / \mathrm{L}$ but this result is not significant in this cohort $(t=-0.1, p=0.921)$. A scatter plot also exhibits this lack of linear relationship and the $R^{2}$ value shows that that zero variability in the $48 \mathrm{hr}$ postoperative $\mathrm{Cr}$ is explained by the timing of the last preoperative oral fluids.

PFM v postoperative IV fluids discontinued

- Start preoperative fluids $v$ postoperative IV fluids discontinued Analysis showed that for every additional hour after admission that preoperative fluids were started the postoperative timing of discontinuation of IV fluids was reduced by $0.056 \mathrm{hr}$. This was significant in this cohort $(p=0.031)$, but not meaningful. A scatter plot exhibits this lack of linear relationship and the $R^{2}$ value shows that just $0.06 \%$ of the variability in the time postoperative IV fluids were discontinued is explained by the preoperative fluid start time.

- Preoperative oral fluid rate $v$ postoperative IV fluids discontinued Analysis showed that for every $\mathrm{ml} / \mathrm{hr}$ that preoperative oral fluids increased the postoperative timing of discontinuation of IV fluids increased by $0.045 \mathrm{hr}$ but is not significant in this cohort $(p=0.272)$. A scatter plot exhibits this lack of linear relationship and the $R^{2}$ value shows that just $0.02 \%$ of the variability in the time postoperative IV fluids were discontinued is explained by the preoperative fluid start time.

- Last preoperative fluids $\vee$ postoperative IV fluids discontinued 
Analysis showed that for every additional hour prior to surgery that the last preoperative fluids were consumed the postoperative timing of discontinuation of IV fluids increases by $0.001 \mathrm{hr}$ but is not significant in this cohort $(p=0.986)$. A scatter plot exhibits this lack of linear relationship and the $R^{2}$ value shows that just $0.00 \%$ of the variability in the time postoperative IV fluids were discontinued is explained by the preoperative fluid start time.

\section{PFM v LOS (WRH)}

- Start fluids post admission v WRH postoperative LOS Analysis between how soon after admission preoperative fluids start and total length of stay (LOS) shows that LOS decreases by 0.02 days for every extra hour after admission that fluids start but that this result is not significant in this cohort ( $p=0.646)$. A scatter plot exhibits this lack of linear relationship and the $R^{2}$ value shows that just $0.2 \%$ of the variability in the LOS is explained by the preoperative fluid start time. LOS is not normally distributed.

- Preoperative fluid rate $v$ WRH postoperative LOS Analysis between preoperative fluid rate and LOS shows that LOS increases by 0.052 days for every extra hour after admission that fluids start but that this result is not significant in this cohort $(p=0.314)$. A scatter plot exhibits this lack of linear relationship and the $R^{2}$ value shows that just $2 \%$ of the variability in the LOS is explained by the preoperative fluid rate.

- Last fluids preoperative $v$ WRH postoperative LOS Analysis between when the last preoperative fluids were consumed and LOS shows that LOS increases by 0.156 days for every extra hour after admission that fluids start but that this result is not significant in this cohort $(p=0.153)$. A scatter plot exhibits this lack of lineal relationship and the $R^{2}$ value shows that just $7 \%$ of the variability in the LOS is explained by when the last preoperative fluids were consumed. 


\section{Independent t-test}

PFM $v$ new postoperative confusion

The mean difference between those patients who did not get new postoperative confusion after analysis by independent t-test, and those who did, is itemised below (Table 10).

Table 10

PFM $v$ new postoperative confusion

\begin{tabular}{|l|l|l|l|l|l|l|l|l|}
\hline $\begin{array}{l}\text { New reported } \\
\text { postoperative } \\
\text { confusion }\end{array}$ & \multicolumn{2}{|l|}{ Yos } & \multicolumn{3}{l|}{ Mean difference } \\
\hline PFM & $n(\%)^{*}$ & $\begin{array}{l}\text { Mean } \\
(S D)\end{array}$ & $n(\%)^{*}$ & $\begin{array}{l}\text { Mean } \\
(S D)\end{array}$ & Mean & $t$ & $d f$ & $p$ \\
\hline $\begin{array}{l}\text { i) Start } \\
\text { preoperative } \\
\text { fluids (hr post } \\
\text { admit) }\end{array}$ & $\begin{array}{l}73 \\
(80)\end{array}$ & $\begin{array}{l}8.01 \\
(6.64)\end{array}$ & $18(20)$ & $\begin{array}{l}9.32 \\
(8.15)\end{array}$ & 1.31 & 0.718 & 89 & 0.475 \\
\hline $\begin{array}{l}\text { ii) Preoperative } \\
\text { fluid rate } \\
\text { (ml/hr) }\end{array}$ & 45 & $\begin{array}{l}10.68 \\
(78)\end{array}$ & $13(22)$ & $\begin{array}{l}15.32 \\
(8.82)\end{array}$ & 4.64 & 1.77 & 56 & 0.082 \\
\hline $\begin{array}{l}\text { iii) Last } \\
\text { preoperative } \\
\text { fluids (hr } \\
\text { preop) }\end{array}$ & $\begin{array}{l}(81) \\
\text { (1) }\end{array}$ & $\begin{array}{l}11.45 \\
(5.82)\end{array}$ & $6(19)$ & 12.5 & 1.05 & 0.396 & 30 & 0.695 \\
\hline
\end{tabular}

*\% of $n$ relates to those patients where data was available

- Start preoperative fluids $v$ new postoperative confusion

Patients who had no new postoperative confusion started either oral or IV fluids on average earlier after admission ( $8.0 \mathrm{hr}, \mathrm{SD} 6.64)$ than those patients who did 
get new postoperative confusion $(9.3 \mathrm{hr}, \mathrm{SD} 8.15)$ by $1.3 \mathrm{hr}$. This result, however, is not significant in this cohort $(p=0.475)$.

- Preoperative fluid rate $v$ postoperative confusion

In this cohort of patients it appears that those patients who did get new postoperative confusion had a higher preoperative oral fluid rate of $4.6 \mathrm{ml} / \mathrm{hr}$ than those patients who did not get new postoperative confusion but this result is not significant in this cohort $(p=0.082)$.

- Last fluids preoperative $v$ postoperative confusion

In this cohort of patients overall it appears that those patients who did not get new postoperative confusion had fluids closer to the time that they went to theatre by $1.1 \mathrm{hr}$ than those who did get new postoperative confusion. This result, however, is not significant in this cohort $(p=0.695)$.

Simple linear regression and independent t-test found that there were no significant or meaningful relationships between each PFM exposure variables and each outcome variable. Exposure variables were then grouped according to recognised parameters to examine relationships further.

\section{Tests of equality}

Exposure variables of PFM were grouped according to appropriate parameters and cross tabulated with grouped outcome variables with Pearson chi square test of equality using the binomial distribution.

The following exposure outcome cut-offs were set:

- Preoperative fluids start time was designated as $\leq$ or $>6 \mathrm{hr}$ after admission (maximum preferable time in $\mathrm{A}+\mathrm{E}$ ).

- Preoperative oral fluid rate was designated as $\leq$ or $>$ than $18 \mathrm{ml} / \mathrm{hr}$ (achievable hourly clear fluid rate over $24 \mathrm{hr}$ ). 
- Last preoperative oral fluids were designated as $\leq$ or $>$ than $6 \mathrm{hr}$ preoperatively (any fluids allowed up to this time).

The following outcome variables were grouped:

- Postoperative $\mathrm{Cr}>103 \mu \mathrm{mol} / \mathrm{L}$ (upper boundary of the expected norm).

- GFR $\geq 60 \mathrm{~mL} / \mathrm{min} / 1.73 \mathrm{~m}^{2}$ (boundary between level 2 and 3 renal function).

- New postoperative confusion or not.

- Postoperative IV discontinuation $>48 \mathrm{hr}$ postoperative (being half the median postoperative LOS and an indication of achievement of normal diet).

- LOS greater than 6 days (median total length of stay at WRH).

Chi square analysis with Pearson test of homogeneity between each exposure and each outcome variable grouped showed the following significant result:

- Preoperative oral fluid rate was significantly associated with new postoperative confusion $\left(X^{2}=4.435, \mathrm{df}=1, \mathrm{p}=0.035\right)$ with $\mathrm{OR}=0.252$ $(\mathrm{Cl} 0.067,0.955)$. This shows that the odds of having new postoperative confusion if your preoperative oral fluid rate is $\leq 18 \mathrm{ml} / \mathrm{hr}$ are $25 \%$ the odds of having new postop confusion if your rate is $>18 \mathrm{ml} / \mathrm{hr}$.

This result is not clinically intuitive with the role of fluids because $7 / 44(15.9 \%)$ of those with preoperative fluids $\leq 18 \mathrm{ml} / \mathrm{hr}$ did not have new postop confusion, and $6 / 14(42.8 \%)$ of those with preoperative fluids $>18 \mathrm{ml} / \mathrm{hr}$ did have new postop confusion. Your odds of having postoperative confusion, therefore, appear higher if you have a higher rate of preoperative fluids. 


\section{Multiple logistic regression}

Finally, multiple logistic regression techniques were used to further explore the relationship between the exposure and outcome variables that gave a significant result. Covariates added to the analysis were age, sex and comorbidity score. The analysis grouping is outlined below (Table 11):

\section{Table 11}

Predictors of new postoperative confusion

\begin{tabular}{|lcc|}
\hline New postoperative confusion & predictor coefficient & $p$ \\
\hline Preoperative oral fluid rate & -0.244 & 0.056 \\
Age & -0.013 & 0.140 \\
Gender (male) & 0.213 & 0.112 \\
Comorbidity score & 0.019 & 0.616 \\
\hline
\end{tabular}

Results of multiple logistic regression found that there were no further significantly independent predictors of new postoperative confusion.

\section{Summary analysis of variables relationships}

Analysis of variables showed no significant results were found in simple linear regression analysis of relationships between exposure variables of PFM and each outcome variable, however, grouping of variables and further analysis with tests of equality provided one surprising significant association between groupings of preoperative oral fluid rate and new postoperative confusion. Multiple logistic regression analyses suggested that there was no further predictor of new postoperative confusion after adjustment for preoperative oral fluid rate.

\section{Post hoc power analysis}

A post hoc power analysis to understand the risk in this study of committing a Type II error (false negative) can be done by estimating the size of sample 
required to establish significant results for testing differences between means of two groups with regards one outcome variable. With power equal to 0.80 there is a $20 \%$ chance of committing a Type II error.

By comparing whether preoperative fluids start $\leq 6 \mathrm{hr}$ after admission (group 1) or $>6 \mathrm{hr}$ after admission (group 2) with regards postoperative serum $\mathrm{Cr}$ the following calculation would be done to establish the estimated sample size (ES):

$$
\mathrm{ES}=\frac{\underline{\underline{\mu}} \underline{1}_{1}-\underline{\mu}_{2} \underline{\underline{I}} \underline{\text { Common SD }}}{\text { Common }}
$$

$$
\begin{array}{ll}
\mu_{1}=100.83 & \mathrm{SD}_{1}=63.6 \\
\mu_{2}=93.18 & \mathrm{SD}_{2}=50.34
\end{array}
$$

$E S=\underline{|100.83-93.18|}=0.13$

56.97

The ES is between 0.10 and 0.15 so to achieve a level of power of 0.80 would require a total of between 697 and 1568 participants divided between two equal groups.

\section{Collection issues and limitations}

Data collection for this study was an immensely frustrating exercise and was the main contributing factor to limitations. The key issues were around overall documentation, or lack of it, in the patient medical file, especially illegibility of inpatient progress notes, and incomplete $F B C s$ and reporting of confusion or pain scores on the AVSC.

\section{Patient medical file}

The patient medical file is bound together after discharge in a certain order which in general provided for ease of inspection of notes. In some circumstances, 
however, it was frustrating to find that the $F B C$ was not in date order or upside down, foldaway sections of the AVSC was secured within the binding of the file, and selected items were completely missing, for example, MedR or AnR.

Daily patient progress notes, held within the patient medical file, are hand written by nurses after each shift, as are notes by doctors and other health professionals who care for the patient. All these personnel have different handwriting, often use a myriad of abbreviations, and may not discuss their comments with other health care providers. Subsequently notes are illegible, they are often not read by other providers and consequently care to the patient may not be provided. One example of this was when a doctor instructed that a urinary catheter be removed from a patient and had to rewrite the instruction the following day because the request had not been followed up.

\section{Fluids and FBC}

There was a considerable amount of missing information from patients' $F B C s$ in regards pre- and postoperative oral fluids and it was often difficult to follow daily totals on the different $F B C$ forms and summary sheets (Appendix $\mathrm{P}$ and $\mathrm{Q}$ and $\mathrm{R}$ ) that were used for the hip fracture patients, with a large number of blank columns and daily pages missing. These gaps on the $F B C$ could have either been understood as "data not documented" or "no data to record", and 28 patients appeared to have nil oral fluids during the preoperative period. The researcher was also not able to utilise notes about fluids that were consumed in $\mathrm{A}+\mathrm{E}$, or noted on $A+E$ progress notes, as there was no $F B C$ commenced at that time.

The initial focus on percentage of NBM time was thought useful, with the expectation that patients would normally consume food and fluids between 6am and $9 \mathrm{pm}$, and the fasting policy recommending patients could have clear fluids until 6am. This type of recording was abandoned, after data collection had commenced, as it was found that FBCs did not itemise NBM time routinely and 
that medical staff was requesting that patients be NBM from either midnight or 2am, and occasionally 6am. The data collection recording sheet was consequently amended to include last preoperative fluids consumed to capture how well the fasting policy was being adhered to but 29 patient records were unable to be retrieved at this stage.

Prescription of IV fluids on the patient Medication Record (MedR) often did not relate to data on the $F B C$, as the MedR often did not have start or completion times recorded or total quantity given. Volumes of urine recorded on the $F B C$ were also found to be inaccurate when there was an indication that the patient was "UTT" (up to the toilet) or "PUB" (passed urine in the bottle) with no documented volume. These are further issues concerning accurate fluid recording and have quite major limitations on this study.

\section{Confusion reporting}

The AVSC was utilised in this study to gather data for baseline observations and level of consciousness parameters, one of which was agitation/confusion. A tick in this section was evidence of confusion. Unfortunately this section was often not completed or did not correlate with patients' progress notes. As there was initially no formal confusion scale in use in the orthopaedic ward searching through progress notes for details of confusion, disorientation or delirium was time consuming. Sometimes there was no indication of whether the patient was confused or orientated. Comments indicating to the researcher that a patient was confused, disorientated or delirious are recorded in Appendix S - Confusion indicators, as are comments that qualify or refute the diagnosis. Approximately halfway through the study a confusion scale was imbedded in the new Patient admission to discharge plan (PADP). This scale was not in regular use, however, so researcher knowledge of confusion, delirium and associated conditions continued to be utilised to ascertain confusion. This is seen as a major limitation to accurately ascertaining new reported confusion. 


\section{Chapter 5 - Discussion}

This study has focused on the relationship between PFM and outcomes of change in renal function, new reported confusion, postoperative IV fluid requirement, and LOS in the older adult hip fracture patient. In general, there was limited evidence of a relationship between aspects of PFM and the designated outcomes of this study, but this may reflect limited power to study associations in a cohort of generally well managed patients. Known research concerning this cohort in regard to demographics, perioperative and rehabilitation care, confusion and dementia, and accepted guidelines for the care of this older adult group have influenced this study, and is paramount in discussion of the study outcomes. These factors can now be discussed in association with current data and statistical information about the cohort characteristics and perioperative events as reported in this study. Concluding discussion statements can also be made concerning review of methodology, further research recommendations, and recommendations for patient care of the older adult hip fracture patient.

\section{PFM and postoperative outcomes}

CCDHB adopts the Perioperative fasting of adult patients policy, NZGG guideline statements, and discussed international research, concerning allowable preoperative clear fluids and preoperative hydration requirements. Preoperative fluids of any type have been recommended as appropriate up to $6 \mathrm{hr}$ prior to surgery and clear fluids up to $2 \mathrm{hr}$ prior to surgery. A further $30 \mathrm{ml} / \mathrm{hr}$ is permissible if required for medication but in fact may be appropriate when medication is not required, as it has been shown that a residual of $25 \mathrm{ml}$ of fluid in the stomach prior to surgery has no postoperative risk for the majority of adults (Brady, et al., 2003). Due to the fact that clear fluid travels through the stomach relatively quickly taking $30 \mathrm{ml} / \mathrm{hr}$ of clear fluids up to the time of being called for surgery may also have no postoperative ill effects for the older adult hip fracture patient. This suggests that adoption of the guidelines within CCDHB is generally 
associated with good clinical outcomes, although a number of recommendations based on observations made throughout this study are made later in this chapter.

\section{Demographics}

The older adult hip fracture patient has been found in this study to be associated with increasing age, often suffers from anaemia and infection, and other comorbidities that may have been a precursor to the hip fracture event.

\section{Age and gender}

The mean population age of $85 \mathrm{yr}$ found in this study of older adult hip fracture patients was above known cohort characteristics in overseas studies by between 2 and 5 yr where Björkelund, Hommel, Thorngren, Lundberg, and Larsson (2011) reported a mean age of $82.5 \mathrm{yr}$, Foss, et al. (2007) reported $83 \mathrm{yr}$, and Miura, et al. (2009) reported $80 \mathrm{yr}$. In recent NZ studies the mean age was found to be 82 yr by Davison, et al. (2001), 83 yr by the Ministry of Health (2002), and in 2007 by Fergus, et al. (2011) the mean age was found to be $84 \mathrm{yr}$. In studies of hip fracture patients those who were $85 \mathrm{yr}$ and over have been reported as $44 \%$ by Bottle and Aylin (2006) and as $42 \%$ by Björkelund, et al. (2011) but have been shown in this current study to be $58 \%$.

The proportion of females was $70 \%$ in this study at WRH which appears to be a significant reduction from previous hip fracture studies undertaken in NZ. A report to the National Advisory Committee on Health and Disability recorded $80 \%$ women (Sainsbury \& Richards, 1997) and in 2007 a large NZ public hospital study recorded $77 \%$ females by Fergus, et al. (2011). This decreasing rate of women could be in evidence due to the encouragement of doctors to prescribe women oestrogen hormone or calcium replacements and bisphosphonates for the reduction of osteoporosis and subsequent fractures (Sainsbury \& Richards, 1997). The prescribed use of either bisphosphonates or a calcium supplement 
prior to admission, however, was in evidence in just a third of patients in this current study.

\section{Domestic situation}

This study found that more than half of all patients (55\%) came from a preadmission domestic situation of some variation of hospital or residential care environment. This is considerably more than the $39 \%$ reported by Fergus, et al. (2011). Older adult care environments, therefore, need to be aware of simple ways to lessen the likelihood of hip fractures. Recommendations include more vitamin D, or access to sunlight, and regular physical exercise (Sainsbury \& Richards, 1997) to halt the course of osteoporosis. Ethnicity proportions in this study were slightly lower than expected for European patients at $92 \%$, but not surprising considering findings in the UK by Nematy, et al. (2006) of $96 \%$ European and in the US by Miura, et al. (2009) of 97\% European patients. There were just two patients in this current study that had difficulty in understanding English, both originating from continental Europe, and just one of these patients required an interpreter. This could have been an issue with understanding medical and care explanations as well as during the consent process.

Apart from a very small number of patients (2) who were discharged from WRH orthopaedic acute ward directly home the remainder had continued rehabilitation in a public or private hospital setting. This is consistent with the recommendation by the NZGG to provide a suitable postoperative rehabilitation environment. A large number of patients $(61 \%)$ were transferred to $K R U$ postoperatively which is considerably less than reported by Fergus, et al. (2011) who found that $84 \%$ of patients were transferred to a specialist older adult rehabilitation setting within the public hospital system. This could be indicative of patients in the CCDHB area having access to other appropriate hospital or residential settings that were capable of providing the required care. The capacity to provide physiotherapy and further postoperative care in the discharge environment was documented in 
patient notes. This is pleasing to see and it was noted a number of times in patient notes the preference to return to the preadmission place of residence for continuing rehabilitation by either the patient or family members.

\section{Comorbidities and prefall issues}

Significant medical issues were prevalent in this study cohort with $92 \%$ of patients having cardiac, pulmonary, vascular or renal comorbidities, including high blood pressure or evidence of an existing pneumonia. Systemic comorbidities that were found to be most prevalent were of a cardiovascular and respiratory nature which are significant preoperative risk factors for anaesthetic (Gwinnutt, 2008) and are also known to be major predisposing factors for postoperative complications of pneumonia and cardiac failure (Roche, et al., 2005). Both of these later medical issues were found in $72 \%$ of patients in this study, but there was evidence of just $13 \%$ of patients appearing to have no major comorbidity, which is similar to findings by Fergus, et al. (2011).

A majority of study patients had a high ASA rating, which is an indication of their comorbidity status, and was evidenced in the fact that a total of $92 \%$ of patients were rated ASA III $(71 \%)$ or ASA IV (21\%), which is indicative of severe systemic disease. These results were higher than those recorded by Fergus, et al. (2011) at $82 \%$ (ASA III $=68 \%$, ASA IV $=16 \%$ ) in 2007 . Care of these patients was no doubt challenging but effective to obtain an in hospital death rate in this study as low as $2 \%$. Other similar studies have recorded in hospital death rates of $14 \%$ by Bottle and Aylin (2006), 3\% by Björkelund, et al. (2011), and 5\% by Fergus, et al. (2011).

On admission to hospital electrolyte, haematology and baseline recordings in this cohort were generally found to be suboptimal in a significant number of patients. This could have been indicative of an undiagnosed comorbidity or medical issue, such as infection, which may have prompted the fall and hip fracture event, but 
certainly be a possible precursor to postoperative or rehabilitation issues. Baseline observations of hypovolaemia and hypoxia, which could have been possibly indicative of patient stress and dehydration around the time of the hip fracture, improved over the hospitalisation period.

Dehydration and malnutrition has been found to be an issue in the older adult population. Electrolyte analysis in this study showed that there was lower than normal expected values for $52(52 \%)$ patients on admission for $\mathrm{Na}$ as an indicator of dehydration and $15(71 \%)$ patients for albumin as an indicator of malnutrition in the preoperative period and also in the postoperative period ( $\mathrm{Na}=$ 62 , albumin $=29,100 \%$ ). It was disappointing to note here that this comprised most of the patients preoperatively and all of the patients postoperatively that were tested for serum albumin status. Furthermore over half of the 19 (63\%) patients who were admitted with preexisting indications of gastrointestinal disturbance did not have their albumin status tested during their stay in $\mathrm{WRH}$. Both reduced gastrointestinal uptake and reduced albumin levels may be indicative of dehydration and malnutrition (Martini \& Welch, 2006) and are known to affect the rehabilitation of hip fracture patients (Rai \& Mulley, 2007). In a study of perioperative nutritional intake of 100 hip fracture patients, evenly divided between a control group receiving the normal hospital dietary regime and an intervention group receiving a specified nutritional diet, Gunnarsson, et al. (2009) found that postoperative outcomes were significantly improved in the intervention group. The risk of postoperative UTI was reduced by half, other type of infection was reduced by a third, pressure ulcers were reduced by half, and LOS was reduced from 9 to 7 days. It is interesting to note here that just one patient in this current study received a supplementary liquid food from hospital staff and just 5 patients were referred for dietitian assessment despite known $\mathrm{Na}$ and albumin levels, and comments in the progress notes such as "appears frail". Improved nutrition stimulates the body and mind and provides energy for increasing mobility which is required to hasten the rehabilitation process and lessen on going postoperative problems. It is of concern, however, that the provision of 
supplementary liquid food is not routine for hip fracture patients as recommended by the NZGG.

Levels of serum $\mathrm{Hb}$ on admission in this study indicated that 24 patients preoperatively and 85 patients postoperatively were anaemic $(\mathrm{Hb}$ less than the expected norm of $115 \mathrm{~g} / \mathrm{L}$ ) and that the mean level of $\mathrm{Hb}$ fell significantly after surgery. This is of particular concern as just 27 patients overall had replacement blood products during their stay in hospital, and the ability of this cohort of patients to recover from surgery is considerably less than the younger adult due to their comorbidity status. A Cochrane Collaboration review by Carson, et al. (2012) reported that patients who had $\mathrm{Hb}<80 \mathrm{~g} / \mathrm{L}$ were advised to have blood products. Data from this current study reports that just $30 \%$ of those patients who had blood products had $\mathrm{Hb}<80 \mathrm{~g} / \mathrm{L}$ and $27 \%$ of these patients had $\mathrm{Hb} 80-90$ $\mathrm{g} / \mathrm{L}$ at some point during hospitalisation. The Cochrane Collaboration recommendation is being followed at CCDHB to some degree and further precautions are obviously taken with patients who have $\mathrm{Hb}>80 \mathrm{~g} / \mathrm{L}$. Unfortunately there were a further 2 patients who had postoperative $\mathrm{Hb}<80 \mathrm{~g} / \mathrm{L}$ that did not get blood products at all. Prescribing additional oxygen is also of benefit to anaemic patients, as they have reduced oxygen carrying capacity in the blood, but just $5(17 \%)$ of the preadmission and preoperative anaemic patients (30) had additional preoperative oxygen, despite this being an overall recommendation by the NZGG.

Mean haematological levels of WCC were at the high end of normal on admission, which is indicative of a possible current infection, in $40(40 \%)$ patients in this study, however, just 19 patients overall were recorded as having either a UTI, pneumonia or other type of infection, and just 7 patients were prescribed antibiotics prior to admission. Results of this study are similar to that of Bennett, et al. (2004) where $38 \%$ of older adult admissions to an emergency department had an acute infection, which predominantly included UTI and pneumonia. Infection of any type has been previously reported to be a precursor to a fall and 
hip fracture event in the older adult patient. This is consistent with this current study where a high number of hip fractures may have been avoided by identification of an infection prior to the fall event.

\section{Perioperative issues}

Hospital related events that the older adult hip fracture patient must undergo may be intrusive, add further risk to their recovery, or actually improve their preadmission health status. This study gathered data on a range of these issues which can be discussed within best practice guidelines. Insertion of an IDC, appropriate use of pain medication, administration of preoperative oxygen, and management of events surrounding the operation for hip fracture fixation are all highly relevant to this study cohort.

\section{Indwelling catheter}

Insertion of an IDC is not recommended in guidelines for this cohort of patients (New Zealand Guidelines Group, 2003) yet in this current study 80 patients had one inserted at some point during their hospital stay. During data collection this avenue did provide more accurate urine output recordings, for assessment of hydration status, but the chance of a postoperative UTI has been reported at $16 \%$ (Fergus, et al., 2011), with a higher chance of infection the longer it is left insitu. Olsson, et al. (2007), however, reported that there is the same chance of suffering a UTI whether it is insitu for a total of 3 or 6 days. The rate of UTI may therefore be associated with other variables, such as oral fluid rate, urine rate, personal hygiene or systemic comorbidity. Postoperative urinary retention may also be an issue for many patients with an IDC, which was certainly apparent in this study as some patients were noted to have had a trial of urinary catheter removal (TROCR) with repeated IDC reinsertion. Other issues associated with having an IDC inserted are immobility and being less reliant on accessing the toilet. Patients who were under a more rigid intervention regime to promote postoperative mobility, with encouragement to have no IDC or have it removed 
within one day of surgery, have been shown to have half as many pressure ulcers develop as those who had standard care (Olsson, et al., 2007). Intervention patients also had a shorter LOS (3 days) to standard care patients, who had a similar LOS (5 days) as this current study ( 6 days). Insertion of IDC, predominantly during the preoperative period, appears to be standard practice in this current study and $90 \%$ of those patients still had an IDC insitu $48 \mathrm{hr}$ postoperatively. It was noted during data collection, however, that a few patients had an IDC longer than required due to miscommunication of postoperative medical orders requesting removal. Usage of an IDC in this study cohort appears, therefore, to need some discussion amongst healthcare professionals.

\section{Pain relief}

Medication to relieve pain effectively for the hip fracture patient has preoperative and postoperative advantages to manage bone and surgical pain, and to encourage postoperative mobilisation. Options to manage pain need to be adjusted carefully to avoid over medicating the older adult patient with excessive opioids which can cause confusion or lethargy. Equally, medication needs to be given regularly to ensure pain relief is adequate while waiting for surgery and to be prepared for postoperative physiotherapy.

In this current study a PNC with Marcain was utilised for pain relief in 79 patients overall, the majority $(64,81 \%)$ being inserted in $A+E$. Just a few patients, however, had additional Marcain added either preoperatively (2) or postoperatively (11). This form of pain relief generally gives good effect for up to $6 \mathrm{hr}$ and is utilised alongside regular Panadol as a very effective pain management regime. It is particularly useful with patients in this cohort who have a high chance of preexisting dementia or becoming confused during their hospital stay and may not be able to adequately explain or demonstrate their pain level. Other forms of pain relief were utilised but some patient progress note comments 
confirmed the inadequacy of pain relief by physiotherapists in the postoperative period:

- Unable to proceed with mobilisation treatment due to the patient being in pain.

- The patient was too drowsy to be cooperative.

Mobilisation, as discussed earlier, is a key requirement of rehabilitation in order to combat postoperative complications, such as pneumonia or pressure ulcers. Pain relief, therefore, is an important consideration in patient management and needs to be gauged appropriately, preferably using a recognised technique.

Measuring pain level with an appropriate scoring system is necessary for all age groups and especially for older adult patients who may have cognitive impairment. Preoperative pain scores were missing for nearly half of all patients in this current study and there were also progress notes comments indicating that the pain score was subjective only, such as:

- Discussion with patient who says pain is ok.

- Patient says he is sore.

Pain management is, therefore, a challenging issue which may compromise patient quality of life as well as decreasing effective preoperative and postoperative comfort and rehabilitation.

\section{Operation details}

Time from admission to surgery for the hip fracture patients in this study averaged $35 \mathrm{hr}$ (median $=29.25 \mathrm{hr}$ ), which was considerably longer than the recommended timeframe of $24 \mathrm{hr}$. Just 31 patients underwent surgery within the first $24 \mathrm{hr}$ after admission and just over half (56) had their operation within $36 \mathrm{hr}$. Nearly a third of the patients (29) had an indication of delay or postponement to surgery for no specific reason documented in their progress notes, and half of the patients (3) who had a preadmission delay had a further delay documented, but some of these were for medical reasons (2). The prioritisation system operating 
for acute surgical patients at CCDHB ranges from category \#1, which needs to be operated on within $2 \mathrm{hr}$, to category \#4, which needs to be operated on within $24 \mathrm{hr}$. Undergoing surgery within one day of admission, however, may be impractical in some situations where the patient is not medically stable, or there is a significant back log of acute patients and high priority cases. This recommendation, however, should ensure that patients in this cohort are assessed as a priority, preoperative treatment requirements are efficiently adhered to, and surgery for the majority of patients are within $24 \mathrm{hr}$. The majority of patients had a general anaesthetic (GA) for fixation of the hip fracture (78) with the remainder having a spinal anaesthetic with or without sedation with just 2 postoperative deaths ( $1 \mathrm{GA}, 1$ spinal). This is indicative of the capabilities of this older adult age group when medical intervention is appropriately applied and where length of operation and type of fracture are irrelevant to the immediate postoperative outcome.

\section{Confusion and dementia}

Confusion in this cohort may be exacerbated by being out of their usual residential environment, together with the stress of the hip fracture event, and a possible infection or undiagnosed medical issue. Dementia patients require careful consideration while in hospital as they are away from their normal environment and regular routines. Cognitive impairment or diagnosis of preexisting dementia occurred in a large number of patients (40) admitted into this study cohort, a further 17 patients were assessed as having preoperative confusion, and a further 10 patients reported as having new postoperative confusion. The latter group of patients did not have any type of preexisting cognitive impairment or reported preoperative confusion. It has been reported by Fergus, et al. (2011) that $43 \%$ of patients admitted with a hip fracture had cognitive impairment, which is similar to this current study also in a NZ tertiary hospital. Nearly half of the remaining patients in this current study, however, had reported confusion at some point during their hospital stay, making a total of 67 
patients having either dementia or confusion. Looking after this cohort of patients, therefore, requires knowledge of the subtle differences between the signs and symptoms of dementia and confusion.

There was no formal assessment scale for confusion which could be accessed when gathering data for this study. This is of real concern as care concerning this issue is recommended to be proactive with formal assessment strategies (New Zealand Guidelines Group, 2003). Supporting documentation in progress notes suggesting confusion may in fact have related to behaviour of a dementia patient (see Appendix R: Confusion indicators and Appendix C: Features of delirium and dementia compared). Caring for this cohort of older adult patients, therefore, requires staff to be vigilant to changes in behaviour, and knowledge of a variety of specialised assessment and care options in order to provide quality and effective treatment.

\section{Review of methodology}

The overall observational methodology engaged in this study was seen as appropriate considering that the data to be collected needed to reflect actual occurrences in patient treatment and care. This was achieved and the results are seen as realistic, as opposed to contrived. Further strengths of this study include the gathering of comprehensive data about hip fracture patients admitted to CCDHB over more than six month. This information could be utilised to research relationships of a variety of variables in the future.

One weakness of the methodology of this study was the reliance on other personnel to collect the data as previously discussed in Chapter 4 - Collection issues and limitations. A further weakness relates to the lack of ability to reveal causal relationships, such that results are open to faulty interpretation, and may be affected by a pre-existing difference in clinical status of an individual patient that provides a plausible alternative explanation. This type of reverse causality 
could explain the significant results that in reality appear counterintuitive in practice.

Considerations to improve the methodology for future research into this cohort of patients might involve a randomised controlled trial. This would be necessary to infer causality in the relationship between preoperative fluid management and postoperative outcomes by providing a control or "normal" cohort sample of similarly aged patients who are not undergoing surgery for hip fracture.

\section{Future research recommendations}

Data gained from this study suggests that to establish causality association between preoperative fluid management and postoperative outcomes a randomised intervention would be required.

\section{Patient care recommendations}

Ideal care and treatment options for patient care have been mentioned throughout this research report. These are further itemised below:

- Practice improvement to include directives from policies and recommendations for guidelines could be implemented using a recognised methodology to assist all healthcare practitioners.

- Assessment of fluid replacement incorporating a dehydration check list which includes researched parameters, compulsory admission urinalysis testing and an algorithm for treatment.

- Fluids offered hourly by healthcare assistants if nursing staff are unavailable

- Education for all healthcare professionals to appreciate the difference and be able to assess confusion and dementia signs and symptoms, alongside a regular formal assessment scale which is common in all areas of the hospital. 
- Progress notes to be computerised to enable all healthcare professionals to easily relate to previous patient care and treatment.

- Pain assessment to be assessed using an appropriate method which includes number scoring, face charts and behaviour indicators.

- Individual patient care plan (IPC) incorporating mobilisation, preoperative and postoperative fluid regimes, infrequent IDC, and a designated ward area for hip fracture patients.

- Admission to operation time planning that relates to the designated priority rating.

\section{Concluding statement}

Inconclusive results have been found in this study regarding preoperative fluid management and postoperative outcomes in older adult patients admitted with hip fracture, possibly due to limited sample numbers. However, a significant amount of data has been gained concerning the demographics and care of this cohort of patient in a NZ tertiary hospital. This could be utilised to manage these patients more effectively and to promote utilisation of recommended guidelines. 


\section{Appendix A}

Best practice evidence-based guideline summary: Acute management and immediate rehabilitation after hip fracture amongst people aged 65 years and over - summary guidelines (NZGG)

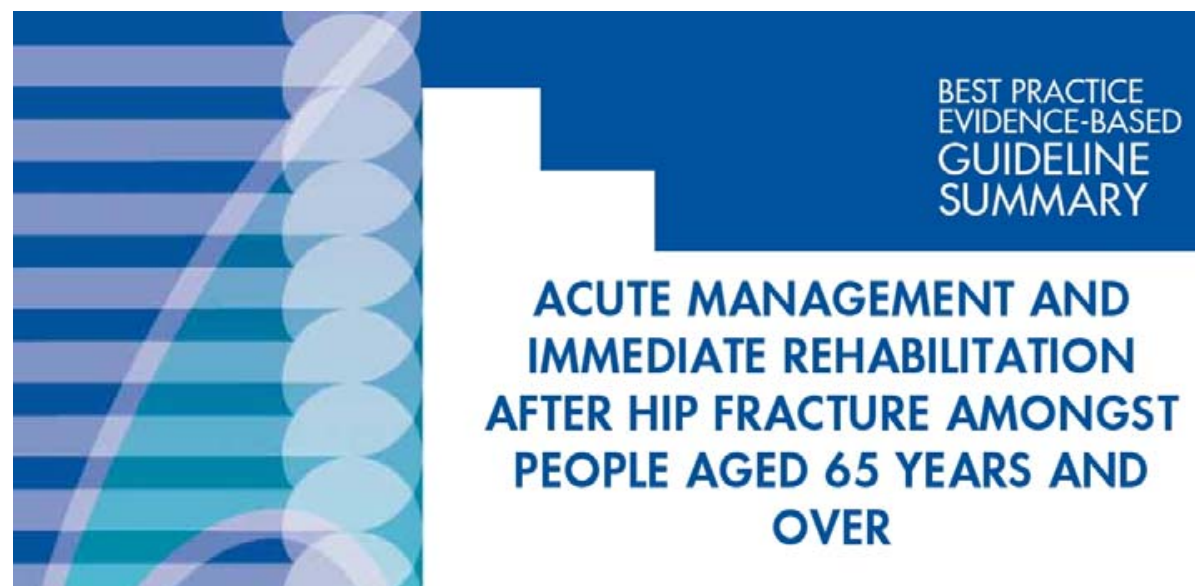

This guideline provides an evidence-based summary of the acute management and rehabilitation of older people after they have sustained a hip fracture.

The consequences of hip fractures in older people create a significant and increasing burden of illness in the community, and can precipitate a dramatic decline in physical function. Twenty percent of older people who sustain a hip fracture die within a year. Two years after the fracture, survivors are more than four times more likely to have limited mobility than people of similar age without a fracture, and more than twice as likely to be functionally dependent.

Evidence shows that the early treatment of older people with hip fracture is effective. The guideline team has thoroughly researched the evidence and makes recommendations in the following areas:

- pre-hospital care

- emergency department care

- ward care

- surgical management

- immediate rehabilitation.

A second guideline has been developed for Prevention of Hip Fracture Amongst People Aged 65 Years and Over and is available from the New Zealand Guideline Group's website (www.nzgg.org.nz).

Endorsed by

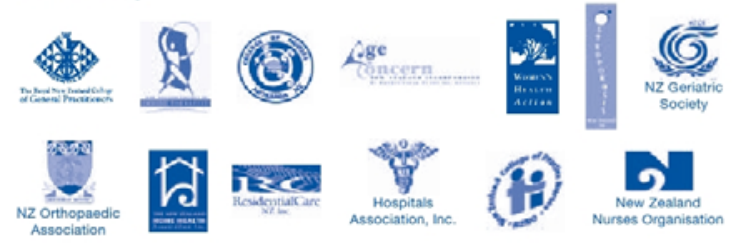

Supported by

Australasion Colloge for Emergency Medicine 


\section{RECOMMENDATIONS FOR HIP FRACTURE MANAGEMENT}

\section{PRE-HOSPITAL CARE}

\section{Pre-hospital Care}

- In isolated areas, fluid replacement and catheterisation prior to transport to hospital may be indicated.

\section{EMERGENCY DEPARTMENT CARE}

\section{Emergency Department Management}

- Hospitals treating hip fracture should have formal 'fast track' protocols for assessment and admission of people aged 65 years and over.

\section{Fluid Management}

- After hip fracture, there is a risk of dehydration because of inability to gain access to sufficient fluids. Careful fluid management is required, as there is also risk of fluid overload when fluid replacement is given intravenously.

\section{Pre-operative Traction}

- Routine use of temporary leg traction appears to be unnecessary.

\section{Pain Relief}

- Use of systematic pain assessment tools helps to avoid undertreatment or overtreatment of pain.

- As frail older people tolerate narcotics poorly, multiple modalities should be considered for analgesia.

- Narcotic use must be carefully titrated and supervised.

- Paracetamol should be preferred to aspirin as their effects are similar milligram for milligram, but paracetamol has fewer side effects.

- Ibuprofen is an NSAID effective in post-operative pain, and appears to have lower incidence of adverse effects than other NSAIDs.

- Propoxyphene-containing compounds are not recommended in people aged 65 years and over with hip fracture.

- The use of local analgesic nerve blocks reduces the need for parenteral or oral analgesia.

\section{Oxygen Therapy}

- Oxygen should be administered to maintain adequate tissue oxygenation, as indicated by oximetry and clinical status. 


\section{WARD CARE}

\section{Prophylaxis Against Venous Thromboembolism}

- Adequate fluid balance and early post-operative mobilisation lower the risk of post-operative venous thromboembolism (VTE).

- Administration of either aspirin or low molecular weight heparin is associated with reduced risk of VTE, but some increase in adverse bleeding events.

- Foot or calf pumps reduce the incidence of VTE, but have some adverse skin effects and compliance problems.

- There is insufficient evidence to confirm the effectiveness of thromboembolism stockings after hip fracture.

\section{Antibiotic Prophylaxis}

- Antibiotic prophylaxis is effective in reducing wound infection after hip fracture surgery.

\section{Preventing Pressure Sores}

- The use of high specification foam bed mattresses and pressure relieving mattresses on operating tables reduces the incidence of pressure sores.

\section{Nutritional Supplementation}

- Oral multinutrient feeds reduce unfavourable outcomes (death or post-operative complication) after hip fracture.

\section{Urinary Tract Management}

- Routine catheterisation after hip fracture is not recommended.

- When urinary retention occurs, intermittent catheterisation results in quicker restoration of normal voiding than indwelling catheterisation.

\section{Managing Dementia/ Delirium}

- Initial admission data should include a formal measure of cognitive function.

- Early involvement of a geriatric medical team in hip fracture care has been associated with a significant reduction in the incidence of post-operative delirium.

- Active re-orientation by provision of clock, calendar, radio, television and telephone does not appear to reduce post-operative cognitive deterioration.

- Continuity in nursing care may reduce post-operative cognitive deterioration.

\section{GUIDELINE DEVELOPMENT TEAM}

This guideline was developed by William Gillespie (Convenor), John Campbell, Melinda Gardner, Lesley Gillespie, Jan Jackson, Clare Robertson, Jean-Claude Theis and Raymond Jones. The consultation group included Marion Robinson, Heather Thomson and Jim Reid.

Published by New Zealand Guidelines Group Inc.

An electronic copy of the full guideline is available for download from www.nzgg.org.nz or a printed copy is available from info@nzgg.org.nz, phone 64-4-471 4180 or Box 10-665, Wellington, New Zealand. 


\section{SURGICAL MANAGEMENT}

\section{Delay Before Surgery}

- Early operation (within 24 hours) for people aged 65 years and over with hip fracture is associated with shorter hospital stay and decrease mortality/morbidity.

\section{Anaesthesia}

- Regional anaesthesia for hip fracture surgery is associated with a lower rate of deep venous thrombosis than general anaesthesia, but no significant differences in mortality or other measures of morbidity.

\section{Surgical Management of Undisplaced Intracapsular Fractures}

- Screws appear to provide better fixation and fracture healing than unthreaded pins.

\section{Surgical Management of Displaced Intracapsular Fractures}

- Arthroplasty is associated with a lower re-operation rate than internal fixation.

- In arthroplasty after hip fracture, the use of bone cement may be associated with less late pain in the limb.

- Unipolar hemi-arthroplasty appears as effective as bipolar hemi-arthroplasty, and is less expensive.

- There is insufficient evidence to identify whether the use of total hip replacement is superior to the use of hemi-arthroplasty in displaced fracture of the femoral neck.

\section{Surgical Management of Extracapsular Fractures}

- Fixation with a sliding hip screw gives superior results to fixed nail plate devices, or intramedullary devices.

\section{Surgical (Suction) Wound Drains}

- The usefulness of surgical suction wound drains after hip fracture surgery is unproven.

\section{Post-operative Mobilisation}

- People with hip fracture should be mobilised, weight bearing with support as tolerated, as soon as possible after surgery.

\section{IMMEDIATE REHABILITATION}

\section{Early Rehabilitation}

- Hospitals providing treatment for people aged 65 years and over with hip fracture should provide formal hip fracture programmes which include early multidisciplinary assessment by a geriatric team.

- Early Supported Discharge Programmes reduce mean hospital stay, and are associated with a higher rate of effective return to previous residential status. 


\section{Appendix B}

Perioperative fasting of adult patients (Policy PER-05, CCDHB)

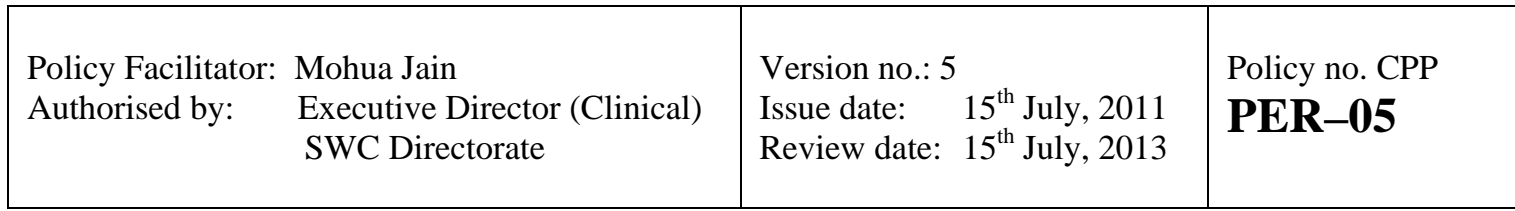

\section{Perioperative Fasting of Adult Patients}

\section{Related C\&C DHB documents}

Perioperative Management of Diabetes Protocol - Adults (CPP PER-11)

When you child needs Anaesthesia

\section{Introduction}

This policy relates to the pre-operative fasting of adult patients - elective day cases, elective day of surgery admissions, acute admissions and inpatients.

The aims are to maximise comfort, minimise risk and adverse outcome and to promote perioperative efficiency.

\section{Scope}

All C\&C DHB staff involved with perioperative care including:-

Medical

Nursing

Technical

Midwifery

\section{Indications}

This policy applies to all adult patient groups (elective and emergency) requiring anaesthesia or intravenous sedation administered by C\&C DHB anaesthetists.

\section{Exceptions}

Current exceptions may include (due to differing perioperative management):-

1. Cardiac surgery patients (earlier starting times)

2. Patients for elective cataract surgery (most likely under local anaesthesia) 
3. Patients with acute gastrointestinal disorders (who may be fasted for treatment anyway)

4. Patients requiring bowel preparation prior to procedures (different instructions for stopping food)

5. Other elective or urgent surgery where set starting times are difficult to define - advice will be given on a case by case basis.

\section{Procedure}

No changes should be made to the following instructions without consultation with the patient's anaesthetist (+/- surgeon, +/- physician).

\section{Fluids}

Intake of water up to 2 hours before induction of anaesthesia for elective or urgent surgery is safe in the majority of adults and has been shown to improve patient wellbeing.

There is increasing evidence that the volume or rate of drinking does not matter, however, a sensible current reference recommends one standard glass $(200 \mathrm{mls})$ per hour upto 2 hours before the procedure.

(Although other true clear fluids are also safe up to two 2 hours before, only water is recommended in this policy for the sake of safety and simplicity).

\section{Solids}

Solid foods, milk, tea/coffee with milk, sweets/lollies, chewing gum, enteric feeding are acceptable up to 6 hours before induction of anaesthesia.

(A minimum preoperative fasting time of 6 hours is recommended).

\section{Medications}

Regular oral medication should be continued pre-operatively unless there is advice to the contrary.

There will be special management of patients on anti-coagulants, anti-platelet agents, some antihypertensives or diabetic medication (see Perioperative Management of Diabetes Protocol - Adults)

Up to 30 mls water may be given orally to help patients take their medication if less than 2 hours before induction.

\section{If the patient is scheduled for a procedure on a morning list}

Stop all food from midnight on the day before surgery. Water should continue until 0600.

Drinking until later in the morning may be appropriate but must be authorised by the patient's anaesthetist. 


\section{If the patient is scheduled for a procedure on an afternoon list (which cannot possibly start before midday)}

- A light breakfast (eg. cereal/toast/fruit, tea/coffee/milk but not fried or fatty food) may be given on the morning of surgery, so long as it is finished by 0600 .

- Water should continue until 1000.

- Drinking until later in the morning may be appropriate but must be authorised by the patient's anaesthetist.

\section{If the patient is scheduled for a procedure on an all day list}

- Treat as for morning surgery, unless otherwise advised.

- There will be staggered times up to which food and water may be permitted, depending on when a patient is scheduled. Ideally this should be established as early as possible on the day of surgery, only after multidisciplinary discussion and providing no variation is likely. If in doubt or if no changes are advised, default to the morning instructions and check later.

\section{Risks and precautions}

\section{Higher risk groups for possible regurgitation}

- $\quad$ those in pain

- diabetic patients

- $\quad$ extremes of age

- pregnant women

- those who are obese

- trauma/emergency patients

- those with a history of acute or chronic gastrointestinal disease

Note that this is not an exhaustive list.

It is now recommended that these patients should follow the same preoperative fasting regime as healthy adults, unless advised by medical staff.

This

may help with gastric emptying.

(In the past, this group were recommended to be fasted for longer, because of their higher risk of delayed gastric emptying, but the evidence no longer supports this).

Note - Patients with acute bowel obstruction or ileus will often be nil by mouth (NBM) for 
therapeutic reasons, regardless of whether they are to have procedures or not (see point 3 under Exceptions)

However, it is still essential for the anaesthetic team to consider further pre- and intraoperative interventions for these higher risk patients, as appropriate to the overall clinical situation.

\section{Note}

As with all clinical policies, recommendations may not be appropriate for use in all circumstances. Decisions to adopt any particular recommendations must be made by practitioners in the light of:

- available resources

- local services, policies and protocols

- the patient's circumstances and wishes

- the clinical experience of the practitioner

- knowledge of more recent research findings

It is important for all health care professionals that, when implementing evidence-based guidance, they understand the local context in which they work and existing quality improvement structures.

\section{References}

- Perioperative Fasting in Adults and Children - Royal College of Nursing (RCN) Guideline, November 2005, with updates on www.rcn.org.uk - with endorsements by Royal College of Anaesthetists (RCA), Association of Anaesthetists of Great Britain \& Ireland (AAGBI) and other key organisations

- Recommendations for the Pre-Anaesthesia Consultation - PS7 Professional Document of Australian \& New Zealand College of Anaesthetists (ANZCA), December 2010

- Recommendations for the Perioperative Care of Patients Selected for Day Care Surgery PS15 Professional Document of ANZCA, December 2010

- Pre-Operative Assessment and Patient Preparation (The Role of the Anaesthetist) AAGBI Safety Guideline, January 2010

- Aspiration - Is There an Optimal Management Strategy? N H Cohen, Chapter 49, Evidence-Based Practice of Anesthesiology, edited by L A Fleisher, $2^{\text {nd }}$ edition, 2009 


\section{Appendix C}

Features of delirium and dementia (section from Dementia guidelines, Policy MAN-27, CCDHB)

TABLE 1 - Features of delirium and dementia (adapted from Pitt, 1998)

\begin{tabular}{|l|l|l|}
\hline Feature & Delirium & Dementia \\
\hline Onset & Acute & Insidious \\
\hline Course & Fluctuating & Consistent \\
\hline Duration & Hours to weeks & Months to years \\
\hline Lucid spells & Common & Early - occasional \\
\hline Sundowning & Always & Often \\
\hline Awareness & Reduced & Clear \\
\hline Alertness & Heightened or reduced & Usually normal \\
\hline Attention & Fitful, distractible & More sustained \\
\hline Sleep-wake cycle & Always disrupted & Occasionally disrupted \\
\hline Orientation & Variably impaired & $\begin{array}{l}\text { More consistently } \\
\text { impaired }\end{array}$ \\
\hline Memory & $\begin{array}{l}\text { Immediate and recent } \\
\text { impairment }\end{array}$ & $\begin{array}{l}\text { Recent and remote } \\
\text { impairment }\end{array}$ \\
\hline Speech & $\begin{array}{l}\text { Variably rambling and } \\
\text { incoherent }\end{array}$ & $\begin{array}{l}\text { Early - losing words } \\
\text { Late - incoherent }\end{array}$ \\
\hline Hallucinations & Common, visual & Occasional \\
\hline Delusions & Fleeting & $\begin{array}{l}\text { Early - more sustained } \\
\text { Late - absent }\end{array}$ \\
\hline Affect & Labile, intense & Often normal or apathetic \\
\hline Physical illness & Usual & Occasional \\
\hline Recovery & Common & Very rare \\
\hline & &
\end{tabular}




\section{Appendix D}

\section{Fractured Neck of Femur (\#NOF) - Fast track form (CCDHB)}

Capital \& Coast

District Health Board

OPOKO KI TE URU HAUORA

\section{Fractured Neck of Femur (\#NOF)}

Fast Track

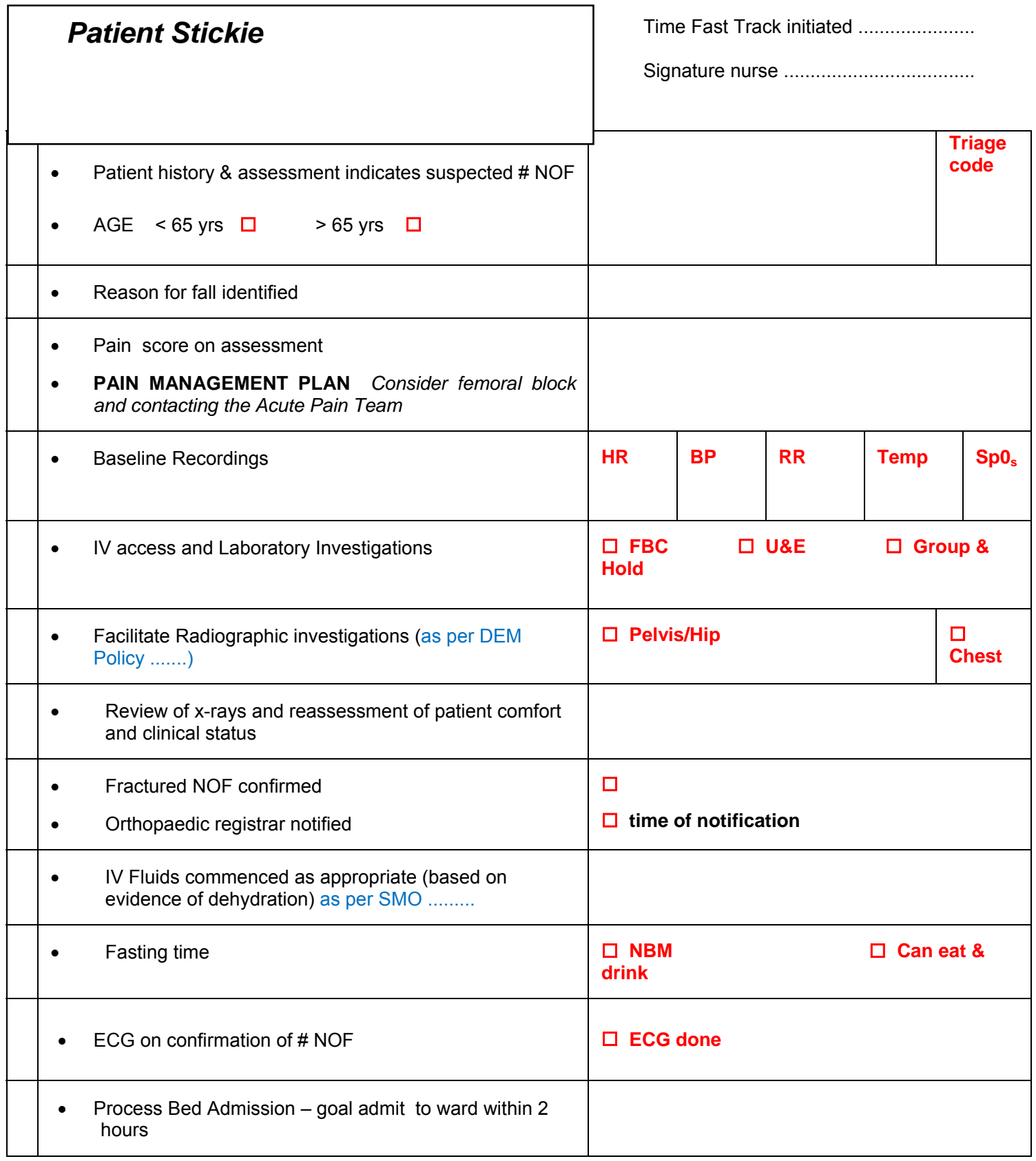




\section{Appendix E}

Adult vital signs chart (sections for respirations, oxygen saturation, blood pressure, pulse temperature, urine rate, level of consciousness and pain score $)(\mathrm{CCDHB})$

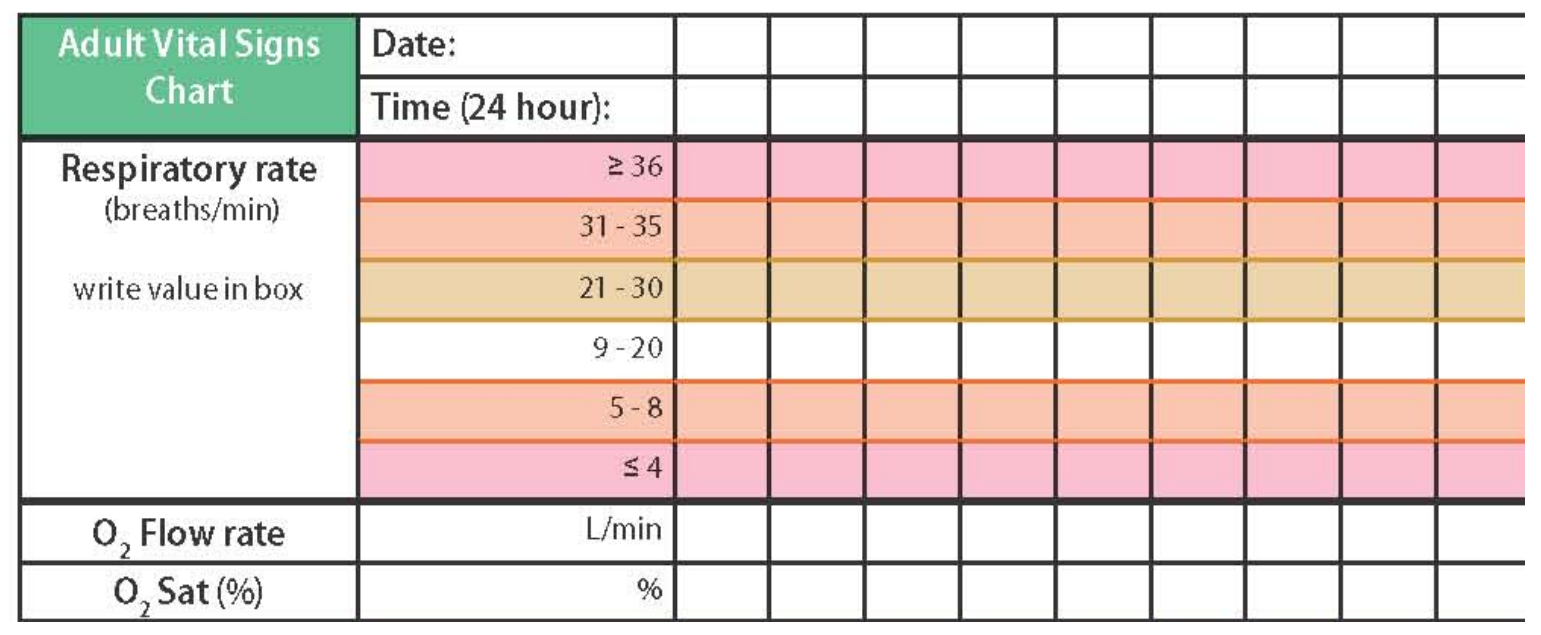

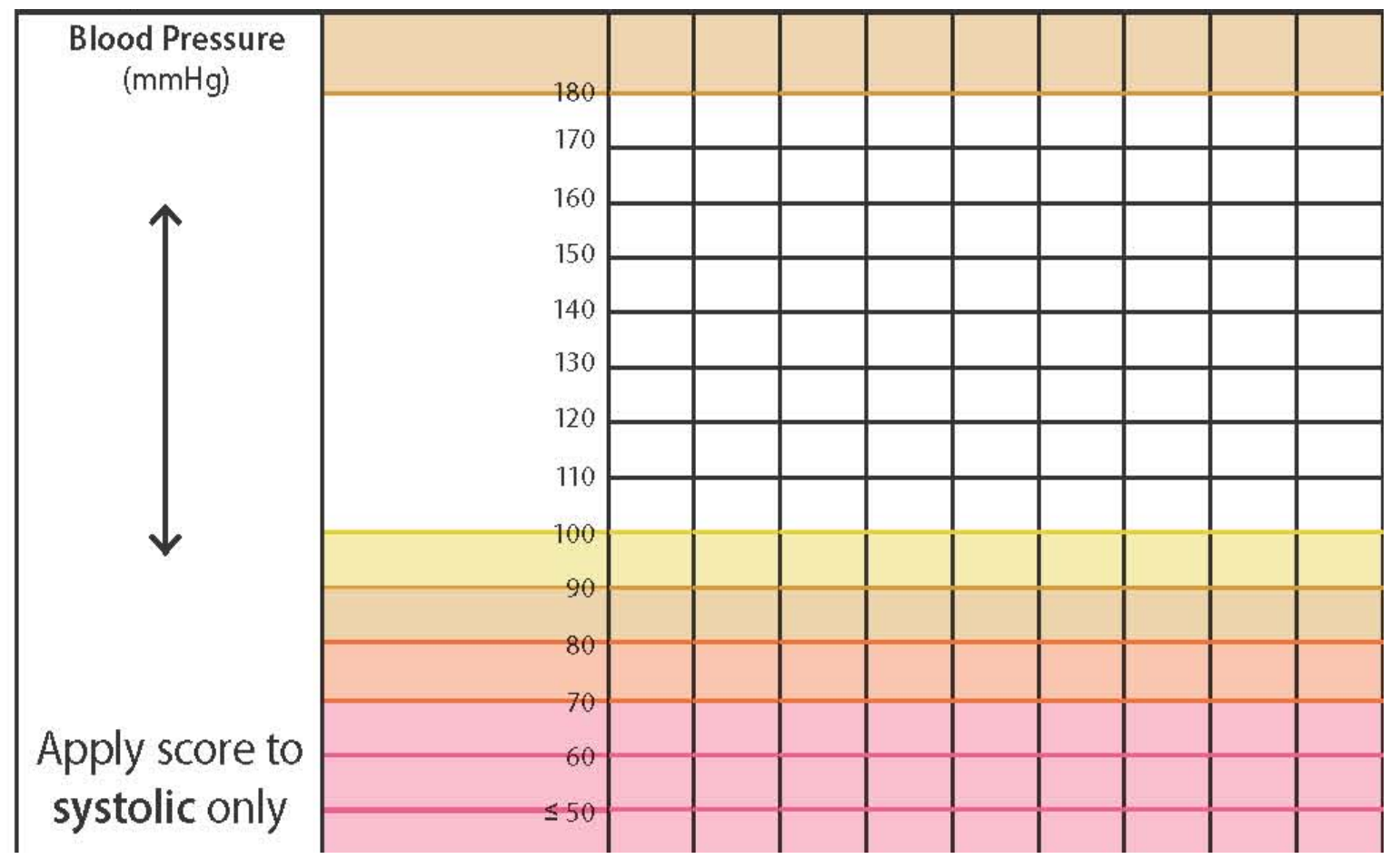




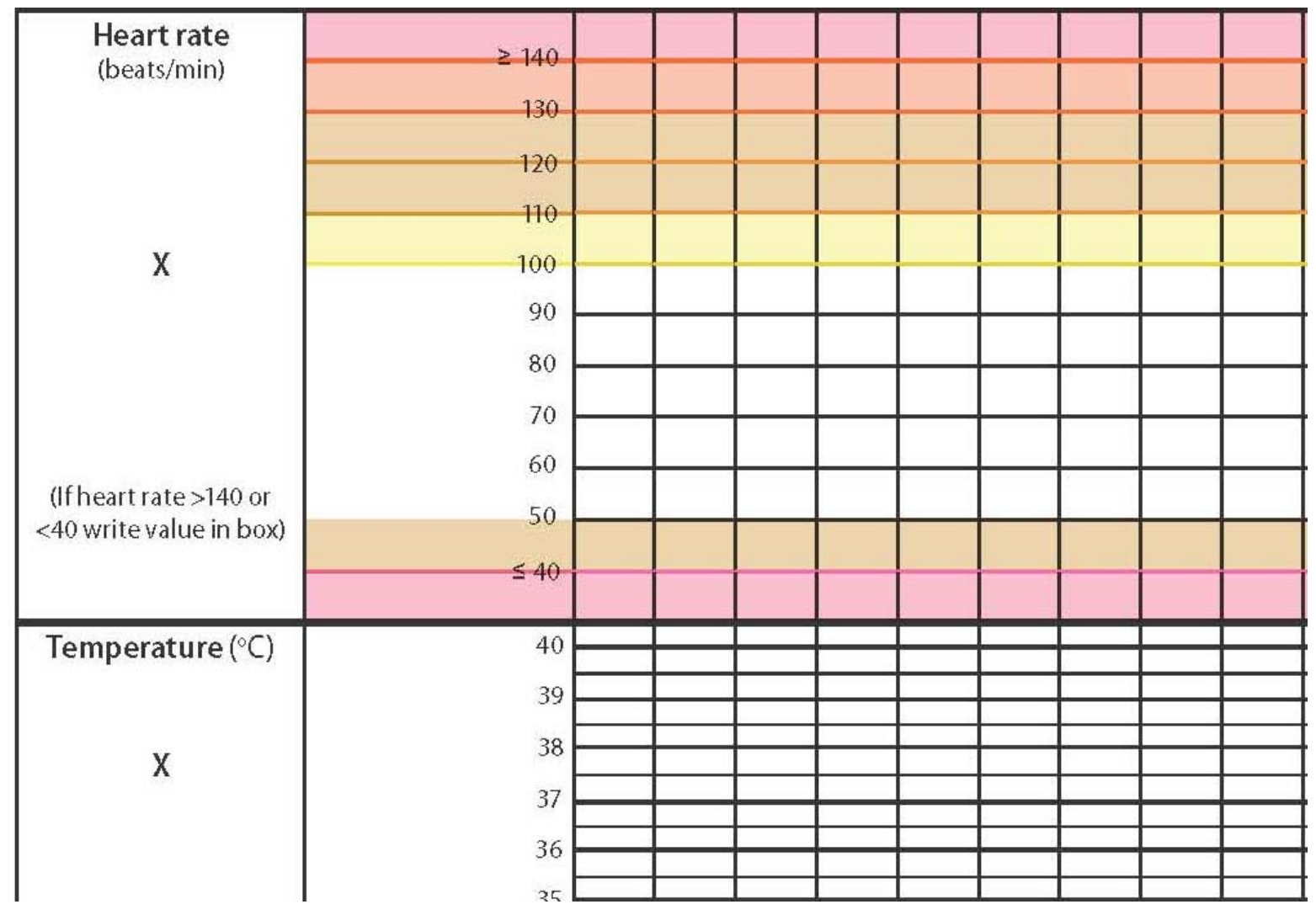

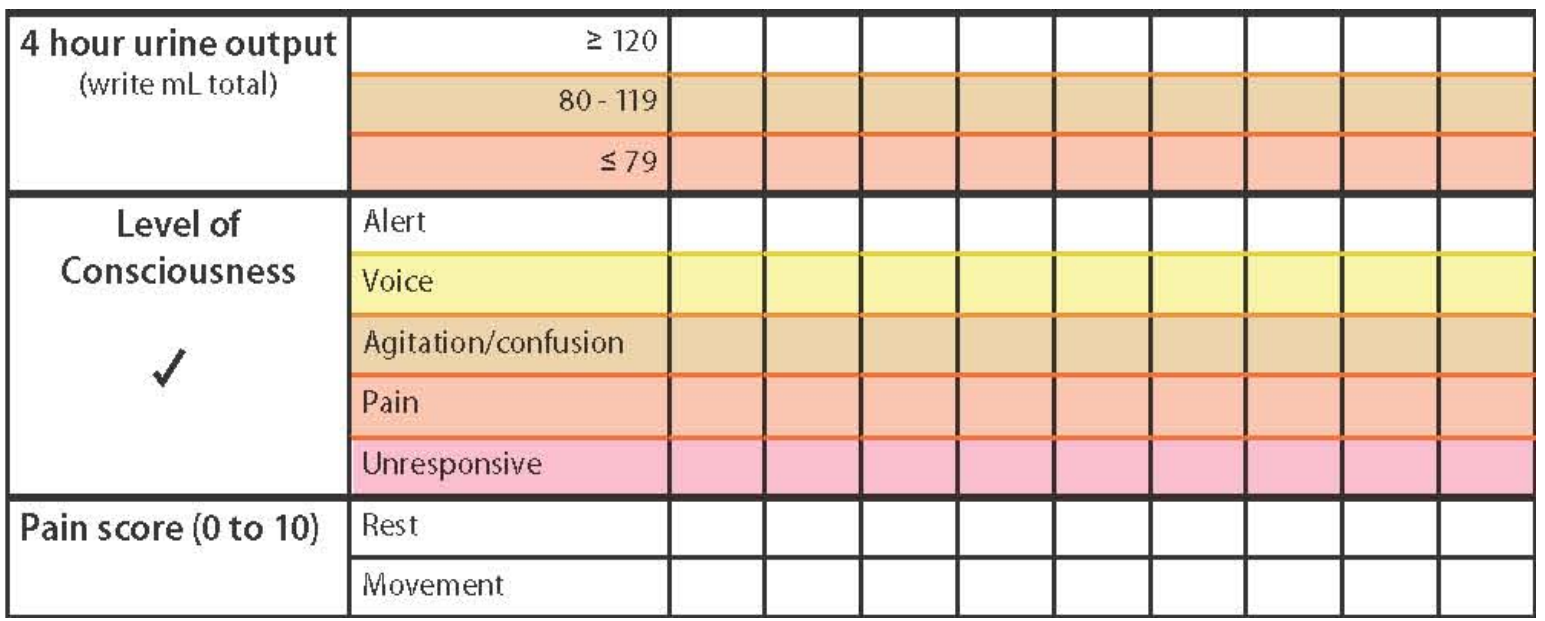


Appendix F

Expedited Review of Observational Studies ethical application

\section{Expedited Review of Observational Studies Application Form}

References in this form to the Guidelines are to the Ethical Guidelines for Observational Studies:

Observational research, audits and related activities, National Ethics Advisory Committee, December 2006. Investigators applying for expedited review of low-risk observational research, or of an audit or other activity related to observational research, must have read these Guidelines.

Part A: General - is expedited review appropriate or required for this study?

\begin{tabular}{|c|c|c|c|}
\hline 1 & ntle of project & \multicolumn{2}{|c|}{$\begin{array}{l}\text { Relationships of preoperative fluid management of geriatric patients admitted with hip fracture } \\
\text { and perioperative patient outcomes of reported confusion, and postoperative intravenous fluic } \\
\text { requirement and length of stay. }\end{array}$} \\
\hline \multirow[t]{9}{*}{2} & \multicolumn{3}{|l|}{ Investlgators } \\
\hline & \multirow[t]{8}{*}{ a) Princlpal Investigator } & \multirow{2}{*}{$\begin{array}{l}\text { Name: } \\
\text { Position: }\end{array}$} & Victoria C Ward \\
\hline & & & Staff Nurse, CCDHB, Wellington Hospital Campus \\
\hline & & \multicolumn{2}{|c|}{ Contact details } \\
\hline & & \multirow{3}{*}{$\begin{array}{l}\text { Address: } \\
\text { Phone: } \\
\text { Email: }\end{array}$} & 56a Duncan Tce, Kilbimie, Wellington 6022 \\
\hline & & & 045502958 \\
\hline & & & vickiward@orcon.net.nz \\
\hline & & \multicolumn{2}{|c|}{ Brief statement of relevant experience } \\
\hline & & & $\begin{array}{l}\text { Postgraduate diploma of nursing (2010) } \\
\text { Clinical research coordinator - Otago Medical School (2010 -11) }\end{array}$ \\
\hline & \multirow{6}{*}{$\begin{array}{l}\text { b) Supervisor (ff student } \\
\text { researcher) }\end{array}$} & \multirow{6}{*}{$\begin{array}{l}\text { Name: } \\
\text { Position: } \\
\text { Contact d } \\
\text { Address: } \\
\text { Phone: } \\
\text { Email: }\end{array}$} & Elaine Dennison \\
\hline & & & Professor of Clinical Research \\
\hline & & & talls \\
\hline & & & Victoria University, PO Box 600 , Wellington 6140 \\
\hline & & & 044635233 \\
\hline & & & elaine.dennison@vuw.ac.nz \\
\hline & \multirow{6}{*}{$\begin{array}{l}\text { c) Co-Investlontoris (include } \\
\text { contact detalls for each } \\
\text { co-investigator) }\end{array}$} & \multirow{6}{*}{$\begin{array}{l}\text { Name: } \\
\text { Position: } \\
\text { Contact de } \\
\text { Address: } \\
\text { Phone: } \\
\text { Email: }\end{array}$} & \\
\hline & & & \\
\hline & & & atalls \\
\hline & & & \\
\hline & & & \\
\hline & & & \\
\hline
\end{tabular}




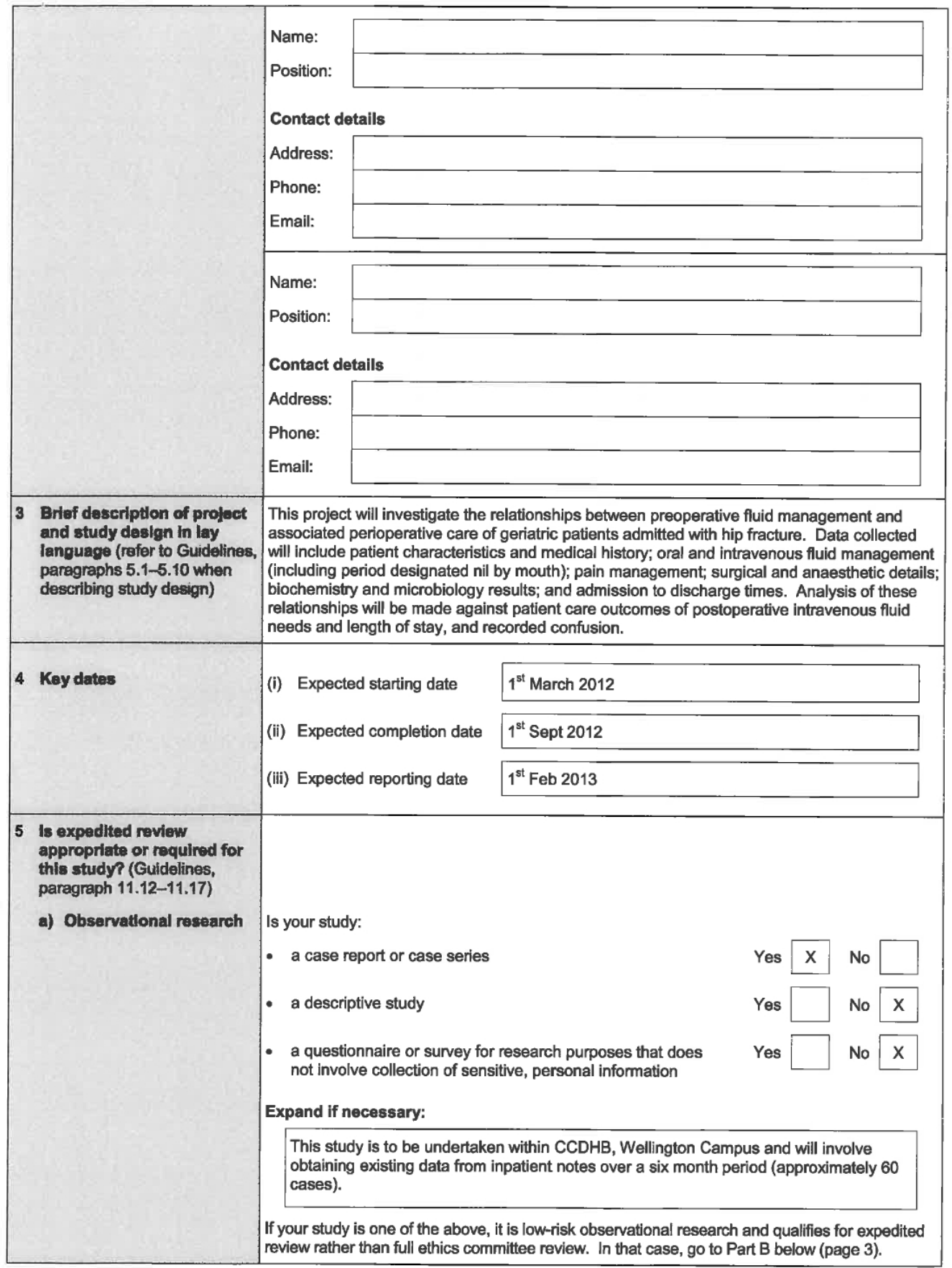




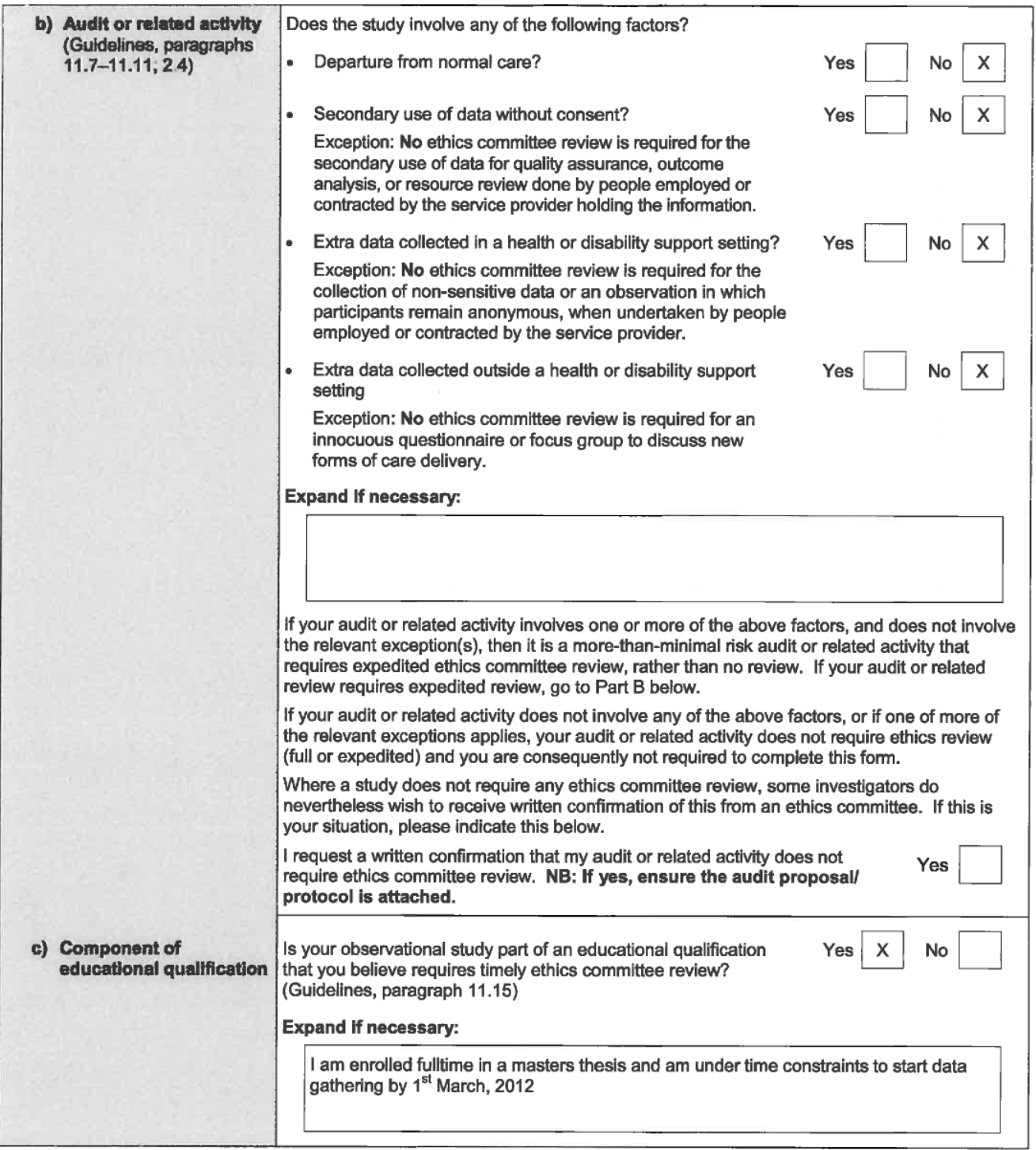




\section{Part B: Expedited review}

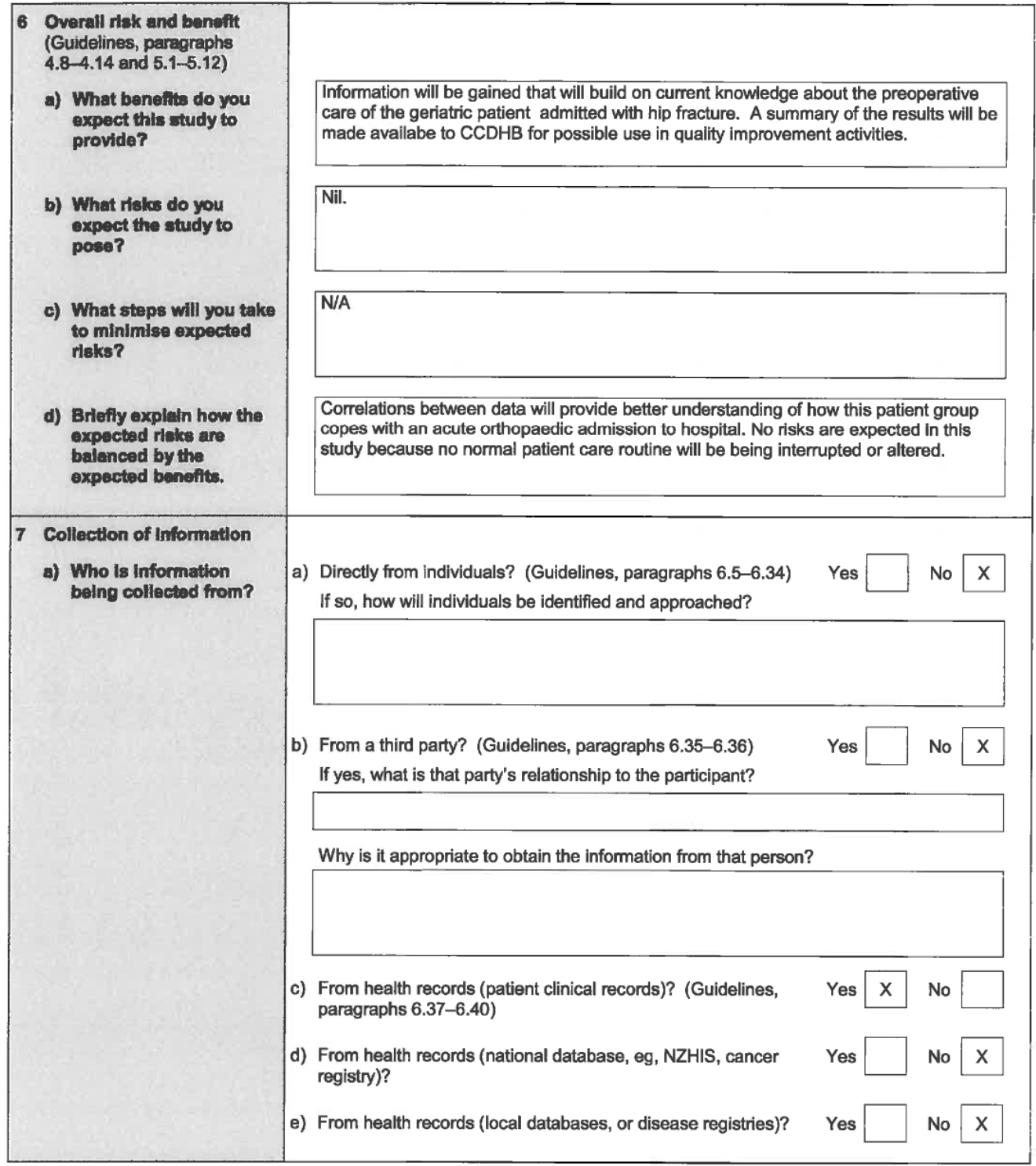




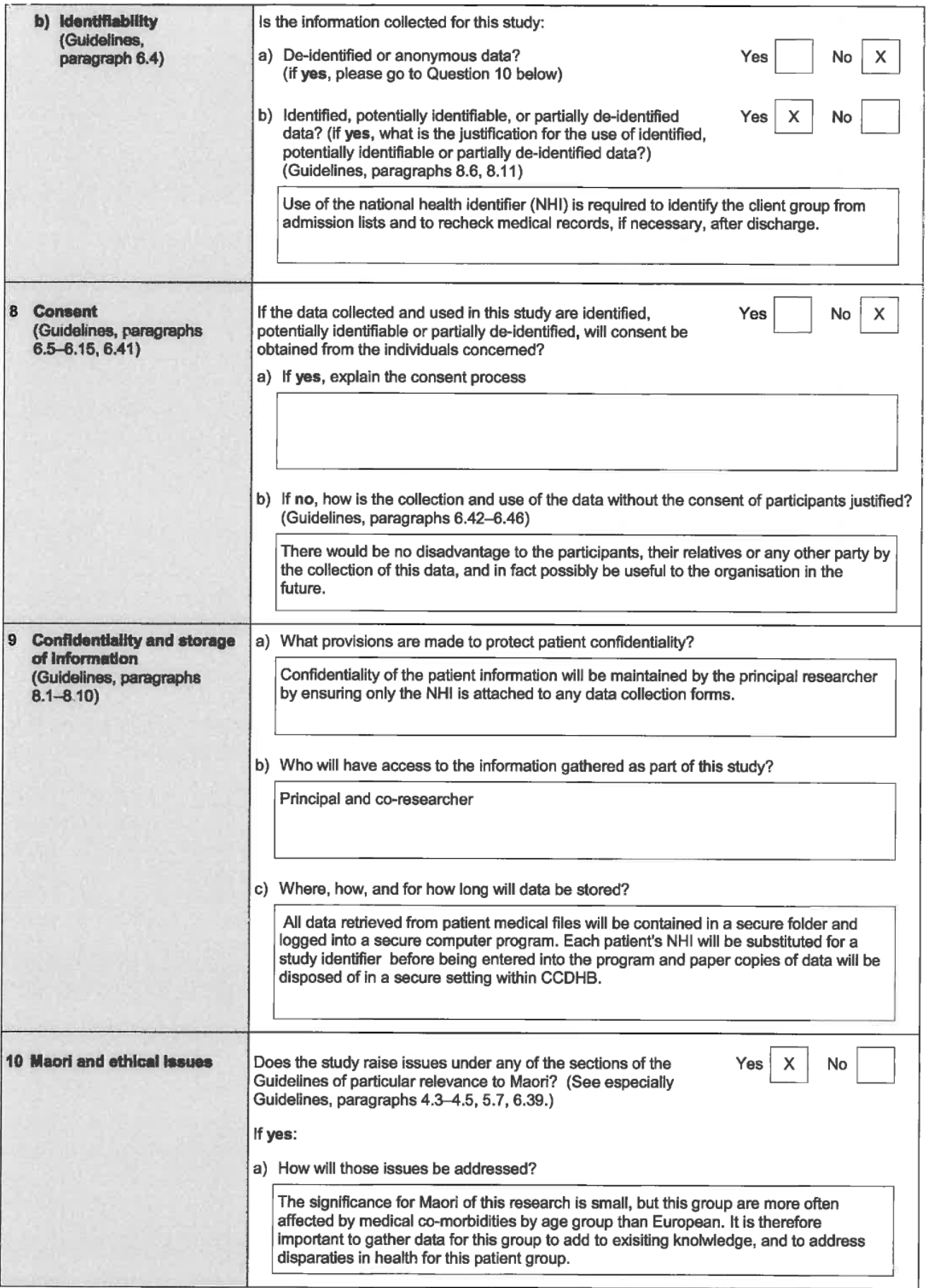




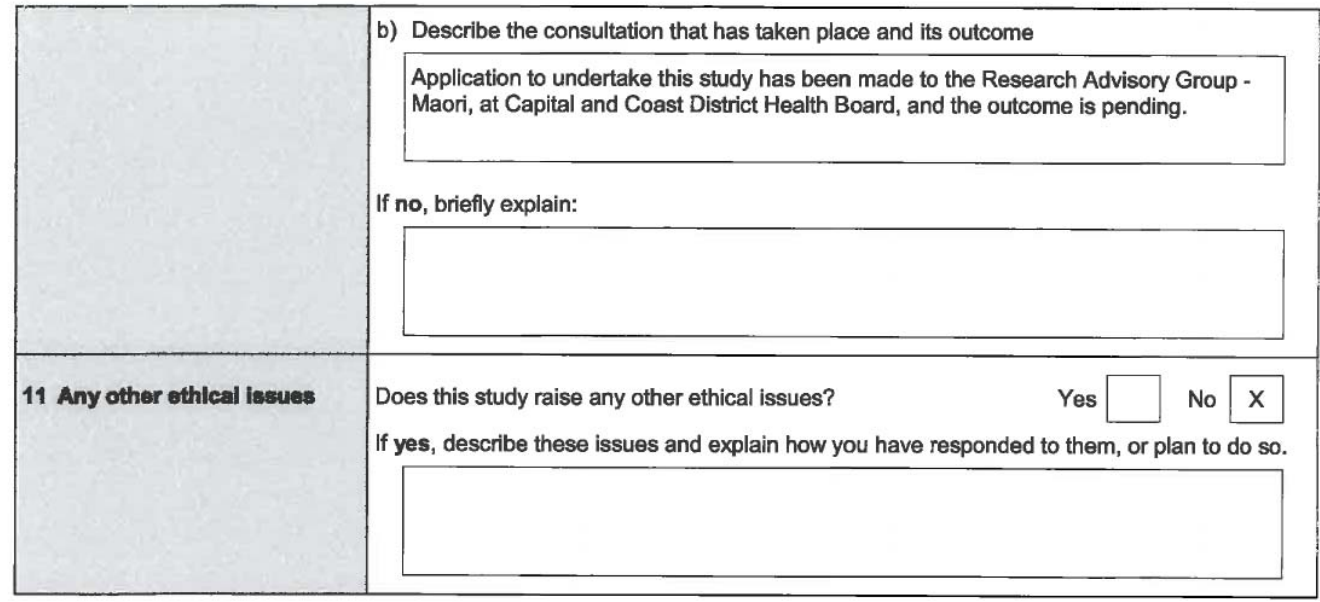

\section{Declaration by Principal Investigator}

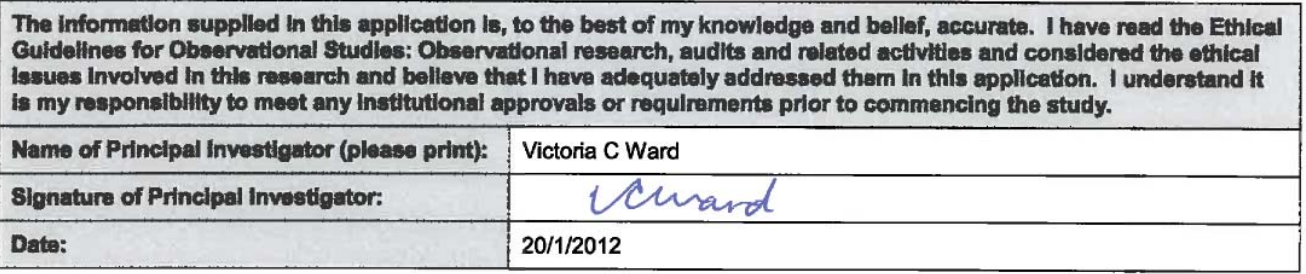

\section{Declaration by Supervisor}

\begin{tabular}{|c|c|c|c|}
\hline \multirow{4}{*}{$\begin{array}{l}\text { I have revlewed the af } \\
\text { audits and related act } \\
\text { application. I take ove } \\
\text { addreseed prtor to its } \\
\text { Name (please print): } \\
\text { Signature: } \\
\text { Date: }\end{array}$} & \multirow{4}{*}{$\begin{array}{l}\text { plication and read the Ethical Guldelin } \\
\text { vities. I confirm that the student has } \\
\text { rall responsiblilty for the study Includ } \\
\text { commencement. } \\
\text { Elaine Dennison } \\
\text { 20/1/2012 }\end{array}$} & \multirow{4}{*}{$\begin{array}{l}\text { nes for Observa } \\
\text { considered the } \\
\text { ing ensuring al } \\
\text { Institution: } \\
\text { Designation: } \\
\end{array}$} & $\begin{array}{l}\text { nal Studles: Observational } \\
\text { Ical lssues and accurately } \\
\text { stitutional requirements h }\end{array}$ \\
\hline & & & \\
\hline & & & Victoria University \\
\hline & & & Professor of Clinical Research \\
\hline
\end{tabular}

\section{Documents checklist}

\begin{tabular}{|l|c|}
\hline Doeument & Attached \\
\hline Study protocol & Yes \\
\hline Questionnaire or data collection form & Yes \\
\hline Information sheet/s (if required) & N/A \\
\hline Consent form/s (if required) & N/A \\
\hline $\begin{array}{l}\text { Any other information to be provided to participants (eg, letters } \\
\text { of invitation, advertisements) }\end{array}$ & N/A \\
\hline
\end{tabular}




\section{Appendix G}

\section{Expedited Review of Observational Studies ethical approval}

Health
and
Disability
Ethics
Committees

13 February 2012

Victoria C Ward

56a Duncan Terrace

Kilbirnie

Wellington 6022

Dear Victoria C Ward

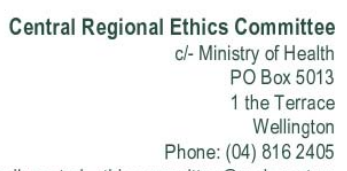

Email: central_ethicscommittee@moh.govt.nz

\section{Re: Ethics ref:} Study title:

CEN/12/EXP/009 (please quote in all correspondence)

Relationships of preoperative fluid management of geriatric patients admitted with hip fracture and perioperative patient outcomes of reported confusion, and postoperative intravenous fluid requirement and length of stay.

Investigators:

This expedited study was given ethical approval by the Chairperson of the Central Region Ethics Committee on 3 February 2012.

\section{Approved Documents}

- Expedited Review of Observational Studies (for the above study)

This approval is valid until 01/01/2013 provided that Annual Progress Reports are submitted (see below)

\section{Annual Progress Reports and Final Reports}

The first Annual Progress Report for this study is due on the $03 / 02 / 2013$. The Annual Report Form that should be used is available at www.ethicscommittees.health.govt.nz. Please note that if you do not provide a progress report by this date, ethical approval may be withdrawn

A Final Report is also required at the conclusion of the study. The Final Report Form is also available at www.ethicscommittees.health.govt.nz.

We wish you all the best with your study.

Please do not hesitate to contact me should you have any queries.

Yours sincerely

$44 \cot$

Sonia Scott

Administrator

Central Regional Ethics Committee 
Appendix $\mathrm{H}$

Research Advisory Group - Maori ethical approval

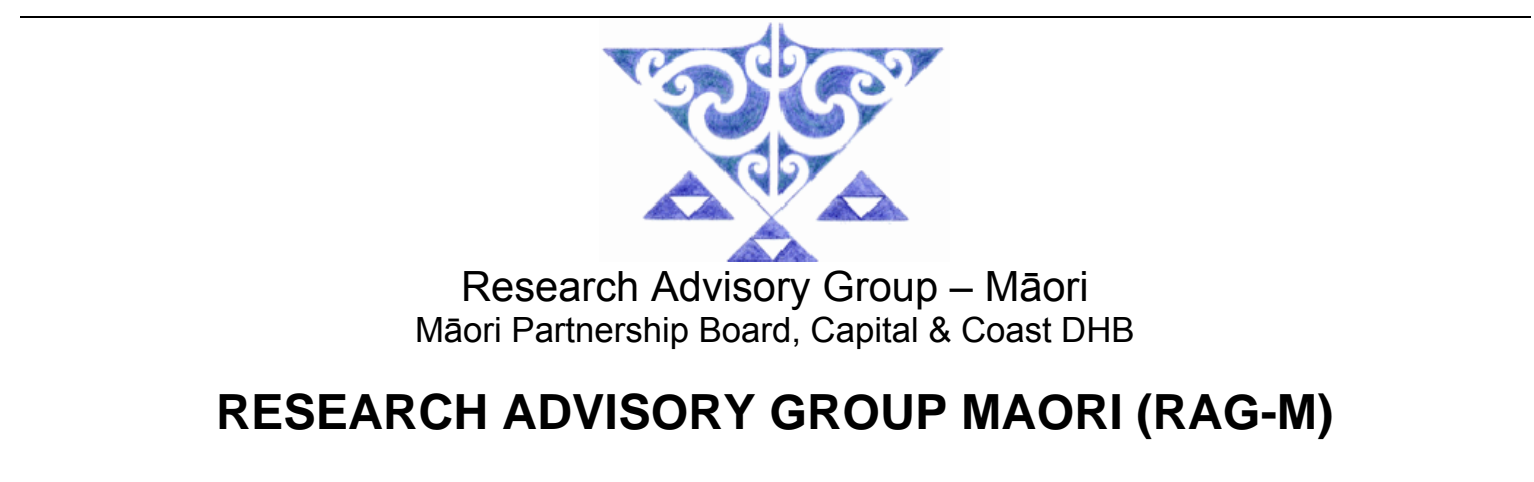

04 April 2012

Victoria C. Ward

56a Duncan Terrace

Kilbirnie

Wellington 6022

Tena koe

\section{RAG-M 2012/172 - Final Letter of Endorsement}

On behalf of the Research Advisory Group Māori I write in relation to your study entitled "Investigation of the relationships between preoperative fluid management of geriactric patients admitted with hip fracture and perioperative patient outcomes of reported confusion and postoperative intravenous fluid requirements and length of stay. Short title: Impact of altered hydration status on geriatric patients admitted acutely for surgery".

RAG-M are satisfied that you have read and understood the expectations outlined in this letter. We have received confirmation acknowledging and addressing these expectations and understand you are awaiting reply from Whanau Care Services, therefore RAGM are happy to endorse this research project.

Given the possibility of Māori patients as participants, we would expect that:

- you would ensure that any Mãori who participates is well informed and supported, including their whanau as appropriate; 
- you would recognise any cultural expectations and seek to meet these expectations responsively;

- treat any blood and tissue samples taken consistently with the CCDHB policy on human samples which is endorsed by this Committee.

We would be interested in receiving a summary of the results of your study, and we would be interested to find out how many Māori participants were recruited.

RAG-M has assessed your proposal from a Maori cultural, clinical and community perspective and we wish you well with this study.

Although the number of Maori involved is likely to be small we acknowledge the incidence of co-morbidities compared with European patients and thus the importance of data study and patient-care outcomes.

We would be interested to find out how many Māori participants were recruited.

RAG-M supports your application and we look forward to the results of your research and to their provision to Capital and Coast District Health Board for possible use in QI activities.

Nāku noa nā

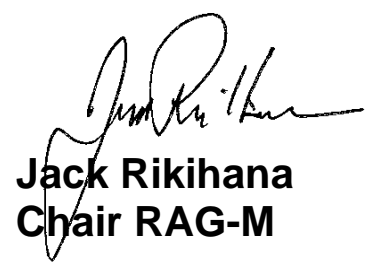




\section{Appendix I}

\section{Data collection form version 8}

Thesis data collection form - V Ward 2012

\begin{tabular}{|c|c|c|c|c|}
\hline & & & Participant & \\
\hline & Reference & Data to gather & Data entered & \\
\hline 1 & Date of birth & $00 / 00 / 0000$ & & \\
\hline 2 & Gender & 1. Male 2.Female & & \\
\hline 3 & Domesticity & $\begin{array}{l}\text { 1. home 2. res. care } \\
\text { 3. hospital/hospice }\end{array}$ & & \\
\hline 4 & Ethnicity & as per $\mathrm{CCDHB}$ & & \\
\hline 5 & Admit A + E & Date & 0000 & $\mathrm{hr}$ \\
\hline 6 & $\begin{array}{l}\text { Observations } \\
-\mathbf{A}+\mathbf{E} \text { admission }\end{array}$ & $\begin{array}{l}\mathrm{T} \\
\mathrm{P} \\
\mathrm{R}\end{array}$ & $\begin{array}{l}\mathrm{BP} \text { (systolic) } \\
\mathrm{O}_{2} \\
\mathrm{Hb}\end{array}$ & \\
\hline 7 & $\begin{array}{l}\text { Observations } \\
-\quad 48 h r s \text { postop }\end{array}$ & $\begin{array}{l}\mathrm{T} \\
\mathrm{P} \\
\mathrm{R} \\
\end{array}$ & $\begin{array}{l}\mathrm{BP} \text { (systolic) } \\
\mathrm{O}_{2} \\
\mathrm{Hb}\end{array}$ & \\
\hline 8 & $\begin{array}{l}\text { Biochemistry } \\
\text { - on admission }\end{array}$ & $\mathrm{Na}$ & $\begin{array}{l}\text { GFR } \\
\text { Alb } \\
\text { WCC }\end{array}$ & 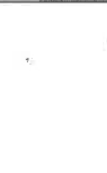 \\
\hline 9 & $\begin{array}{l}\text { Biochemistry } \\
24 \text { - 48hrs postop }\end{array}$ & $\begin{array}{l}\mathrm{Na} \\
\mathrm{K} \\
\mathrm{Cr}\end{array}$ & $\begin{array}{l}\text { GFR } \\
\text { Alb } \\
\text { WCC }\end{array}$ & \\
\hline 10 & Hx co-omorbidities & $\begin{array}{l}\text { 1. Renal } \\
\text { 2. Cardiac } \\
\text { 3. Pulmonary } \\
\text { 4. Vascular } \\
\text { 5. Diabetes } \\
\text { 6. Dementia } \\
\text { 7. Smoker } \\
\text { 8. Alcoholic }\end{array}$ & & \\
\hline 11 & $\begin{array}{l}\text { Medications on } \\
\text { admission }\end{array}$ & Name & Type & \\
\hline 12 & Delay to OT stated & & & \\
\hline 13 & Preop Oxygen & 1. Yes & & \\
\hline 14 & $\begin{array}{l}\text { Preop pain score } \\
\text { (moving) }\end{array}$ & $1-10$ & & \\
\hline
\end{tabular}

Data collection form - V Ward Feb 2012 - version 8 


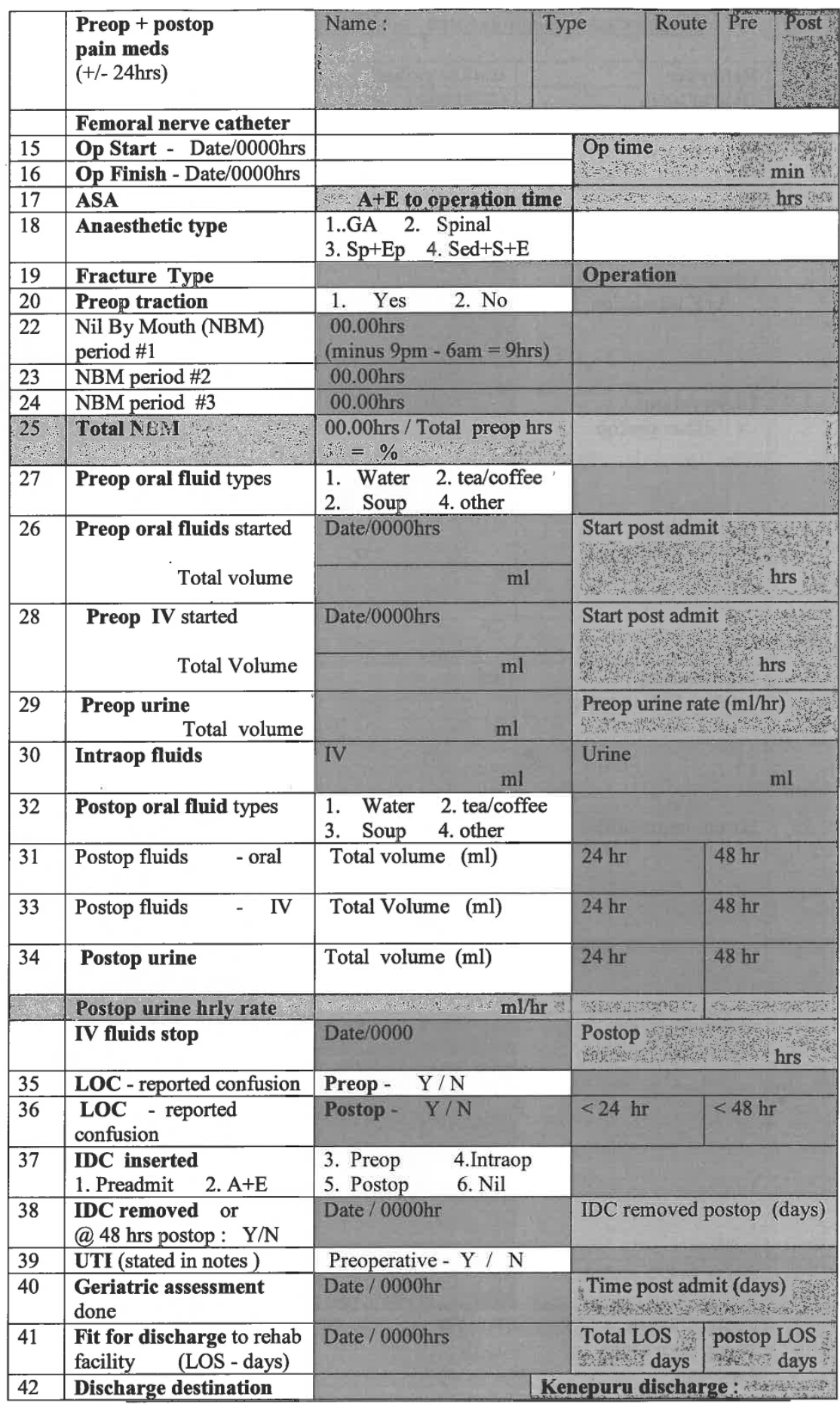

Data collection form - V Ward Feb 2012 - version 8 


\section{Appendix J}

\section{Data collection form version 11}

Thesis data collection form - V Ward 2012

\begin{tabular}{|c|c|c|c|c|}
\hline & & & \multicolumn{2}{|l|}{ Participant ID } \\
\hline & Reference & Data to gather & Data entered & \\
\hline 1 & Date of birth & $00 / 00 / 0000$ & & \\
\hline 2 & Gender & 1. Male 2.Female & & \\
\hline 3 & Domesticity & & & \\
\hline 4 & Ethnicity & as per CCDHB & & \\
\hline 5 & Admit A + E & Date & 0000 & $\mathrm{hr}$ \\
\hline 6 & $\begin{array}{c}\text { Observations } \\
-\mathbf{A}+\mathbf{E} \text { admission } \\
\text { Urine: } \text { USG }= \\
\mathrm{pH}= \\
\text { protein }= \\
\end{array}$ & $\begin{array}{l}\mathrm{T} \\
\mathrm{P} \\
\mathrm{R} \\
\end{array}$ & $\begin{array}{l}\mathrm{BP} \text { (systolic) } \\
\mathrm{O}_{2} \\
\mathrm{Hb}\end{array}$ & \\
\hline 7 & $\begin{array}{l}\text { Observations } \\
-\quad \text { 48hrs postop } \\
\text { 24hr postop } \mathrm{Hb}\end{array}$ & $\begin{array}{l}\mathrm{T} \\
\mathrm{P} \\
\mathrm{R}\end{array}$ & $\begin{array}{l}\mathrm{BP} \text { (systolic) } \\
\mathrm{O}_{2} \\
\mathrm{Hb}\end{array}$ & \\
\hline 8 & $\begin{array}{l}\text { Biochemistry } \\
\text { - on admission }\end{array}$ & $\begin{array}{l}\mathrm{Na} \\
\mathrm{K} \\
\mathrm{Cr}\end{array}$ & $\begin{array}{l}\text { GFR } \\
\text { Alb } \\
\text { WCC }\end{array}$ & \\
\hline 9 & \begin{tabular}{|} 
Biochemistry \\
$24-48 h r s$ postop
\end{tabular} & $\begin{array}{l}\mathrm{Na} \\
\mathrm{K} \\
\mathrm{Cr}\end{array}$ & $\begin{array}{l}\text { GFR } \\
\text { Alb } \\
\text { WCC }\end{array}$ & \\
\hline 10 & Hx co-omorbidities & & & \\
\hline 11 & $\begin{array}{l}\text { Medications on } \\
\text { admission }\end{array}$ & Name & Type & \\
\hline 12 & Delay to OT stated & & $\begin{array}{l}\text { Delay before } \\
\text { admission: } \mathrm{Y} / \mathrm{N}\end{array}$ & \\
\hline 13 & Preop Oxygen & 1. Yes & & \\
\hline 14 & $\begin{array}{l}\text { Preop pain score } \\
\text { (on movement) }\end{array}$ & $0-10$ & & \\
\hline
\end{tabular}

Data collection form - V Ward Feb 2012 - version 11 


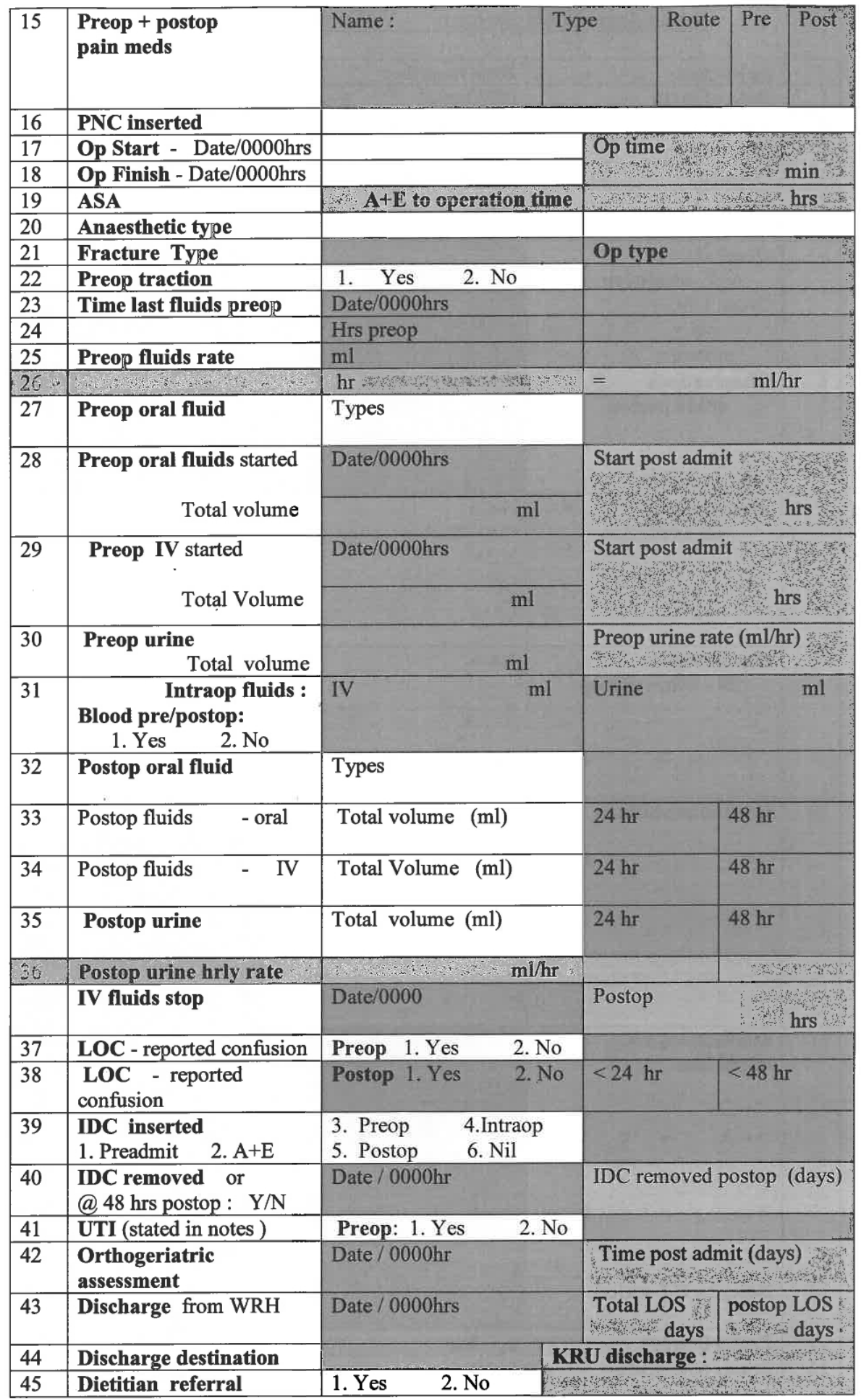




\section{Appendix K}

Data analysis grid

\begin{tabular}{|c|c|c|c|c|c|c|}
\hline Reference & Data source & Data entered & Data type & Variable type & & \\
\hline ID & & $1-100$ & Identifier & Demographic & Exposure & Outcome \\
\hline DOB & E-Medical record & $00 / 00 / 0000$ & Continuous variable & & & \\
\hline Age & E-Medical record & $\begin{array}{l}00.0 \text { years (measure in } \\
\text { year quarters) }\end{array}$ & Continuous variable & & & \\
\hline Gender & E-Medical record & $\begin{array}{l}\text { Male } \\
\text { Female }\end{array}$ & Discrete variable & & & \\
\hline Domesticity & $\begin{array}{l}\text { E-Medical record } \\
\text { - ED notes }\end{array}$ & $\begin{array}{l}\text { own home alone } \\
\text { own home + spouse own } \\
\text { home + family } \\
\text { residential care } \\
\text { hospital/hospice } \\
\text { dementia unit } \\
\text { Homeless } \\
\text { Kenepuru Rehab unit } \\
\text { CCDHB Wgtn }\end{array}$ & Discrete variable & & & \\
\hline $\begin{array}{ll}\text { Ethnicity } \\
- & \text { categories as per } \\
& \text { CCDHB } \\
- & \text { grouped as } \\
\text { indicated for } \\
\text { reporting }\end{array}$ & E-Medical record & $\begin{array}{l}\text { NZ European } \\
\text { European } \\
\text { Other European } \\
\text { NZ Maori } \\
\text { Samoan } \\
\text { Cook Island Maori } \\
\text { Tongan }\end{array}$ & Discrete variable & & & \\
\hline
\end{tabular}




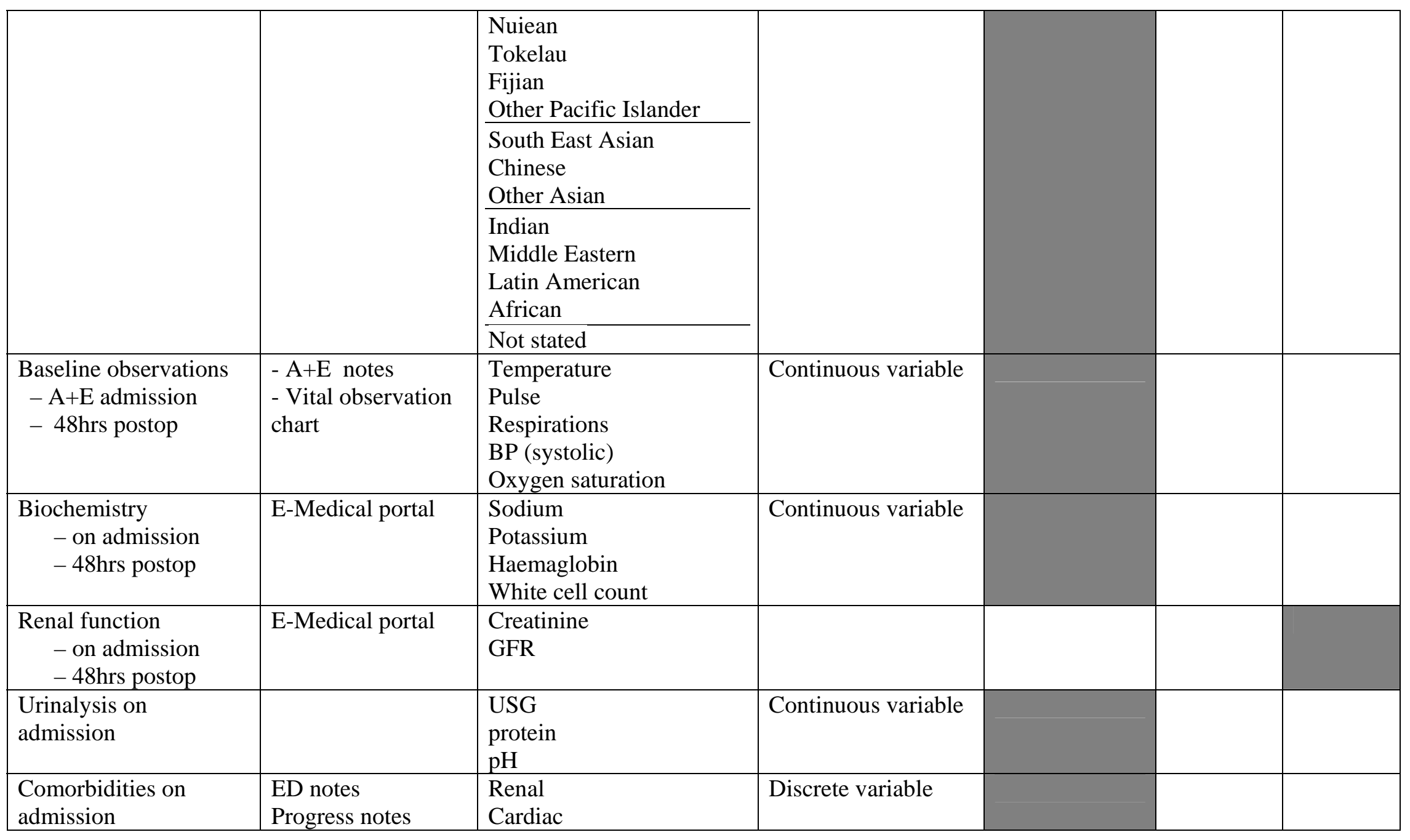




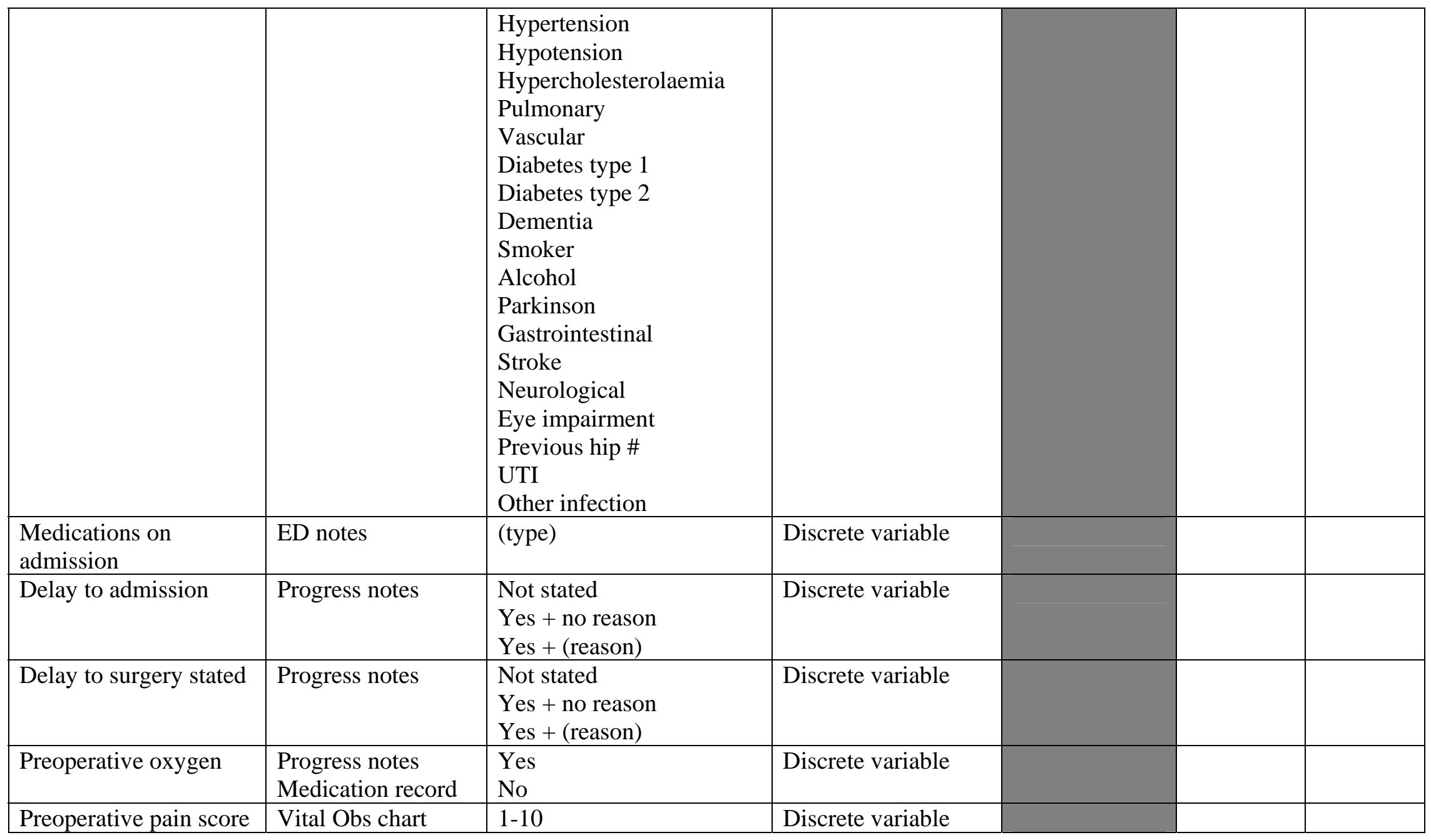




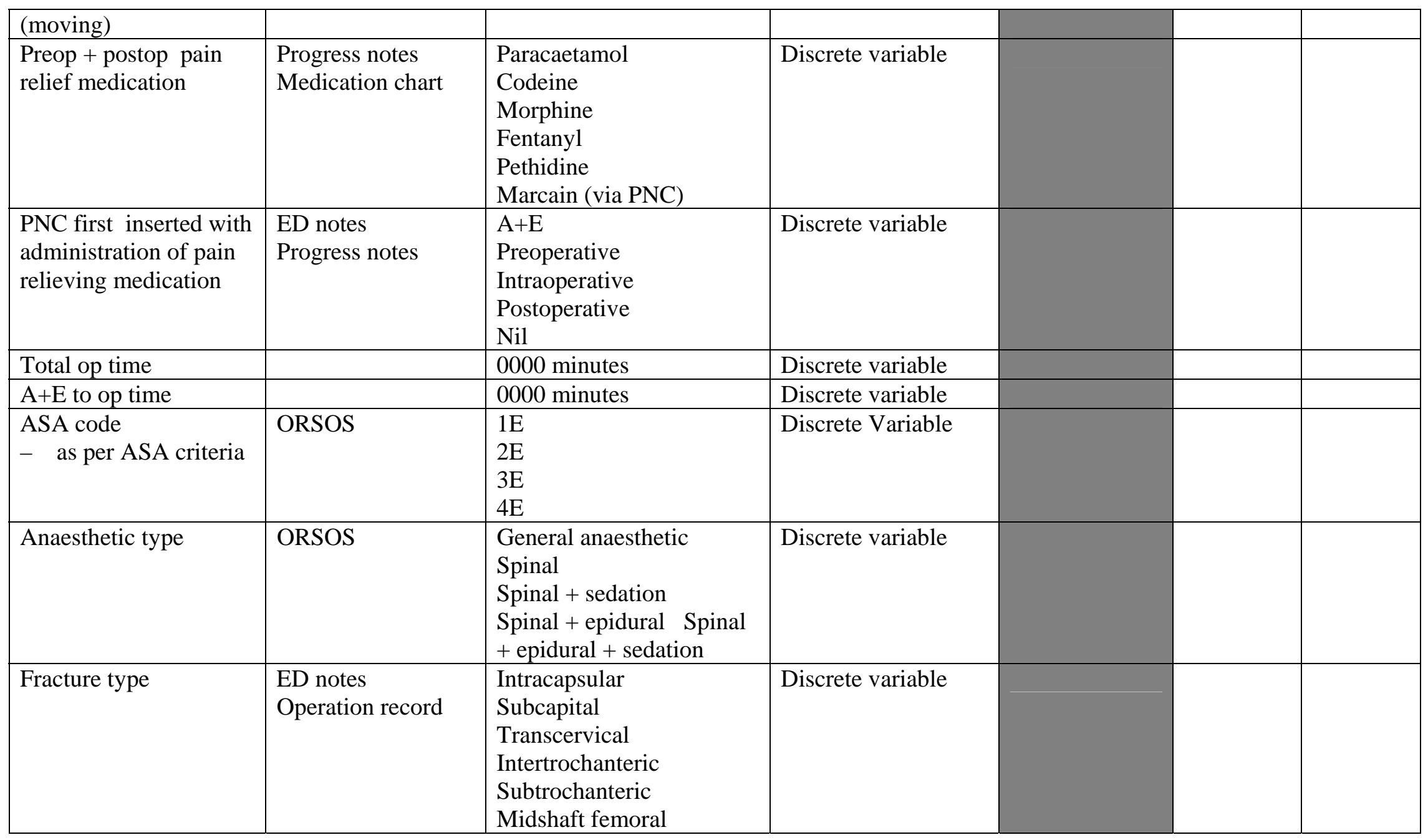




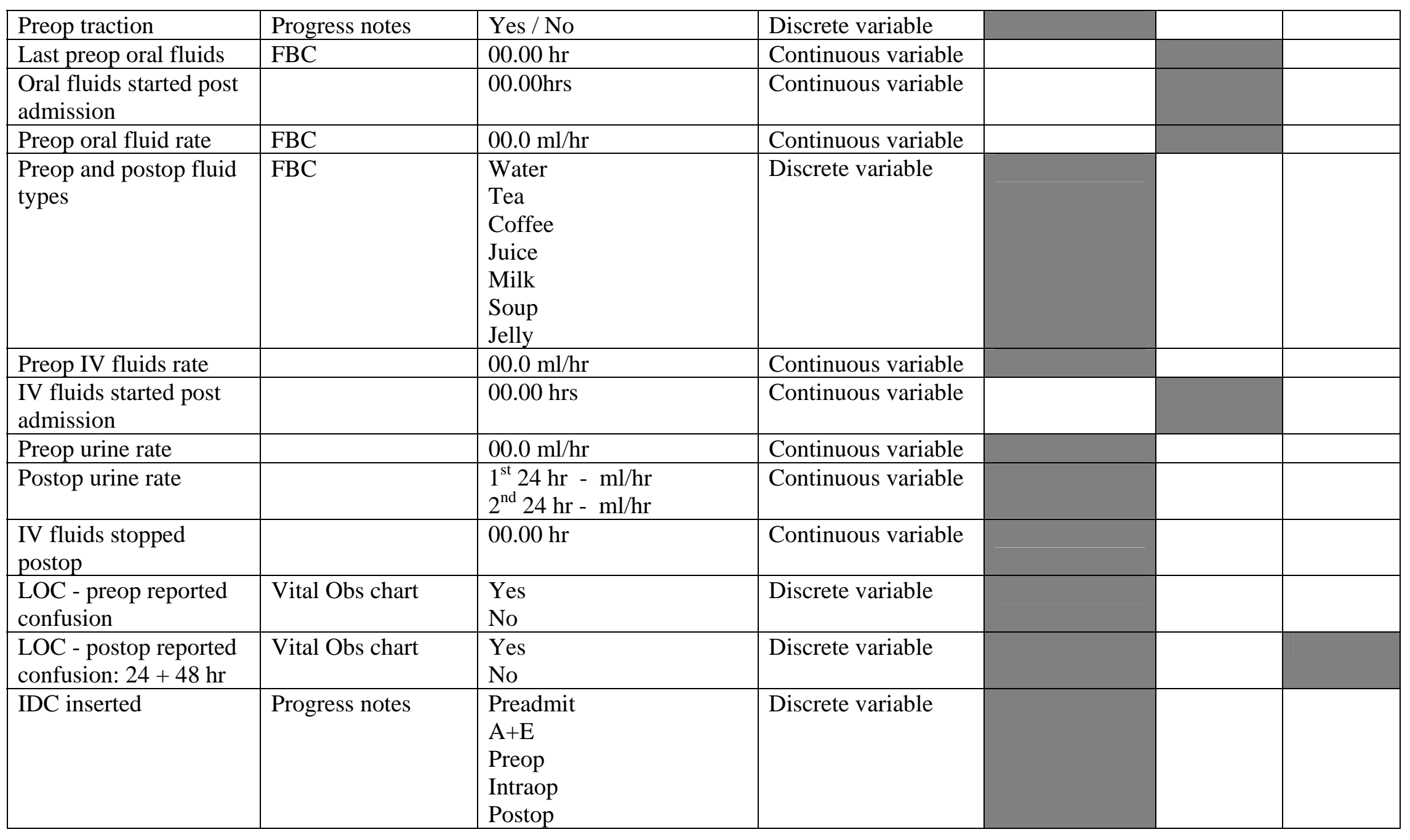




\begin{tabular}{|l|l|l|l|l|l|}
\hline & & Nil & & \\
\hline $\begin{array}{l}\text { IDC insitu @ 48hr } \\
\text { postop }\end{array}$ & $\begin{array}{l}\text { Progress notes } \\
\text { FBC }\end{array}$ & $\begin{array}{l}\text { Yes } \\
\text { No }\end{array}$ & 00.0 days & Discrete variable & \\
\hline IDC total days insitu & & $\begin{array}{l}\text { Yes } \\
\text { No }\end{array}$ & Continuous variable & & \\
\hline UTI diagnosed preop & $\begin{array}{l}\text { Progress notes } \\
\text { Microbiology }\end{array}$ & 00.0 days & Continuous variable & & \\
\hline $\begin{array}{l}\text { Admit to orthogeriatric } \\
\text { assessment }\end{array}$ & Progress notes & $\begin{array}{l}\text { Kenepuru Rehab unit } \\
\text { own home alone } \\
\text { own home + spouse } \\
\text { own home + family } \\
\text { residential care } \\
\text { hospital/hospice } \\
\text { dementia unit } \\
\text { other rehab unit }\end{array}$ & Discrete variable & & \\
\hline Discharge destination & 00.0 days & Continuous variable & \\
\hline LOS postop & 00.0 days & Continuous variable & \\
\hline LOS KRU & & 00.0 days & Continuous variable & \\
\hline LOS Total CCDHB & & & \\
\hline
\end{tabular}




\begin{tabular}{|c|c|}
\hline Abbreviations & \\
\hline$A+E$ & Accident and Emergency \\
\hline ASA & Anaesthetic Society of America \\
\hline DOB & Date of birth \\
\hline FBC & Fluid balance chart \\
\hline GFR & glomerular filtration rate \\
\hline $\mathrm{Hr}$ & hour/s \\
\hline ID & participant identifier \\
\hline IDC & indwelling urinary catheter \\
\hline Intraop & intraoperative \\
\hline IV & intravenous \\
\hline KRU & Kenepuru rehab unit \\
\hline LOC & level of consciousness \\
\hline $\mathrm{ml} / \mathrm{hr}$ & millilitres per hour \\
\hline mins & minutes \\
\hline Op & operation \\
\hline ORSOS & theatre computing system \\
\hline PNC & peripheral nerve catheter \\
\hline Postop & postoperative \\
\hline Preop & preoperative \\
\hline Rehab & Rehabilitation \\
\hline UTI & urinary tract infection \\
\hline
\end{tabular}




\section{Appendix L}

Poster for staff

\section{Clinical Research Study}

\section{What is the relationship of preoperative fluid management in older adult patients admitted with hip fracture to postoperative outcomes?}

This study looks at the effect of fluid management of this patient group as it relates to the following postoperative outcomes:

o Renal function

o Reported new confusion

o Intravenous fluid requirements

o Length of stay in hospital

My background is in theatre nursing and I am undertaking this thesis project towards completion of the Masters of Clinical Research at Victoria University. I work in the orthopaedic specialty and have developed an interest in effectively managing the journey of the older adult patient with hip fracture through the perioperative environment.

This study hopes to provide information that will enhance management of this group of patients

at $\mathrm{CCDHB}$.

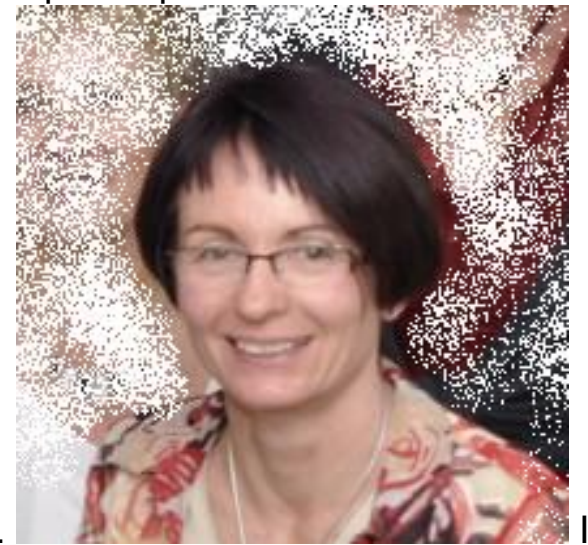

\section{st 2012 for approximately $6 \mathrm{~m}$, and also obtaining data from fluid balance} notes from $1^{\text {st }}$ March 2012 for approximately $6 \mathrm{mth}$, and also obtaining data from fluid balance charts, medication records, and the vital signs chart.

If you have any queries about this project or wish to know more please contact me or ask me when I am in your area. The Central Ethics Committee has approved this study, as has the CCDHB Research Advisory Group (Maori).

Thank you in anticipation of your support 
Reasons for measuring designated outcomes:

1. Renal function

- to ascertain level of kidney impairment (GFR)

- to measure how the body manages waste products (Creatinine)

2. Reported new confusion:

- confusion can be a symptom of a number of issues in the older adult patient

0 of lacking fluids or being dehydrated

0 of being in pain

0 of being out of the patient's normal environment

3. Postop IV fluids are usually required:

- to replace fluid loss due to not eating and drinking (fasting)

- replace blood loss due to the fracture or during surgery

- to be continued until patient is

o partaking of a near normal diet

o not confused

o recovered usual level of consciousness post anaesthesia

o taking oral pain relief

4. Postop length of stay is gauged by:

- ability to manage activities of daily living,

- reestablishment of preoperative psychosocial skills,

- physiotherapist and medical approval to rehab facility.

To assist with this study regular and legible documentation on fluid balance charts, vital observation charts, and the medication record are all that is required.

Your assistance in advising of any changes to expected discharge dates or destination for this group of patients would be much appreciated.

Thank you in anticipation for having me in your ward. 


\section{Appendix M}

\section{Sample (anonymous) patient data collection sheet}

Thesis data collection form -V Ward 2012

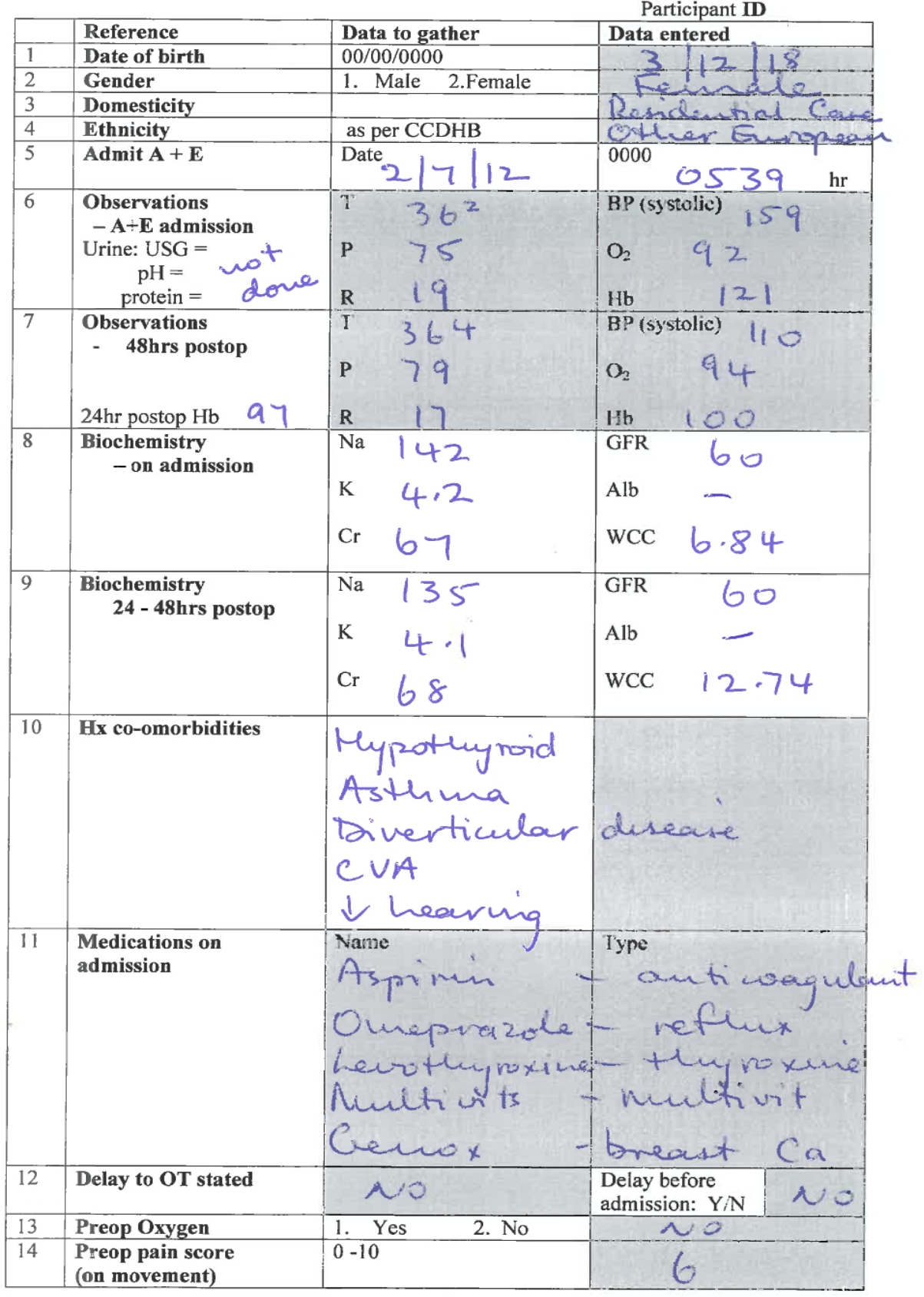

Data collection form - V Ward Feb 2012 - version 11 


\begin{tabular}{|c|c|c|c|}
\hline 15 & $\begin{array}{l}\text { Preop + postop } \\
\text { pain meds }\end{array}$ & $\begin{array}{l}\text { Name: } \\
\text { Treanalol } \\
\text { Sechedol } \\
\text { Pamadol }\end{array}$ & \begin{tabular}{|c|c|c|} 
Route & Pre & Pest \\
0 & & \\
0 & & \\
0 & & \\
\end{tabular} \\
\hline 16 & PNC inserted & nil & \\
\hline 17 & Op Start - Date/0000hrs & $3 / 7 / 12$ & \multirow{2}{*}{ Op time $79 \quad \min$} \\
\hline 18 & Op Finish - Date $/ 0000 \mathrm{hrs}$ & \multirow{2}{*}{$\frac{1222}{A+E \text { to operation time }}$} & \\
\hline 19 & ASA $\quad 3 E$ & & 30.0 \\
\hline 20 & Anaesthetic type & Epinal & \\
\hline 21 & Fracture Type & Subcomital & Optype He \\
\hline 22 & Preop traction & 1. Yes 2. No & $\triangle 0$ \\
\hline 23 & Time last fluids preop & Datei0000hrs & 112 \\
\hline 24 & & Hrs prcop & \\
\hline 25 & Preop fluids rate & $\mathrm{ml} \quad 380$ & \\
\hline 26 & & $\mathrm{hr} \quad 30$ & $=12.7 \mathrm{ml} / \mathrm{hr}$ \\
\hline 27 & Preop oral fluid & Types & $\mathrm{H}_{2} \mathrm{O}+\mathrm{CORRe}$ \\
\hline \multirow[t]{2}{*}{28} & \multirow{2}{*}{$\begin{array}{r}\text { Preop oral fluids started } \\
\text { Total volume }\end{array}$} & $\begin{array}{l}\text { Date/0000hrs } \\
2.17 .12\end{array}$ & \multirow{2}{*}{$\begin{array}{l}\text { Start post admit } \\
14.25 \text { hrs }\end{array}$} \\
\hline & & $380 \mathrm{ml}$ & \\
\hline \multirow[t]{2}{*}{29} & \multirow{2}{*}{$\begin{array}{l}\text { Preop IV started } \\
\text { Total Volume }\end{array}$} & $\begin{array}{l}\text { Dateogohrs } 0800 \\
2 / 2] 12\end{array}$ & \multirow{2}{*}{$\begin{array}{l}\text { Start post admit } \\
2.25 \text { hrs }\end{array}$} \\
\hline & & $2955 \mathrm{mi}$ & \\
\hline 30 & $\begin{array}{c}\text { Preop urine } \\
\text { Total volume }\end{array}$ & $1600 \mathrm{ml}$ & Preop urine rate $(\mathrm{ml} / \mathrm{hr})$ \\
\hline 31 & \begin{tabular}{|c|}
\multicolumn{2}{|c|}{ Intraop fluids : } \\
Blood pre/postop: \\
$\begin{array}{ll}\text { 1. Yes } & \text { 2. No } \\
\end{array}$
\end{tabular} & 2. 0 & Urine $18000 \mathrm{ml}^{\mathrm{ml}}$ \\
\hline 32 & Postop oral fluid & Types & $\begin{array}{l}\mathrm{H}_{20}+\mathrm{kis}+\mathrm{sour} \\
+ \text { fortisis }+0 \mathrm{I}\end{array}$ \\
\hline 33 & Postop fluids & Total volume $(\mathrm{ml})$ & \begin{tabular}{|c|c}
$24 \mathrm{hr}$ & $48 \mathrm{hr}$ \\
400 & 950
\end{tabular} \\
\hline 34 & Postop fluids & Total Volume (ml) & \begin{tabular}{rl|l}
$24 \mathrm{~h}$ & $88 \mathrm{~h}$ & \\
\end{tabular} \\
\hline 35 & Postop urine & Total volume $(\mathrm{ml})$ & \begin{tabular}{|c|c|c|}
$24 \mathrm{hr}$ & $48 \mathrm{hr}$ & $?$ \\
\end{tabular} \\
\hline 36 & Postop urine hrly rate & $\mathrm{ml} / \mathrm{hr}$ & $45+5$ \\
\hline & IV fluids stop & $\begin{array}{l}\text { Date' } 0100 \\
4(7 / 12 \quad 1500\end{array}$ & Postop $26.75 \mathrm{hrs}$ \\
\hline 37 & LOC - reported confusion & Preop 1. Yes & Tes \\
\hline 38 & $\begin{array}{l}\text { LOC - reported } \\
\text { confusion }\end{array}$ & Postop 1. Yes & $\begin{array}{r}-24 \mathrm{hr} \\
y e s\end{array}$ \\
\hline 39 & \begin{tabular}{|l|l|} 
IDC inserted \\
$\begin{array}{ll}\text { 1. Preadmit } & \text { 2. A+E } \\
\end{array}$
\end{tabular} & $\begin{array}{ll}\text { 3. Preop } & \text { 4.Intraop } \\
\text { 5. Postop } & \text { 6. Nil } \\
\end{array}$ & $A+E$ \\
\hline 40 & $\begin{array}{l}\text { IDC removed or } \\
\text { @ } 48 \text { hrs postop : Y } / \mathrm{N}\end{array}$ & 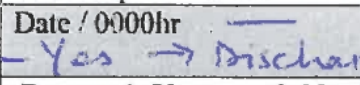 & $\begin{array}{l}\text { IDC removed postop (days) } \\
\text { ge. }\end{array}$ \\
\hline 41 & UTI (stated in notes ) & Preop: 1. Yes & $\triangle 0$ \\
\hline 42 & \begin{tabular}{|l|}
$\begin{array}{l}\text { Orthogeriatric } \\
\text { assessment }\end{array}$ \\
\end{tabular} & $\begin{array}{l}\text { Date } 10000 \mathrm{hr} \\
3 / 7112\end{array}$ & $\begin{array}{c}\text { Time post admit (days) } \\
1.2\end{array}$ \\
\hline 43 & Discharge from WRH & $\begin{array}{l}\text { Date / opoohrs } \\
6 / 7 / 12\end{array}$ & \begin{tabular}{|l|l|} 
Total LOS & postop LOS \\
4.3 days & 3.0 days \\
\end{tabular} \\
\hline 44 & \begin{tabular}{|l|} 
Discharge destination \\
\end{tabular} & KRU & \begin{tabular}{ll|l|l|l|}
$U$ & discharge : $25 / 7$ \\
\end{tabular} \\
\hline 45 & Dietitian referral & 1. Yes & 1024 \\
\hline
\end{tabular}

Data collection form - V Ward Feb 2012 - version 11 


\section{Appendix N}

Study data summary

\section{Demographics}

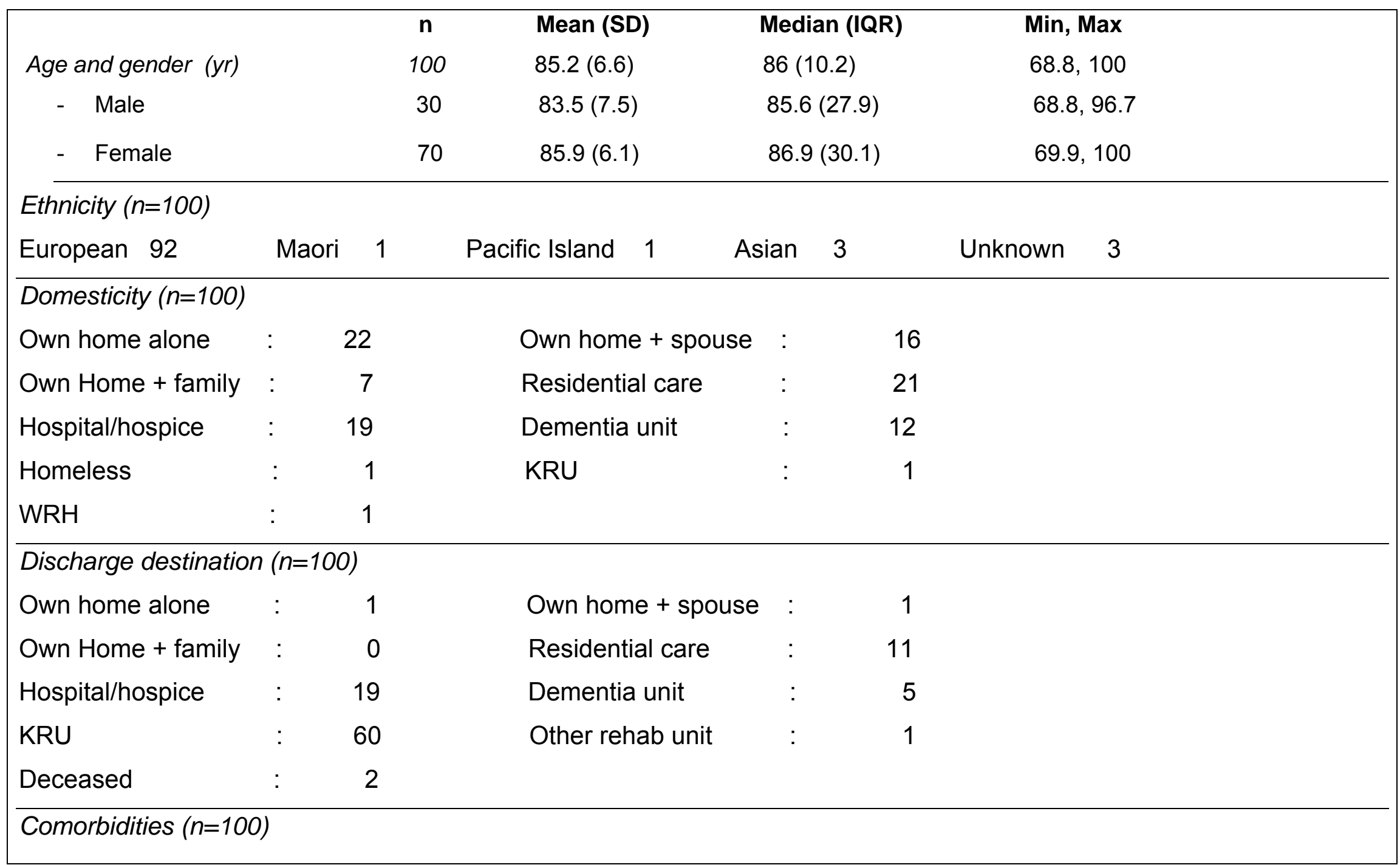




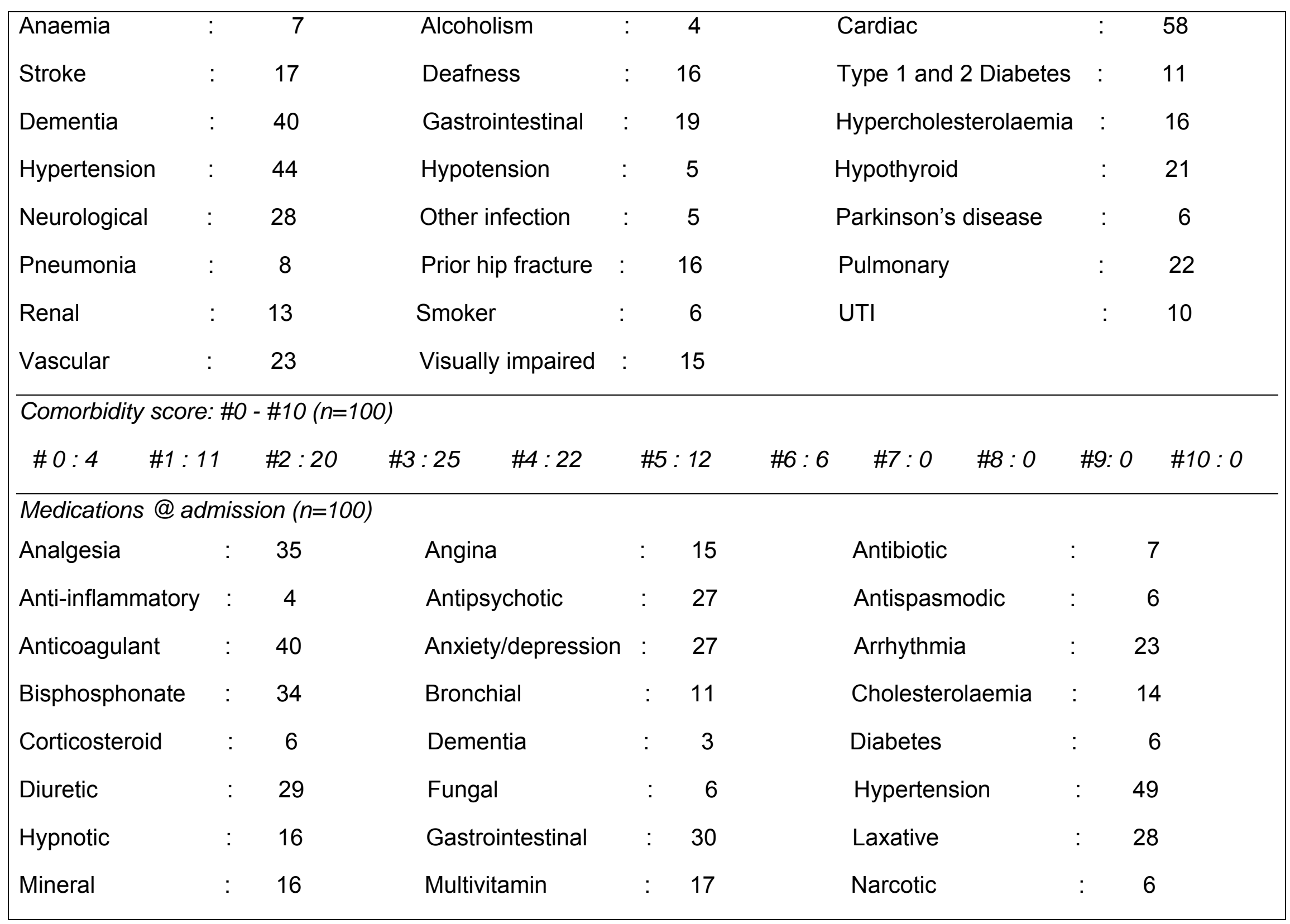




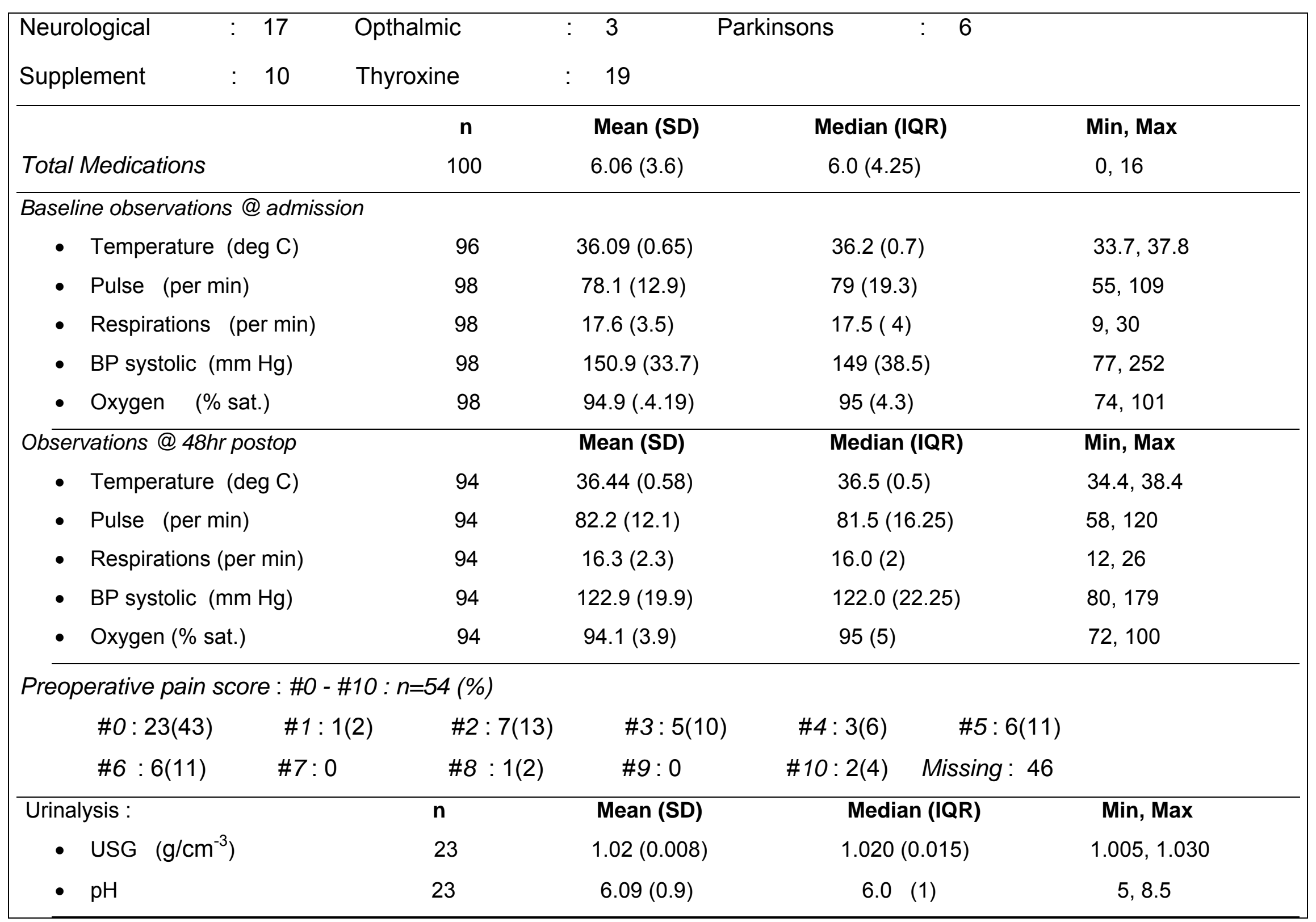




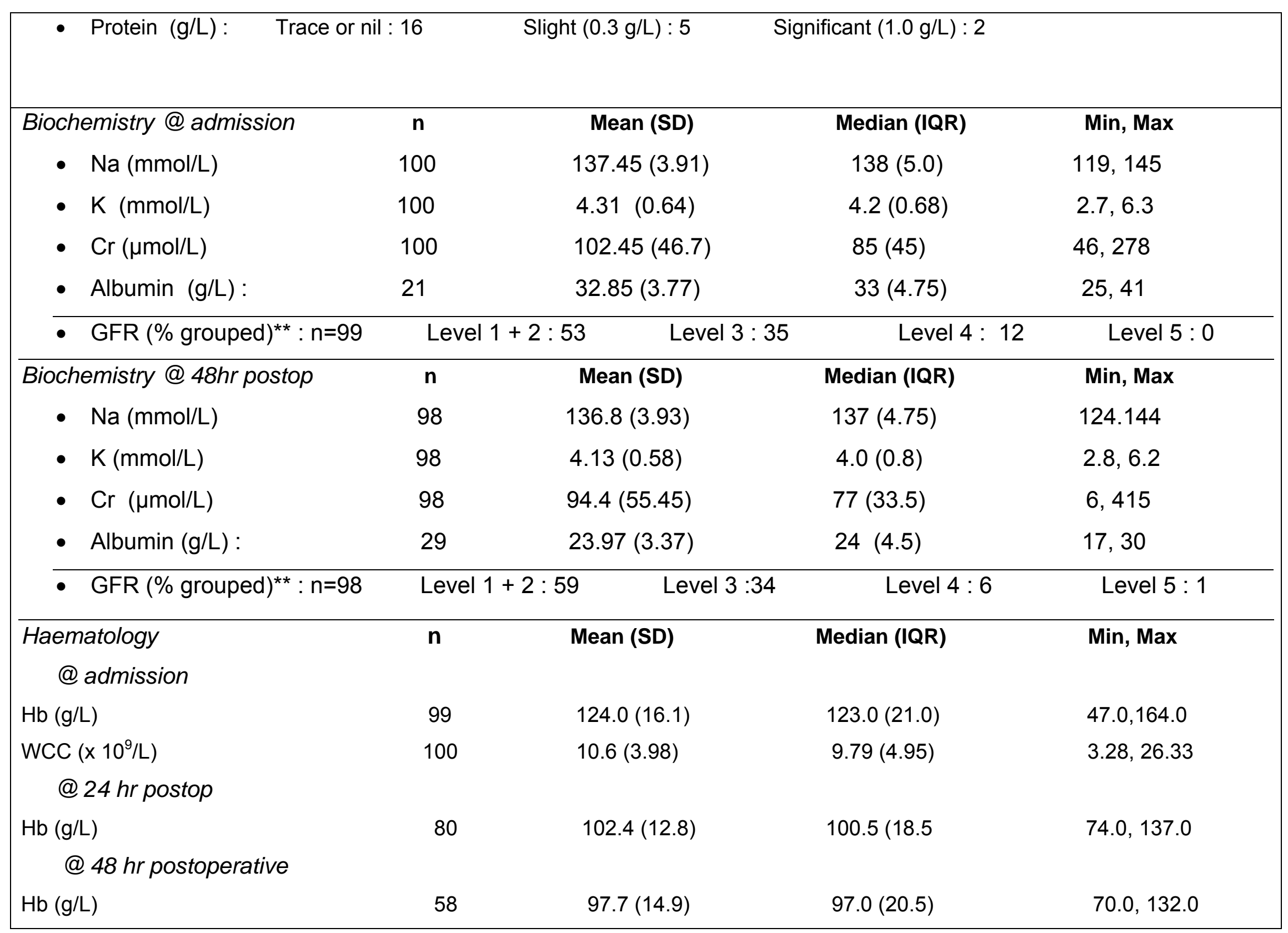




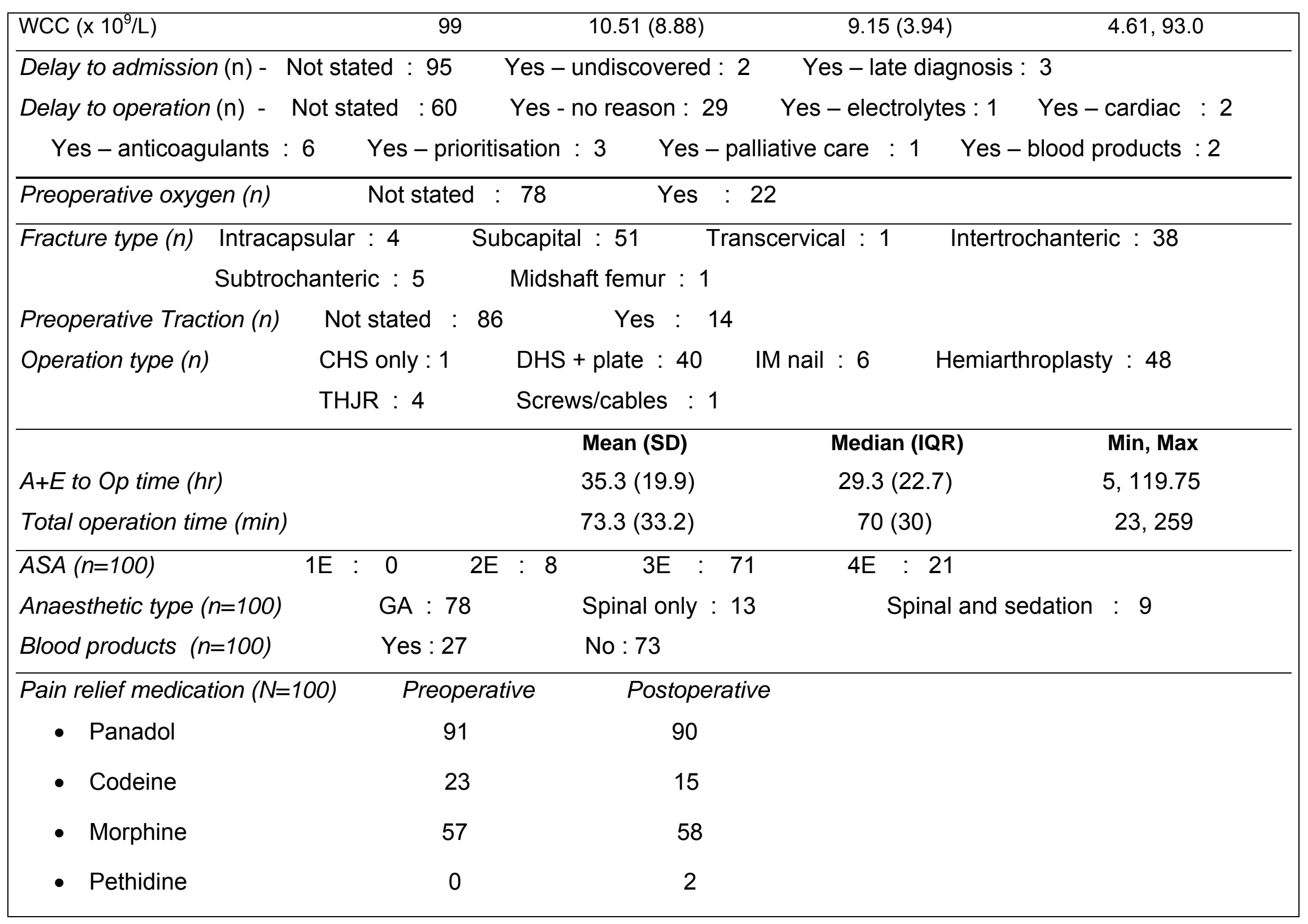




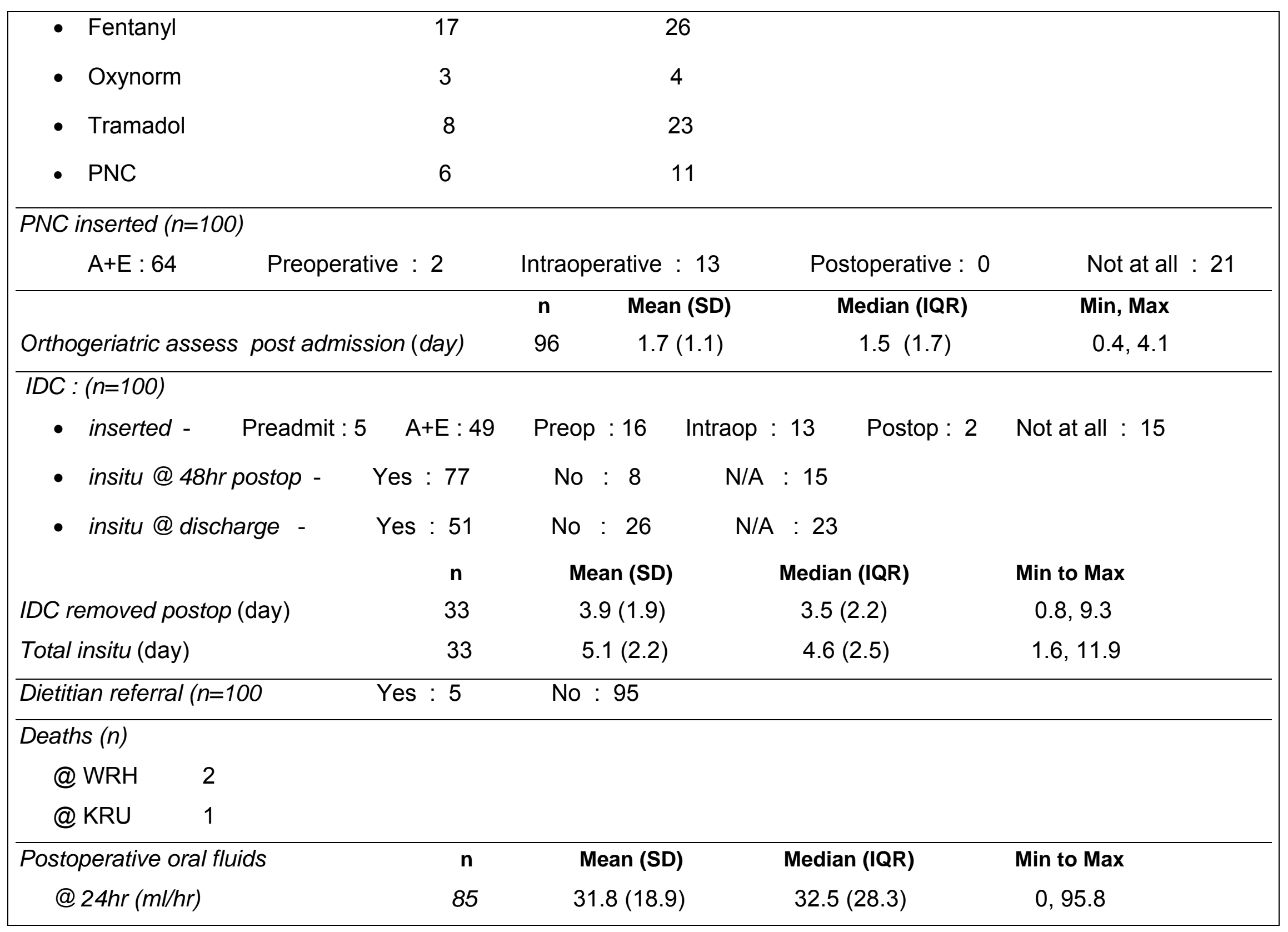




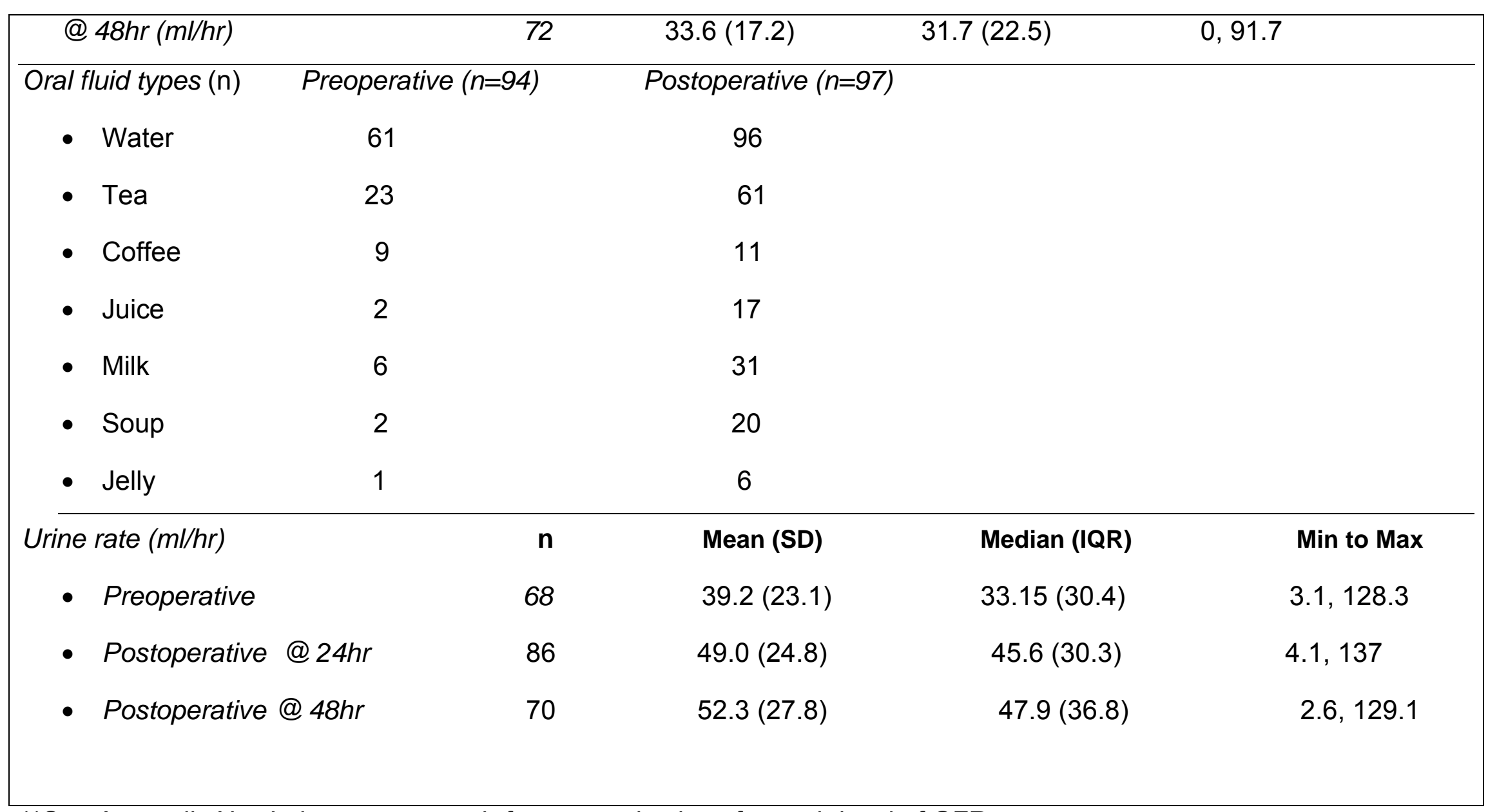

\footnotetext{
${ }^{* *}$ See Appendix $\mathrm{N}$ - Laboratory normals for grouped values for each level of GFR
} 


\section{Preoperative fluid management}

\begin{tabular}{|llllll|}
\hline & & $\mathbf{n}$ & Mean (SD) & Median (IQR) & Min to Max \\
i. & a. Preop oral fluids start (hr post admit) & 60 & $11.4(7.3)$ & $9.4(9.1)$ & $2.0,36.5$ \\
& b. Preop IV fluids start (hr post admit) & 89 & $9.6(7.6)$ & $7.3(7.9)$ & $0.3,39.0$ \\
$\quad$ c. Combined preop fluids start (hr post admit) & 90 & $8.2(6.9)$ & $6.0(6.2)$ & $0.3,39.0$ \\
ii. $\quad$ Preop oral fluid rate (ml/hr) & 58 & $11.7(8.5)$ & $10.5(12.1)$ & $0.8,39.7$ \\
\hline iii. $\quad$ Last preop fluids (hr preop) & 32 & $11.6(5.8)$ & $11.6(9.1)$ & $2.0,24.8$ \\
\hline
\end{tabular}




\section{Outcomes}

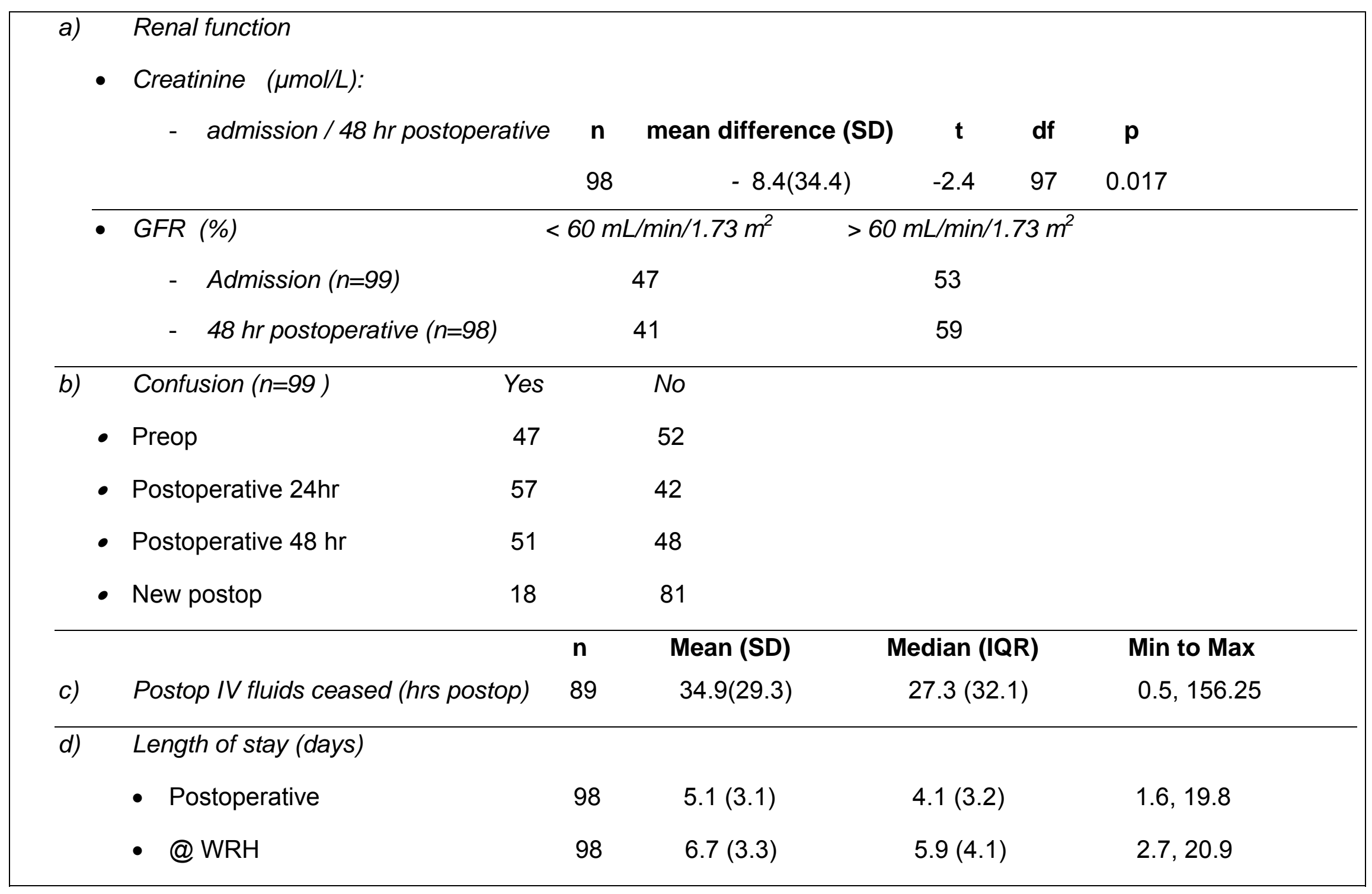




\begin{tabular}{llllll|}
\hline$\bullet @ \mathrm{KRU}$ & 57 & $21.4(12.9)$ & $19.9(15.9)$ & 3.071 .1 \\
$\bullet$ & CCDHB Total & 57 & $26.9(14.1)$ & $23.8(15.5)$ & $8,88.7$ \\
\hline
\end{tabular}

All data is subject to missing data as explained in Chapter 4 - Results 
Appendix $\mathrm{O}$

Laboratory normals

\section{Urinalysis and microbiology norms}

\begin{tabular}{|cl|}
\hline Indicator & Parameters \\
\hline Urinalysis & \\
- USG & $1.003-1.035 \mathrm{~g} / \mathrm{cm}^{-3}$ \\
- Protein & $<0.15 \mathrm{~g} / \mathrm{L}$ \\
- $\mathrm{pH}$ & $4.6-8(7=$ neutral $)$ \\
\hline Biochemistry & $115-160 \mathrm{~g} / \mathrm{L}$ \\
- $\mathrm{Hb}$ & $4.0-11.0 \times 10^{9} / \mathrm{L}$ \\
- $\mathrm{WCC}$ & $34-46 \mathrm{~g} / \mathrm{L}$ \\
- $\mathrm{Na}$ & $139-146 \mathrm{mmol} / \mathrm{L}$ \\
- K & $3.5-4.9 \mathrm{mmol} / \mathrm{L}$ \\
& $62-103 \mu \mathrm{mol} / \mathrm{L}$ \\
\hline
\end{tabular}

Biochemistry norms for healthy adult as per CCDHB laboratory reporting "Laboratory reports registered a "trace" of protein was recorded as zero (0), however this is indicative of protein content of up to $20 \mathrm{mg} / \mathrm{cm}^{-3}$.

\section{Glomerula filtration rate (GFR) *}

\begin{tabular}{|c|c|c|c|}
\hline Stage & Paramete & & Indication \\
\hline 1 & $>90 \mathrm{~mL} / \mathrm{m}$ & $1.73 \mathrm{~m}^{2}$ & Healthy kidney \\
\hline 2 & $60-89$ & “ & Mild kidney damage as per declining age \\
\hline 3 & $30-59$ “ & “ & Moderate kidney damage \\
\hline 4 & $15-29$ & “ & Severe kidney damage \\
\hline 5 & $<15$ & “ & Kidney failure requiring dialysis \\
\hline
\end{tabular}

*represented as stages of renal impairment

(Gwinnutt, 2008) 
Appendix $\mathrm{P}$

Fluid balance chart

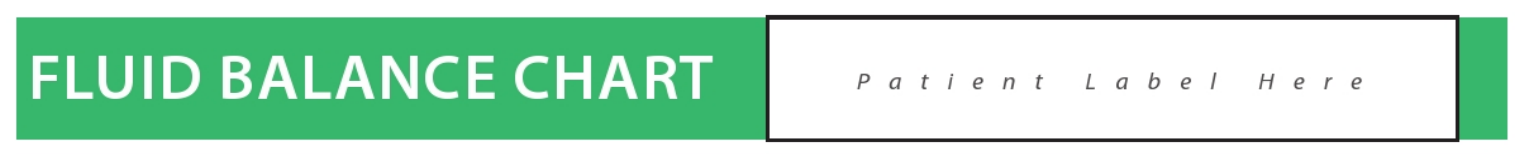

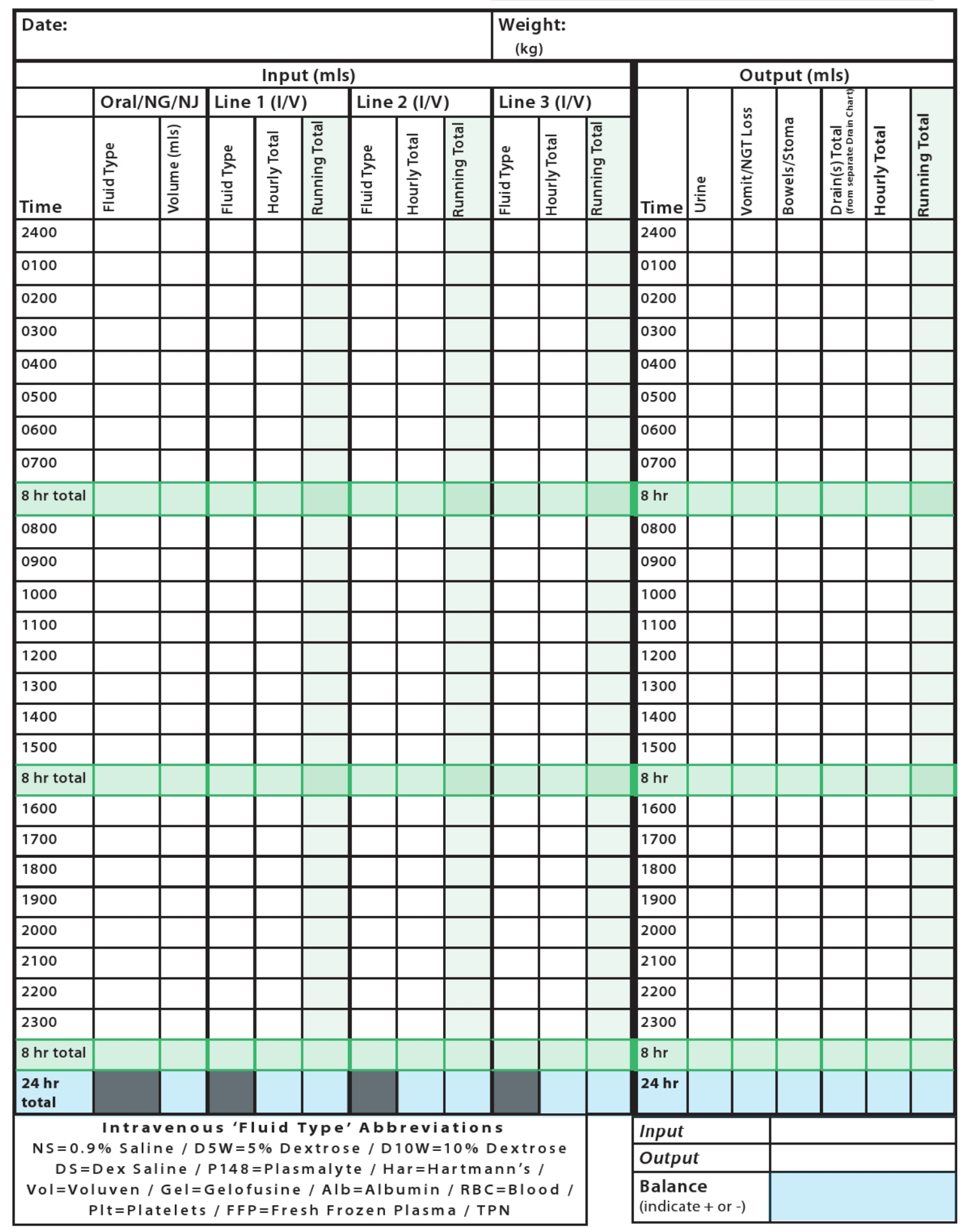


PREOPERATIVE FLUIDS

Appendix Q

General medicine - Fluid balance chart

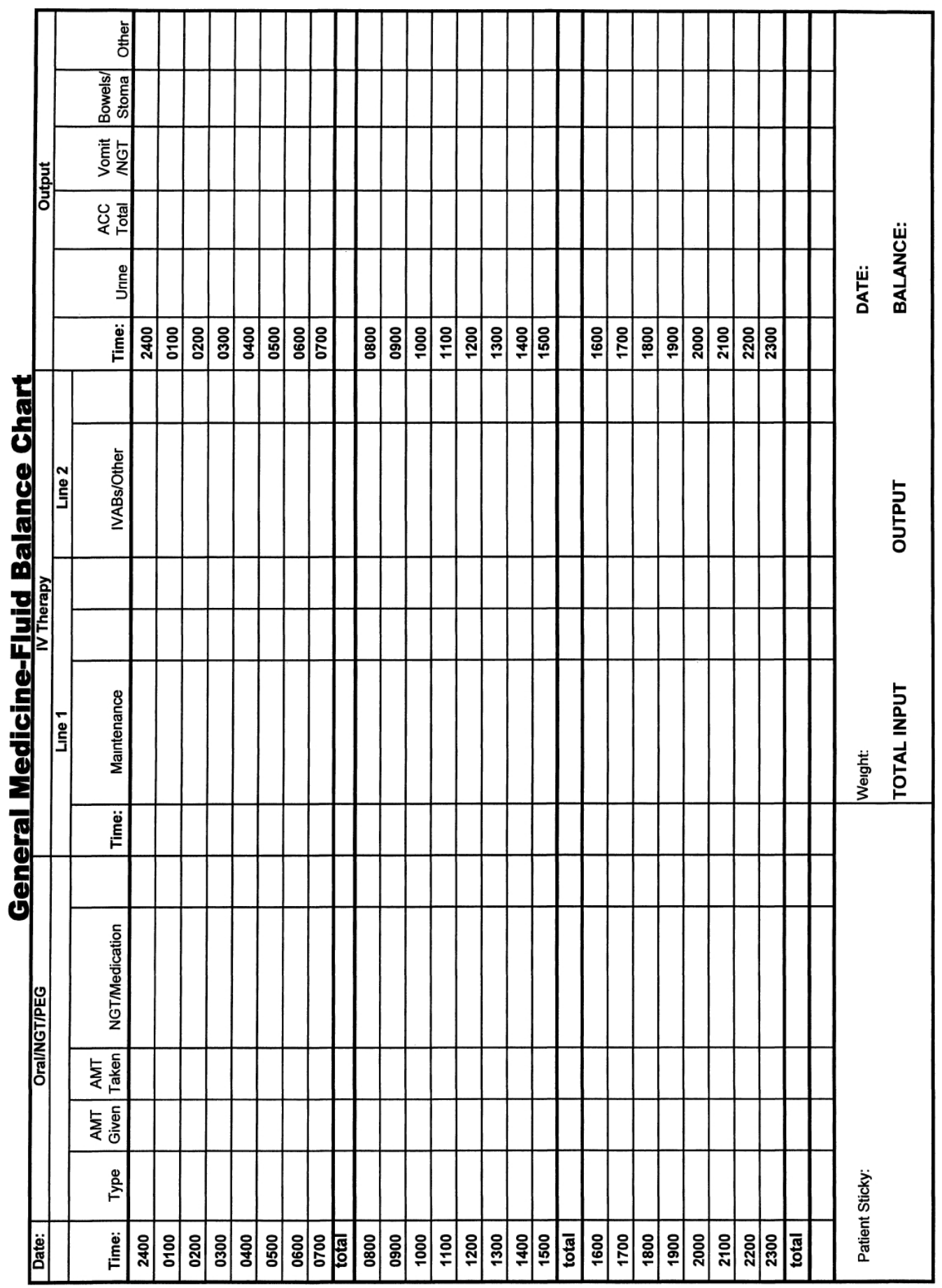


Appendix $\mathrm{R}$

Fluid balance chart - summary

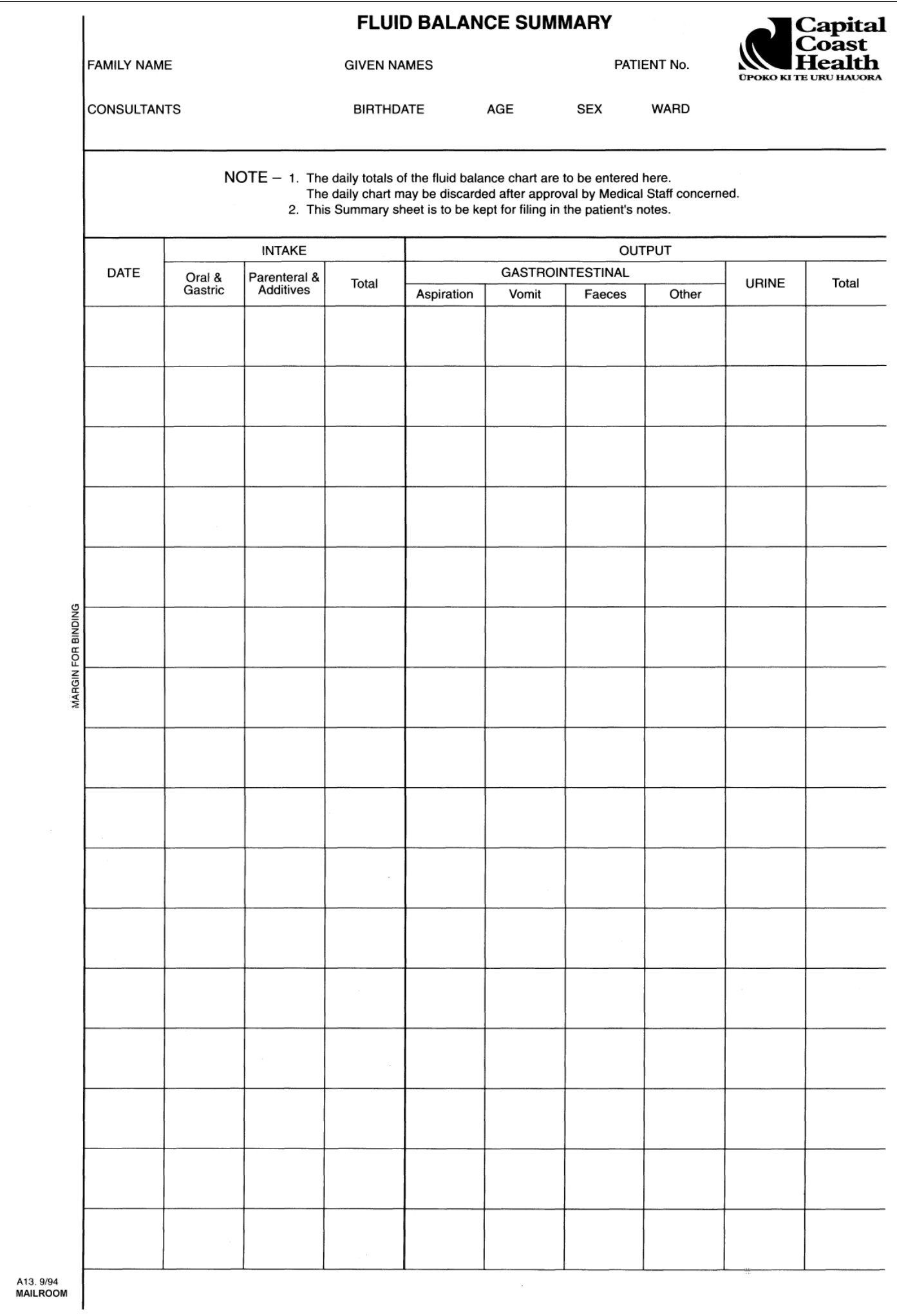




\section{Appendix S}

Confusion indicators

The following comments have been sourced from patient notes during the study and utilised as an indicator of confusion:

- Confused and agitated

- Not responding to voice

- Combative

- Incoherent verbalisation

- Unresponsive to communication

- Unable to follow more than one-step directions

- Not opening eyes to loud talking

- Doesn't know why she is in hospital

- Episodes of confusion

- Needs reorienting to time and place

- Very conversational although not orientated

- Agitated at times

- Alert but disorientated

- Confusion arises in the evening

- Restless and fidgety

- Not paying attention

- Agitated state

- Inappropriate conversation

- Seems confused

- Loud and disruptive

- Pleasantly confused

- Patient is demented and agitated during turns

- Became very confused in evenings

- Unable to get pain score as patient very agitated

- Patient very confused and paranoid that nurses are stealing her things 
- Random conversation and activities

- Pleasantly confused

- Alert and confused

- Some clear conversation

- Confused but comfy and not in pain

- Sometimes disorientated

The following statements, taken from patient notes during this study, are associated with those patients who appeared confused (as indicated by the above list) and further qualify the state of confusion:

- Lethargic

- Throwing pillows and blankets on the floor

- Requires watch

- Attempting to remove IDC

- Agitation could be a response to febrile condition

- Agitation could be a response to pain, possible sepsis, or Fentanyl patch

- Tugging at IV cannula

- Constantly trying to take off her clothes

- Patient paranoid about nurses taking her jewelry

- Responds to pain and voices at times

- My husband doesn't know I am here

The following statements, taken from patient notes during this study, were not used to indicate confusion:

- Easily reoriented

- Alert today

- Nil agitation or restlessness noted

- Disorientated at times but not confused

- Patient remains very drowsy 


\section{References}

Abou-Setta, A. M., Beaupre, L. A., Rashiq, S., Dryden, D. M., Hamm, M. P., Sadowski, C. A., et al. (2011). Comparative Effectiveness of Pain Management Interventions for Hip Fracture: A Systematic Review. Annals of Internal Medicine, 155(4), 234-U280. doi: 10.1059/0003-4819-155-4201108160-00346

Accident Compensation Corporation. (2005). Preventing injury from falls : the national strategy 2005-2015. Wellington: New Zealand ACC.

Adunsky, A., Arad, M., Blumstein, T., Weitzman, A., \& Mizrahi, E. (2008). Discharge hemoglobin and functional outcome of elderly hip fractured patients undergoing rehabilitation. European journal of physical and rehabilitation medicine, 44(4), 417-422.

Bennett, J., Thomas, V., \& Riegel, B. (2004). Unrecognized chronic dehydration in older adults: examining prevalence rate and risk factors. Journal of Gerontological Nursing, 30(11), 22-28.

Björkelund, K. B., Hommel, A., Thorngren, K., Lundberg, D., \& Larsson, S. (2011). Factors at admission associated with 4 months outcome in elderly patients with hip fracture. American Association of Nurse Anaesthetists Journal, 77(1), 49-58.

Bone and Joint Decade. (2012). Structure. Retrieved 13th June, 2012, from www.boneandjointdecade.org

Bossley, C., \& Miles, K. (2009). Musculoskeletal Conditions in New Zealand The Crippling Burden. Wellington: The Bone and Joint Decade 2000-2010.

Bottle, A., \& Aylin, P. (2006). Mortality Associated With Delay In Operation After Hip Fracture: Observational Study. British Medical Journal, 332(7547), 947-950.

Bowker, L., Price, C. C., \& Smith, S. (2006). Oxford Handbook of Geriatric Medicine. Oxford: Oxford University press. 
Brady, M., Kinn, S., Stuart, P., \& Ness, V. (2003). Preoperative fasting for adults to prevent perioperative complications. Cochrane Database of Systematic Reviews(4). doi: 10.1002/14651858.CD004423

Caccialanza, R., Klersy, C., Cereda, E., Cameletti, B., Bonoldi, A., Bonardi, C., et al. (2010). Nutritional parameters associated with prolonged hospital stay among ambulatory adult patients. Canadian Medical Association Journal, 182(17). doi: 10.1503/cmaj.091977

Capital and Coast District Health Board. (2007). Dementia guidelines MAN-27.

Wellington: Capital and Coast District Health Board.

Capital and Coast District Health Board. (2008). Clinical observations and monitoring of patients in medical and surgical nursing CPP AST-01. Wellington: Capital and Coast District Health Board.

Capital and Coast District Health Board. (2011). Perioperative Fasting of Adult Patients PER-05. Wellington: Capital and Coast District Health Board. Capital and Coast District Health Board Decision Support Unit (2012, ?? May ). [Hip fracture admission 2010/11].

Carson, J, P, C., \& Hebert, P. (2012). Transfusion thresholds and other strategies for guiding allogeneic red blood cell transfusion. Cochrane Database of Systematic Reviews(4). doi: 10.1002/14651858.CD002042.pub3

Connor, J., Langley, J., \& Cryer, C. (2006). International comparison of Injury deaths: Falls. New Zealand Injury Prevention Strategy Secretariat.

Davison, C., Merrilees, M., McKie, J., Sainsbury, R., \& Gilchrist, N. (2001). Hip fracture mortality and morbidity - can we do better? New Zealand Medical Journal, 114(1136), 329-332.

Dennison, E., Compston, J., Flahive, J., Siris, E., Gehlbach, S., Adachi, J., et al. (2012). Effect of co-morbidities on fracture risk: findings from the Global Longitudinal Study of Osteoporosis in Women (GLOW). Bone, 50(6), 1288-1293. doi: 10.1016/j.bone.2012.02.639

Department of Health and Human Services. (2011). A Profile of Older Americans. USA: Department of Health and Human Services. 
Duppils, G., \& Wikblad, K. (2000). Acute confusional states in patients undergoing hip surgery. Gerontology, 46, 36-43.

Faezah, S. K., Zhang, D., \& Yin, L. F. (2008). The prevalence and risk factors of delirium amongst the elderly in acute hospital. Singapore Nursing Journal, 35(1), 11-14.

Favus, M. (Ed.). (2006). Primer on the metabolic bone diseases and disorders of mineral metabolism (6th ed.). Washington: American Society for Bone and Mineral Research.

Fergus, L., Cutfield, G., \& Harris, R. (2011). Auckland City Hospital's orthogeriatric service:an audit of patients aged over 65 with fractured neck of femur. New Zealand Medical Journal, 124(1337).

Fick, D., Hodo, D., Lawrence, F., \& Inouye, S. (2007). Recognizing delirium superimposed on dementia. Journal of Gerontological Nursing, 33(2), 4047.

Foss, N. B., Jensen, P. S., \& Kehlet, H. (2007). Risk factors for insufficient perioperative oral nutrition after hip fracture surgery within a multi-modal rehabilitation programme. Age \& Ageing, 36(5), 538-543.

Foss, N. B., \& Kehlet, H. (2006). Hidden blood loss after surgery for hip fracture. Journal of bone and joint surgery (British), 88(8), 1053-1059. doi: 10.1302/0301-620X.88B8.17534

Gunnarsson, A., Lonn, K., \& Gunningberg, L. (2009). Does nutritional intervention for patients with hip fractures reduce postoperative complications and improve rehabilitation? Journal of Clinical Nursing, 18(9), 1325-1333. doi: 10.1111/j.1365-2702.2008.02673.x

Gwinnutt, C. (2008). Clinical Anaesthesia (3rd ed.). West Sussex: WileyBlackwell.

Handoll, H., Queally, J., \& Parker, M. (2011). Pre-operative traction for hip fractures in adults (Review). Cochrane Database of Systematic Reviews ( 12), Art. No.: CD000168. doi: 10.1002/14651858.CD000168.pub3.

Hanger, H., Smart, E., Merrilees, M., \& Frampton, C. (1999). The prevalence of malnutrition in elderly hip fracture patients. New Zealand Medical Journal, 112, 88-90. 
Hershkovitz, A., Kalandariov, Z., Hermush, V., Weiss, R., \& Brill, S. (2007).

Factors Affecting Short-Term Rehabilitation Outcomes of Disabled Elderly Patients With Proximal Hip Fracture. Archives of physical medicine and rehabilitation, 88(7), 916-921.

Juliebø, V., Bjøro, K., Krogseth, M., Skovlund, E., Ranhoff, A. H., \& Wyller, T. B. (2009). Risk factors for preoperative and postoperative delirium in elderly patients with hip fracture. Journal of the American Geriatrics Society, 57(8), 1354-1361. doi: 10.1111/j.1532-5415.2009.02377.x

Juliebø, V., Krogseth, M., Skovlund, E., Engedal, K., Ranhoff, A. H., \& Wyller, T. B. (2010). Delirium is not associated with mortality in elderly hip fracture patients. Dementia \& Geriatric Cognitive Disorders, 30(2), 112-120. doi: $10.1159 / 000318819$

Kagansky, N., Berner, Y., Koren-Morag, N., Perelman, L., Knobler, H., \& Levy, S. (2005). Poor nutritional habits are predictors of poor outcome in very old hospitalised patients. American journal of Clinical Nutrition, 82, 784-791.

Leung, A. H.-C., Lam, T.-P., Cheung, W.-H., Chan, T., Sze, P.-C., Lau, T., et al. (2011). An Orthogeriatric Collaborative Intervention Program for Fragility Fractures: A Retrospective Cohort Study. Journal of Trauma-Injury Infection and Critical Care, 71(5), 1390-1394. doi: 10.1097/TA.0b013e31821f7e60

Longmore, M., Wilkinson, I., Turmezei, T., \& Cheung, C. K. (2007). Oxford Handbook of Clinical Medicine (7th ed.). Oxford: Oxford University Press. Lundstrom, M., Edlund, A., Bucht, G., Karlsson, S., \& Gustafson, Y. (2003). Dementia after delirium in patients with femoral neck fractures. Journal of the American Geriatrics Society, 51(7), 1002-1006.

Marieb, E., \& Hoehn, K. (2007). Human Anatomy and Physiology (7th ed.). San Francisco: Pearson Benjamin Cummings.

Martini, F., \& Welch, K. (2006). A \& P Applications Manual. San Francisco: Pearson Education.

Mentes, J. (2006). A typology of oral hydration problems exhibited by frail nursing home residents. Journal of Gerontological Nursing, 32(1), 13-21. 
Mentes, J., \& Culp, K. (2003). Reducing hydration-linked events in nursing home residents. Clinical Nursing Research, 12(3), 210-225. doi:

$10.1177 / 1054773803252996$

Ministry of Health. (2002). Fracture of neck of femur services in New Zealand Hospitals 1999/2000. Wellington: Ministry of Health.

Ministry of Health. (2004). Guideline for specialist health services for older people. Wellington: Ministry of Health.

Ministry of Health. (2011). Targeting more elective operations. Wellington: Ministry of Health.

Miura, L. N., DiPiero, A. R., \& Homer, L. D. (2009). Effects of a geriatrician-led hip fracture program: improvements in clinical and economic outcomes. Journal of the American Geriatrics Society, 57(1), 159-167. doi: 10.1111/j.1532-5415.2008.02069.x

Montalvo, G., Pérez, G., Vega, M., Alarcón, A., de Linera, Á., Garay, G., et al. (2011). The acute orthogeriatric unit. Assessment of its effect on the clinical course of patients with hip fractures and an estimate of its financial impact. Spanish journal of geriatrics and gerontology, 46(4), 193-199.

National Clinical Guideline Centre. (2011). The management of hip fracture in adults. London: National Clinical Guideline Centre.

Nematy, M., Hickson, M., Brynes, A. E., Ruxton, C. H. S., \& Frost, G. S. (2006).

Vulnerable patients with a fractured neck of femur: nutritional status and support in hospital. Journal of Human Nutrition \& Dietetics, 19(3), 209218.

New Zealand Guidelines Group. (2003). Best practice evidence-based guideline : Acute management and immediate rehabilitation after hip fracture amongst people aged 65 years and over. Wellington: NZGG.

New Zealand Orthopaedic Association. (2003). Ageing of New Zealand.

Wellington: New Zealand Orthopaedic Association.

Nichol, C., Wilson, H., \& Webster, S. (2008). Elderly Care Medicine (7th ed.). Massachusetts: Blackwell.

Olsson, L., Karlsson, J., \& Ekman, I. (2007). Effects of nursing interventions within an integrated care pathway for patients with hip fracture. Journal of 
Advanced Nursing, 58(2), 116-125. doi: 10.1111/j.1365-

2648.2007.04209.x

Power, S., Kavanagh, D., McConnell, G., Cronin, K., Corish, K., Leonard, M., et al. (2012). Reducing preoperative fasting in elective adult surgical patients: a case-control study. Irish Journal of Medical Sciences, 181(1), 99-104

Price, J., Sear, J., \& Venn, R. (2004). Perioperative fluid volume optimization following proximal femoral fracture. Cochrane Database of Systematic Reviews(1). doi: 10.1002/14651858.CD003004.pub2

Rai, G., \& Mulley, G. (2007). Elderly Medicine - a training guide (2nd ed.). New York: Elsevier.

Roche, J. J. W., Wenn, R. T., Sahota, O., \& Moran, C. G. (2005). Effect Of Comorbidities And Postoperative Complications On Mortality After Hip Fracture In Elderly People: Prospective Observational Cohort Study. British Medical Journal, 331(7529), 1374-1376.

Royal College of Nursing. (2005). Perioperative fasting in adults and children. London: Royal College of Nursing.

Sainsbury, R., \& Richards, R. (1997). Prevention of osteoporosis inn older populations - a report to the National Health Committee. Wellington: National Advisory Committee on Health and Disability.

Sankoff, J., \& Zidulka, A. (2008). Non-invasive method for the rapid assessment of central venous pressure: Description and validation by a Single Examiner. Western Journal of Emergency Medicine, 9(4), 201-205.

Shiel, W., \& Stoppler, M. (Eds.). (2008). Webster's New World medical dictionary (3rd ed.). New Jersey: Wiley Publishing Inc.

Sieber, F. E., Mears, S., Lee, H., \& Gottschalk, A. (2011). Postoperative Opioid Consumption and Its Relationship to Cognitive Function in Older Adults with Hip Fracture. Journal of the American Geriatrics Society, 59(12), 2256-2262. doi: 10.1111/j.1532-5415.2011.03729.x

Spice, A. (2012, 27th Feb). [Hip fracture report 2010/11/12].

Statistics New Zealand. (2007). New Zealand's 65+ Population: A statistical volume. Wellington: Statistics New Zealand. 
Stoelting, R., \& Miller, R. (2007). Basics of anaesthesia (5th ed.). Philadelphia: Churchill Livingston.

Thwaites, J., Mann, F., Gilchrist, N., McKie, J., \& Sainsbury, R. (2007). Older patients with hip fractures: evaluation of a long-term specialist orthopaedic medicine service in their outcomes. New Zealand Medical Journal, 120(1254).

Wakefield, B., Mentes, J., Diggelmann, L., \& Culp, K. (2002). Monitoring Hydration Status in Elderly Veterans. Western Journal of Nursing Research, 24(132), 172-197. doi: 10.1177/01939450222045798

Ward, V. (2011). Impact of altered hydration status in elderly patients waiting for surgery: A review of the literature. The Dissector - Journal of the Perioperative Nurses College of the NZ Nurses Organisation, 39(3), 2934.

World Health Organisation. (2012). Dementia: a public health priority. Geneva: World Health Organisation.

Wotton, K., Crannitch, K., \& Munt, R. (2008). Prevalence, risk factors and strategies to prevent dehydration in older adults. Contemporary Nurse: $A$ Journal for the Australian Nursing Profession, 31, 44-56.

Young, W., Seigne, R., Bright, S., \& Gardner, M. (2006). Audit of morbidity and mortality following neck of femur fracture using the POSSUM scoring system. New Zealand Medical Journal, 119(1234).

Zeitz, K., \& McCutcheon, H. (2006). Observations and vital signs: ritual or vital for the monitoring of postoperative patients? Applied Nursing Research, 19(4), 204-211. doi: 10.1016/j.apnr.2005.09.005 